\author{
Universidade de São Paulo \\ Instituto de Física
}

\title{
Quantização de sistemas não-Lagrangianos e mecânica quântica não-comutativa
}

\author{
Vladislav Kupriyanov
}

Orientador: Prof. Dr. Dmitrii Maximovich Guitman

Tese de doutorado apresentada ao Instituto de Física para a obtenção do título de Doutor em Ciências

Comição Julgadora:

Prof. Dmitri Maximovich Guitman (IFUSP)

Prof. João Carlos Alves Barata (IFUSP)

Prof. Marcelo Otávio Caminha Gomes (IFUSP)

Prof. Francesco Toppan (CBPF)

Prof. Aleksandr Nikolaevich Pinzul (UnB)

São Paulo

2009 
FICHA CATALOGRÁFICA

Preparada pelo Serviço de Biblioteca e Informação do Instituto de Física da Universidade de São Paulo

\begin{tabular}{|c|}
\hline \multicolumn{1}{|c|}{ Kupriyanov, Vladislav } \\
\hline $\begin{array}{c}\text { Quantização de sistemas não-Lagrangianos e mecânica } \\
\text { quântica não-comutativa. São Paulo, 2009. }\end{array}$ \\
Tese (Doutorado) - Universidade de São Paulo. \\
Instituto de Física - Depto. de Física Nuclear
\end{tabular}




\section{Resumo}

Nesta tese apresentamos três problemas interligados: a quântização de teorias nãoLagrangianos, a mecânica quântica não-comutativa (MQNC) e a construção do produto estrela atravéz do ordenamento de Weyl. No contexto do primeiro problema foi elaborada uma abordagem da quantização canônica de sistemas com as equações de movimento não-Lagrangianas. Construímos um princípio da ação mínima para um sistema equivalente das equações diferenciais de primeira ordem. Existe uma ambiguidade não-trivial (que não se reduz a uma derivada total) na definição da função de Lagrange para os sistemas de equações de primeira ordem. Apresentamos uma descrição completa desta ambiguidade. O esquema proposto é aplicado para a quantização da teoria quadrática geral. Também foi construida a quantização do oscilador harmônico amortecido e da carga elétrica com radiação. No contexto da MQNC elaboramos uma formulação da integral de trajetória da MQNC relativística e construímos a generalização não-comutativa da ação da super-partícula. A quantização da ação proposta fornece as equações de Klein-Gordon e de Dirac nas teorias de campo não-comutativas. No contexto do terceiro problema desenvolvemos uma abordagem para a quantização por deformação no plano real com uma estrutura de Poisson arbitrária baseada no ordenamento simétrico dos produtos dos operadores. É formulado um procedimento iterativo simples e efetivo para a construção do produto estrela. Este procedimento nos permitiu calcular o produto estrela em ordens altas (em terceira e quarta ordens), algo que foi feito pela primeira vez. Exceto por uma análise da cohomologia, que não consideramos no artigo, o método proposto dá uma descrição explicita, na linguagem matemática usual da física, do produto estrela. ${ }^{1}$

\footnotetext{
${ }^{1}$ Autor agradece a Fundação de Amparo à Pesquisa do Estado de São Paulo (FAPESP) pelo apoio financeiro.
} 


\begin{abstract}
We present here three interrelated problems: quantization of non-Lagrangian theories, noncommutative quantum mechanics (NCQM) and a constructions of the star product trough the the Weyl ordering. In the context of the first problem an approach to the canonical quantization of systems with non-Lagrangian equations of motion is proposed. We construct an action principle for an equivalent first-order equations of motion. There exists an ambiguity (not reducible to a total time derivative) in associating a Lagrange function with the given set of equations. We give a complete description of this ambiguity. The proposed scheme is applied to quantization of a general quadratic theory. Also the quantization of a damped oscillator and a radiating point-like charge is constructed. In the context of NCQM we propose a path integral formulation of relativistic NCQM and construct a noncommutative generalization of superparticle action. After quantization, the proposed action reproduces the Klein-Gordon and Dirac equations in the noncommutative field theories. In the context of the third problem we develop an approach to the deformation quantization on the real plane with an arbitrary Poisson structure which based on Weyl symmetrically ordered operator products. A simple and effective iterative procedure of the construction of star products is formulated. This procedure allowed us to calculate the third and the fourth order star products. Modulo some cohomology issues which we do not consider here, the method gives an explicit and physics-friendly description of the star products.
\end{abstract}




\section{Sumário}

1 Introdução $\quad \mathbf{5}$

1.1 Quantização de sistemas não-Lagrangianos . . . . . . . . . . . . . . . . . . 6

1.2 Mecânica quântica não-comutativa . . . . . . . . . . . . . . . . . . . 11

1.3 Produto estrela através do ordenamento simétrico . . . . . . . . . . . . . . 12

1.4 Descrição dos capítulos . . . . . . . . . . . . . . . . . . . 13

2 Princípio de ação mínima para equações diferenciais $\quad 19$

2.1 Introdução . . . . . . . . . . . . . . . . . . . . . . . . . . . 19

2.1.1 Princípio de ação mínima para o sistema das equações da segunda ordem 20

2.1 .2 Considerações gerais . . . . . . . . . . . . . . . . . . 20

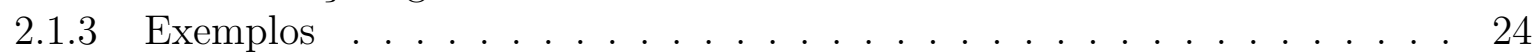

2.2 Princípio de ação mínima no formalismo de primeira ordem . . . . . . . . . . 25

3 Descrição Lagrangiana da carga elétrica com radiação 31

3.1 Introdução . . . . . . . . . . . . . . . . . . . . . 31

3.2 Impossibilidade de uma descrição Lagrangiana do sistema clássico de Lorentz-Dirac 32

3.3 O sistema das equações fisicamente equivalentes . . . . . . . . . . . . . . . . 34

3.3.1 Perturbativa redução de ordem na equação de Lorentz-Dirac . . . . . . . . 34

3.3 .2 A construção da Lagrangiana . . . . . . . . . . . . . . . . . . . . . . . 35

3.4 Funcional de ação no formalismo de primeira ordem . . . . . . . . . . . . . 37

4 Quantização canônica de sistemas não-Lagrangianos 38

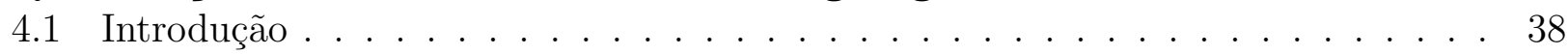

4.2 Formulação Hamiltoniana . . . . . . . . . . . . . . . . . . . . . 38

4.3 Quantização canônica . . . . . . . . . . . . . . . . . . . . . . . 40

4.4 Quantização de teorias quadráticas gerais . . . . . . . . . . . . . . . . . . 42

4.5 Quantização do oscilador harmônico amortecido . . . . . . . . . . . . . . . 44

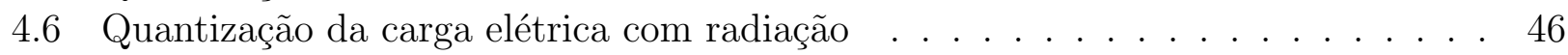

4.7 Conclusão . . . . . . . . . . . . . . . . . . . . . . . 48 
5 Integral de trajetória na MQNC $\quad 49$

5.1 Introdução . . . . . . . . . . . . . . . . . . . . . . . . 49

5.2 Representações via integral de trajetória dos propagadores da partícula em teoria quântica de campo não-comutativa . . . . . . . . . . . . . . . . . . 50

5.2 .1 O caso sem spin . . . . . . . . . . . . . . . . . 50

5.2 .2 Partícula spinorial . . . . . . . . . . . . . . . . 53

5.3 Ação pseudoclássica da partícula spinorial no espaço-tempo não-comutativo . . . 55

5.4 Integral de trajetória na mecânica quântica não-relativística no espaço não-

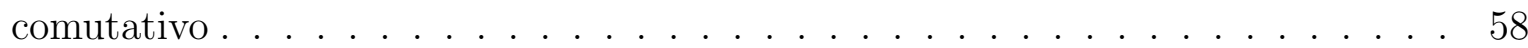

6 Produto estrela através do ordenamento simétrico $\quad 62$

6.1 Introdução . . . . . . . . . . . . . . . . . . . . . . . 62

6.2 Ordenamento de Weyl e produto estrela . . . . . . . . . . . . . . . . . 63

6.3 Propriedades gerais da expansão . . . . . . . . . . . . . . . . . . . . 65

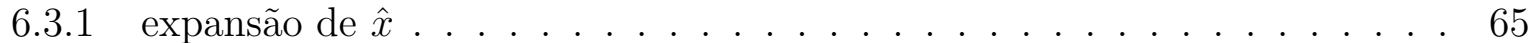

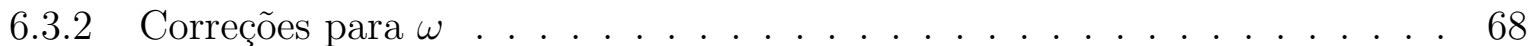

6.4 Calculação do produto estrela . . . . . . . . . . . . . . . . . . . . . . 69

6.4.1 Produto estrela de primeira ordem . . . . . . . . . . . . . . . . 69

6.4.2 Produto estrela de segunda ordem . . . . . . . . . . . . . . . . . . 70

6.4.3 Produto estrela de terceira ordem . . . . . . . . . . . . . . . . . . 72

6.4.4 Correções para o produto estrela de terceira ordem . . . . . . . . . . . 73

6.4.5 Produto estrela de quarta ordem . . . . . . . . . . . . . . . . 75

6.5 Conclusão . . . . . . . . . . . . . . . . . . . . . . 77

6.6 Algumas fórmulas . . . . . . . . . . . . . . . . . . . . . . . . . 77 


\section{Capítulo 1}

\section{Introdução}

Nesta tese, apresentamos três direções principais da pesquisa do autor durante seu doutoramento no IFUSP. São: a quantização de sistemas não-Lagrangianos, a mecânica quântica nãocomutativa (MQNC) e a construção do produto estrela através do ordenamento simétrico. O primeiro assunto trata do problema da construção do princípio da ação mínima e da quantização de sistemas físicos cuja dinâmica clássica é descrita por equações de movimento que não podem ser diretamente identificadas com as equações de Euler-Lagrange de alguma ação. Por isso estes sistemas se chamam sistemas não-Lagrangianos. Existem vários exemplos físicos de sistemas não-Lagrangianos e a quantização dessas teorias é um problema importante na física teórica.

O segundo tema da nossa pesquisa está ligado à mecânica quântica não-comutativa. Os argumentos gerais da mecânica quântica relativística indicam que não é possível medir o espaçotempo clássico na escala de Planck por causa dos efeitos do backreaction gravitacional. Isto levou à suposição de que em distâncias da ordem do comprimento de onda de Planck, o espaçotempo perde a sua estrutura de variedade contínua e suave e deve ser substituido por algum tipo de estrutura não-comutativa. A maioria dos artigos na área de MQNC trabalham com o caso do espaço não-comutativo plano, no qual os operadores das coordenadas $\hat{x}^{\alpha}$ têm relações de comutação: $\left[\hat{x}^{\alpha}, \hat{x}^{\beta}\right]=i \theta^{\alpha \beta}$, onde $\theta^{\alpha \beta}$ é uma matriz constante antisimétrica.

A terceira direção do nosso trabalho está ligada ao desenvolvimento de ferramentas matemáticas da geometria não-comutativa que possam ser usadas para o estudo de problemas conceituais da física na escala de Planck. Em particular, foi desenvolvido o método da construção perturbativa do produto estrela usando a representação polidiferencial da álgebra geral não-comutativa $\left[\hat{x}^{\alpha}, \hat{x}^{\beta}\right]=2 \varepsilon \omega_{P}^{\alpha \beta}(\hat{x})$, onde $\varepsilon$ é o parâmetro da deformação e $\omega_{P}^{\alpha \beta}(x)$ é uma estrutura de Poisson arbitrária.

Existe uma ligação entre os três assuntos mencionados. Como será mostrado na tese, a quantização canônica de lagrangianas de primeira ordem que aparecem no contexto de sistemas não-Lagrangianos levam a relações de comutação não triviais entre variáveis do espaço de fase,

$$
\left[\hat{x}^{\alpha}, \hat{x}^{\beta}\right]=i \omega_{N L}^{\alpha \beta}(\hat{x}),
$$


onde $\omega_{N L}^{\alpha \beta}=\Omega_{\alpha \beta}^{-1}$ e $\Omega_{\alpha \beta}$ é o multiplicador de integração para as equações não-Lagrangianas no formalismo de primeira ordem. No caso particular em que $\omega_{N L}^{\alpha \beta}$ é uma constante, ou seja, não depende de $\hat{x},(1.1)$ são exatamente as relações de comutação da MQNC. Assim, chegamos à conclusão de que formalmente a MQNC é um caso particular das teorias quânticas que correspondem aos sistemas não-Lagrangianos. Por outro lado, a matriz simpléctica $\omega_{N L}^{\alpha \beta}$ é um caso particular de estrutura de Poisson $\omega_{P}^{\alpha \beta}(x)$. Por isso, a representação polidiferencial dos operadores $\hat{x}^{\alpha}$, bem como o método da construção do produto estrela, são ferramentas matemáticas necessárias para a quantização de sistemas não-Lagrangianos e, em particular, para a MQNC.

Porém, historicamente cada um desses problemas se desenvolve independentemente dos outros. Por isso, escrevemos introduções separadas para cada um dos assuntos. Cada uma das introduções inclui uma revisão histórica, uma descrição dos problemas atuais na área e nossa contribuição na solução desses problemas. Na parte final da introdução, apresentamos a descrição dos capítulos que seguem.

\subsection{Quantização de sistemas não-Lagrangianos}

É bem conhecido que alguns sistemas físicos, por exemplo, a carga magnética, descrita primeiramente por Dirac [1], e os sistemas dissipativos [2,3], nos quais a interação com o ambiente é levada em conta por meio de uma força fenomenológica de atrito, são descritos geralmente em termos de equações diferenciais de segunda ordem que não podem ser identificadas diretamente com equações de Euler-Lagrange por um princípio de ação. No que segue, nós chamaremos tais equações de movimento de equações não-lagrangianas e os sistemas físicos correspondentes de sistemas não-Lagrangianos. A existência de um princípio de ação para um sistema físico dado ou, equivalentemente, a existência de uma função de Lagrange para tal sistema, permite que se realize a quantização canônica. Isto, em particular, enfatiza a importância de fornecer um princípio de ação para um sistema físico.

O problema da construção do princípio de ação mínima para equações de movimento dadas é conhecido na literatura como o problema inverso do cálculo de variações da mecânica Newtoniana. Este problema tem atraído um certo interesse em física por mais que um século. Ainda no ano de 1887, Helmholtz [4] propôs um critério para determinar se um sistema de equações diferenciais é um sistema lagrangiano ou não. Em 1894, Darboux [5] resolveu o problema inverso do cálculo de variações no caso unidimensional. O caso bidimensional foi investigado por Douglas [6] em 1941. Em particular, ele apresentou exemplos de equações diferenciais de segunda ordem que não podem ser obtidas do princípio de ação mínima (nenhuma lagrangiana existe para tais equações). Depois disso vários autores [7]-[13] investigaram o problema da construção de princípio variacional para sistemas multidimencionais.

Em 1974, Havas [14] propôs considerar o problema inverso do cálculo de variações no formalismo de primeira ordem.Para isso, é necessário passar as equações de movimento iniciais para um sistema equivalente de equações diferenciais da primeira ordem, introduzindo variáveis 
auxilares. Nos trabalhos [14]-[16], foi provado que no formalismo de primeira ordem o funcional da ação existe para qualquer sistema de equações diferenciais. Contudo, nos artigos que existem sobre este tema existem apenas teoremas de existência, já que nenhum deles responde à pergunta de como construír tal ação.

Também sabe-se que existe uma ambiguidade (que não se reduz a uma derivada total do tempo) na construção de uma função de Lagrange para as dadas equações diferenciais. No ano 1950 Wigner [17] propôs a seguinta pergunta: "Equações de movimento clássicas determinam as relações de comutação quânticas?" No próprio trabalho [17] Wigner assumiu que a resposta desta pergunta depende da forma da hamiltoniana, e em alguns sistemas pode ser negativa. Posteriormente, muitos autores (veja por exemplo [18]-[21]) investigaram este problema. Foi mostrado que a resposta à pergunda de Wigner é negativa, pois as relações de comutação quânticas dependem da ambiguidade na construção da lagrangiana para equações de movimento clássicas. Contudo, a descrição completa desta ambiguidade existe somente para caso unidimensional e foi dada ainda por Darboux [5] no final do século anterior. Existem tantas lagrangianas inequivalentes quanto funções de duas variáveis.

O problema da descrição quântica dos sistemas não-Lagrangianos, e, em particular, sistemas dissipativos, atrai atenção dos físicos há mais de 50 anos e têm várias aplicações importantes (veja por exemplo [2, 3], [22]-[45]). Historicamente, as primeiras tentativas de construír a teoria quântica dos sistemas dissipativos consistiram na busca de um método da quantização de equações de movimento que contêm a força de atrito proporcional a velocidade. O modelo mais popular e ao mesmo tempo mais símples é o oscilador harmônico amortecido [47] (damping oscillator),

$$
\ddot{x}+\alpha \dot{x}+\omega^{2} x=0,
$$

onde $\alpha$ - é o coeficiente fenomenológico de fricção e $\omega$ - frequência angular. Quando $\alpha \neq 0$, a equação (1.2) é uma equação não-lagrangiana. O fato da ausência da uma hamiltoniana torna impossível a quantização direta desse sistema. Portanto, primeiramente foi necessária a construção da formulação lagrangiana do oscilador amortecido.

Parece que a primeira tentativa de construír um funcional da ação para a equação (1.2) foi feita por Bateman [22] em 1931. Ele propôs a seguinte função de Lagrange

$$
L=y\left(\ddot{x}+\alpha \dot{x}+\omega^{2} x\right),
$$

onde $y$ - é uma nova variável. Obviamente a variação da ação correspondente pelo $y$ dará a equação inicial (1.2), mas a variação pelo $x$ dará mais uma equação

$$
\ddot{y}-\alpha \dot{y}+\omega^{2} y=0,
$$

que também descreve um oscilador harmônico mas com sinal oposto da força de atrito. Por isso ao contrário do primeiro oscilador amortecido, o segundo é um oscilador harmônico amplificado. Por causa disto, é conveniente pensar na variável y como uma variável efetiva que descreve um reservatório em que está contido o oscilador amortecido. Em seguida, este modelo recebeu 
o nome de modelo dual de Bateman [23]-[27]. Adicionando uma derivada total do tempo à Lagrangiana (1.3), esta transforma-se na Lagrangiana

$$
L=-\dot{x} \dot{y}+\frac{\alpha}{2}(x \dot{y}-y \dot{x})+\omega^{2} x y .
$$

Contudo, deve se notar que a Lagrangiana (1.3) bem como a Lagrangiana equivalente (1.4) descrevem um outro sistema dinâmico em relação à equação inicial (1.2). Particularmente, este sistema caracteriza-se pelo dobro de graus da liberdade e a hamiltoniana não é positivamente determinada. No nivel quântico, o modelo considerado não possui um espaço de Hilbert corretamente determinado [23]-[27].

Uma descrição hamiltoniana alternativa do oscilador harmônico amortecido foi proposta por Kanai [28] no ano 1948. Notou-se que a equação (1.2) torna-se uma equação Lagrangiana após a multiplicação pelo fator $e^{\alpha t}$. Este fator chama-se multiplicador de integração. A Hamiltoniana correspondente tem a forma:

$$
H=\frac{1}{2} e^{-\alpha t} p^{2}+\frac{1}{2} e^{\alpha t} \omega^{2} x^{2} .
$$

Obviamente, neste caso existe uma dependência explícita da hamiltoniana (1.5) no tempo. A quantização canônica deste modelo foi construída nos trabalhos [28]-[31]. Notamos contudo que o método presente da construção da formulação Hamiltoniana não pode ser aplicado a qualquer sistema não-Lagrangiano, pois o multiplicador de integração não existe não todo sistema de equações diferenciais. Os exemplos correspondentes, bem como a teoria geral do multiplicador de integração, serão considerados nos artigos aplicados.

Finalmente, existe mais uma abordagem à quantização dos sistemas dissipativos que trata a dissipação como o resultado da interação de um sistema mecânico $S$ com algum reservatório térmico $E$ (veja [32]-[41]). Já que o sistema completo $S+E$ é um sistema fechado e, por isso, hamiltoniano, ele permite que se realize a quantização canônica. Posteriormente, para obter a matriz densidade $\rho_{S}$ que descreve o sistema dissipativo $S$ é necessário calcular a média (integração) sobre os estados do reservatório $E$.

Esta abordagem também tem deficiências porque ela é bastante complicada e necessariamente precisa da fixação de um modelo do reservatório, por isso o resultado da quantização depende dos parâmetros do modelo escolhido. No caso mais simples, quando o reservatório é modulado como um conjunto infinito de osciladores harmônicos, a resposta final depende de parâmetros do modelo tais como temperatura $T$ e frequências próprias dos osciladores $\omega_{k}$. Além disso, esta abordagem pode ser aplicada somente para a quantização de sistemas dissipativos, quando a causa do carácter não-Lagrangiano das equações de movimento é a dissipação, ou seja, a interação com o ambiente. Caso o sistema considerado seja um sistema não-Lagrangiano por outra causa, por exemplo o monopólo magnético, esta abordagem perde qualquer sentido.

Assim, todas as abordagems à quantização dos sistemas não-Lagrangianos mencionadas têm deficiências. Contudo, algumas questões ligadas à quantização, em particular dos sistemas dissipativos, estão em aberto até agora. Assim, por exemplo, é interessante a análise da influência da dissipação em fenômenos quânticos tais como tunelamento e interferência quântica. Um 
aparecimento possível de tunelamento quântico em nível macroscópico em criogênese foi descrito por [42]. A influência da dissipação no tunelamento quântico em sistemas macroscópicos foi considerada pela primeira vez nos trabalhos [43]-[45], onde se mostrou que a dissipação multiplica a probabilidade de tunelamento por um fator que depende do coeficiente fenomenógico de atrito. A interferência quântica na presença de dissipação foi investigada pelos mesmos autores no trabalho [46]. Não obstante, a fim de descrever a dissipação, os autores de [43]-[46] usam uma abordagem que trata o atrito como uma interação do sistema com algum reservatório em que está contido. As deficiências desta abordagem já foram discutidas acima.

Existem outros problemas físicos interessantes ligados à quantização dos sistemas nãolagrangianas. Notamos apenas dois deles: (i) a descrição quântica de efeitos de backreaction de radiação (radiation backreaction), que é baseada na quantização de equações não-lagrangianas que descrevem a dinâmica efetiva de objetos carregados (como partículas, cordas, etc.) em campos externos com a backreaction de radiação e (ii) a quantização da carga magnética, primeiramente construída por Dirac [1]. Na teoria [1], o problema da natureza não-lagrangiana das equações de movimento para uma carga magnética no campo elétrico foi resolvido usando um potencial singular que conduz a termos não-físicos do tipo $\delta$ na força de Lorentz. E com o fim de eliminar estes termos, o então chamado "veto de Dirac" foi imposto para proibir o elétron de ser encontrado na trajetória do monopólo. Fora da trajetória, as equações de movimento que resultam do princípio da ação coincidem com as iniciais. Note, entretanto, que a teoria de Dirac não está livre de críticas [48].

Assim, chegamos à conclusão de que há necessidade do desenvolvimento de novas abordagems para a quantização de sistemas físicos a partir de equações de movimento, aplicáveis a qualquer sistema de equações diferenciais e não envolverão construções auxilares como reservatório térmico ou "veto de Dirac". A partir disso, foram formuladas as metas do nosso trabalho:

- O desenvolvimento de métodos para a solução do problema inverso do cálculo das variações para um sistema dinâmico de forma geral.

- O desenvolvimento de métodos de quantização de sistemas físicos a partir das equações de movimento e aplicação destes métodos à quantização de sistemas não-Lagrangianos.

Neste caminho foram alcançados os seguintes resultados principais:

1. Elaborado um novo método efetivo para a construção do princípio variacional para um sistema físico partindo das equações de movimento. Caso um sistema de equações de movimento não admita a existência de princípio de ação mínima, este sistema sempre pode ser reformulado como um sistema equivalente de equações Lagrangianas de primeira ordem. construímos explicitamente a ação Lagrangiana que leva a tal sistema bem como apresentamos uma descrição completa da ambiguidade na definição desta ação. 
2. Elaborada uma abordagem para a quantização canônica de teorias não-lagrangianas baseada na formulação Lagrangiana proposta de um sistema equivalente à equações da primeira ordem. Foi construída a quantização canônica de teorias quadráticas gerais (sistemas com as equações de movimento lineares). Provado que a evolução quântica dos sistemas considerados é completamente determinada pela evolução clássica correspondente.

3. Foi construída a quantização de exemplos importantes de sistemas dissipativos: oscilador harmônico amortecido e carga elétrica com radiação (radiating point-like charge). Obtivemos expressões exatas para a evolução dos valores médios da energia nos exemplos considerados.

4. Obtidos pela primeira vez:

a A forma explícita da Lagrangiana de sistemas de equações diferenciais de segunda ordem que admitem a existência de um multiplicador de integração, isto é, uma matriz multiplicadora que pode dotar um sistema de equações diferenciais com a estrutura de equações de Euler-Lagrange. A partir de considerações gerais, obtivemos condições necessárias e suficientes para a existência do multiplicador de integração para equações diferenciais. $\mathrm{O}$ método proposto para a solução do problema inverso do cálculo de variações é ilustrado por diferentes exemplos. Foi construída a ação Lagrangiana de sistemas dissipativos multidimensionais no formalismo de segunda ordem. Foi também considerado um exemplo de sistema dinâmico linear com equações de movimento que não admitem a existência de um multiplicador de integração.

b Um procedimento explícito para a construção do funcional de ação para os sistemas de equações diferenciais de primeira ordem usando a solução do problema de Cauchy dos sistemas considerados. Obtivemos a descrição completa da ambiguidade não trivial (que não pode ser reduzida à adição de uma derivada total em relação ao tempo) na definição da função de Lagrange para os sistemas de equações de primeira ordem. Foi construída uma ação quadrática para as teorias com equações do movimento lineares não-homogêneas arbitrárias (sistemas dinâmicos lineares).

c A prova da inexistência do princípio de ação mínima para a equação relativística de Lorentz-Dirac bem como para o seu limite não relativístico. É proposta uma equação reduzida de Lorentz-Dirac que descreve a dinâmica efetiva da carga elétrica com radiação e não tem soluções não-físicas. Para esta equação, o problema inverso do cálculo de variações pode ter várias soluções. Como um exemplo, é considerada uma carga pontual não relativística no campo magnético constante e homogêneo com radiação. Mostra-se que este sistema admite a existência do funcional de ação no formalismo de segunda ordem mas nenhuma das lagrangianas possíveis transforma-se, no limite do desligamento da interação $e \rightarrow 0$, na Lagrangiana usual da partícula livre $L=m \dot{\mathbf{x}} / 2$. Uma descrição 
física satisfatória para este sistem foi obtida com um sistema equivalente de equações diferenciais de primeira ordem.

d Uma abordagem baseada na formulação Lagrangiana proposta no formalismo de primeira ordem para a quantização canônica de teorias não-Lagrangianas. A hamiltonização da teoria Lagrangiana construída conduz a uma teoria hamiltoniana com vínculos dependentes do tempo. A quantização canônica de tal teoria é um problema não trivial (isto segue das considerações gerais presentes no livro [49] de Gitman e Tyutin).Adotamos a abordagem geral de hamiltonização e de quantização canônica para teorias com vínculos dependentes do tempo para este caso de teorias não-lagrangianas. O esquema da quantização proposta foi ilustrado no exemplo de sistemas dinâmicos lineares.

\subsection{Mecânica quântica não-comutativa}

Teorias não-comutativas constituem um ramo da física teórica moderna em rápido desenvolvimento [50]. A primeira vez em que coordenadas não-comutativas surgiram no contexto de teoria quântica de campos foi nos trabalhos pioneiros de Heisenberg e Snyder há mais de 50 anos. Posteriormente, a não-comutatividade foi estudada na abordagem de quantização por deformação [51], que relaciona quantização com deformação das estruturas de Poisson. Em trabalhos mais recentes [52] foi mostrado que o limite de baixa energia da teoria $M$, bem como das teorias de supercordas, levam diretamente às teorias de calibre não-comutativas.

É bem sabido também que ao se considerar o problema de Landau (uma partícula carregada sujeita a se mover num plano na presença de campos magnéticos perpendiculares constantes), chega-se a coordenadas não-comutativas. Este fato foi utilizado em [53] para sugerir uma abordagem do efeito Hall quântico baseado em geometria não-comutativa.

Outra motivação atual para o estudo das teorias não-comutativas se relaciona à idéia de que ela poderia ser uma formulação matemática razoavelmente simples para representar a proposta de Wheeler e de Witt de spacetime foam, i.e., da idéia de que na ordem do comprimento de Planck $\left(10^{-33} \mathrm{~cm}\right)$, na qual os efeitos da gravitação se tornam extremamente fortes, o espaço-tempo perde sua estrutura contínua e deve envolver flutuações quânticas de geometria e topologia [54].

Uma área particular das teorias não-comutativas é a mecânica quântica não-comutativa (MQNC), que recentemente tem ganho bastante atenção [55]-[67]. No caso mais simples, o espaço-tempo não-comutativo pode ser realizado pelos operadores de coordenada $\hat{x}^{\alpha}$ com as seguintes relações de comutação

$$
\left[\hat{x}^{\alpha}, \hat{x}^{\beta}\right]=i \theta^{\alpha \beta}
$$

em que $\theta^{\alpha \beta}$ é uma matriz antissimétrica constante a valores reais. Como foi mencionado na introdução geral a MQNC é um caso particular das teorias quânticas que correspondem a sistemas não lagrangianos. Por um lado, isto dá origem a uma grande classe de exemplos naturais de sistemas quânticos não-comutativos. Por outro lado, podemos usar as ferramentas 
da MQNC [55]-[67] para examinar a natureza quântica de sistemas não-Lagrangianos e viceversa.

Deve-se notar que as ações clássicas de teorias de campo no espaço-tempo não-comutativo podem ser escritas como ações clássicas modificadas mas já no espaço-tempo comutativo (introduzindo o produto da estrela). Depois da quantização de tais ações, que chamamos de ações $\theta$-modificadas, os efeitos da não-comutatividade do espaço-tempo se manisfestarão através de características da teoria quânticas de campo correspondente. Considerando a mecânica quântica de uma partícula (ou o sistema de $N$ partículas) com coordenadas não-comutativas, perguntase como construír a ação clássica $\theta$-modificada para este sistema de dimensão finita. Como no caso da teoria de campos, tal ação $\theta$-modificada deve, depois da quantização, manifestar os efeitos da não comutatividade do espaço-tempo através de características do sistema quântico correspondente. Neste caso, através da ação clássica recuperamos as relações não comutativas das coordenadas do espaço-tempo. Esta se tornou mais uma meta do nosso trabalho:

- A construção de ações $\theta$-modificaoas na mecânica quântica.

Os resultados principais são:

Elaborado um novo método para a construção da ação $\theta$-modificada na mecânica quântica relativística e não relativística. Foram construídas tais ações para partículas relativísticas com e sem spin. A idéia chave é extrair ações $\theta$-modificadas para partículas relativísticas da representação dos propagadores da teoria do campo não-comutativa correspondente via integral de trajetória. Consideramos equações de Klein-Gordon e de Dirac para propagadores causais em tais teorias. Posteriormente, construímos a representação destes propagadores via integral de trajetória. Tratamos as ações efetivas nesta representação como ações de partículas relativísticas $\theta$-modificadas. Para confirmar a interpretação, construímos a quantização canônica das ações obtidas, obtendo assim as equações de Klein-Gordon e de Dirac na teoria de campo nãocomutativa. A ação $\theta$-modificada da partícula relativística com spin é uma generalização da ação pseudoclássica da superpartícula de Berezin-Marinov [68] para o caso não-comutativo.

\subsection{Produto estrela através do ordenamento simétrico}

A história recente da quantização por deformação começou com o artigo [51], veja [69] para a revisão. Um ingrediente importante no programa da quantização por deformação é o produto estrela. Toma-se uma álgebra $\mathcal{A}$ das funções lisas na variedade de Poisson equipada com a estrutura de Poisson $\omega$ e deforma-se $\mathcal{A}$ em $\mathcal{A}_{\omega}$ introduzindo o produto estrela de tal forma que o comutador estrela de duas funções quaisquer resulte no parêntese de Poisson entre essas funções na ordem mais baixa da expansão em $\omega$. No ano de 1997 Kontsevich [70] demonstrou a existência do produto estrela em uma variedade de Poisson $C^{\infty}$ qualquer e apresentou uma fórmula que, em princípio, permite calcular este produto. Contudo, caso seja preciso o produto estrela em segunda ordem da expansão, a fórmula de Kontsevich não é muito útil, já que não 
existe um método sistemático do cálculo das integrais envolvidas. Além do mais, existe outra razão, de natureza psicológica, para procurar uma formulação mais simples do produto estrela. O maquinário do Formality Theorem é pesado demais para ser facilmente digerido por uma parte considerável da comunidade de física. Notamos também que o Formality Theorem na verdade fornece muito mais que somente o produto estrela. Portanto, já que a parte mais pesada do trabalho já foi feita, podemos começar a procurar por uma formulação do produto estrela em uma linguagem mais física que admita um algoritmo computacional mais simples para ordens mais altas da expansão. Nesa direção, nossa meta principal foi

- A elaboração de métodos efetivos para a construção perturbativa do produto estrela no caso geral.

Foram obtidos os seguintes resultados principais:

Foi desenvolvida uma nova abordagem para a quantização por deformação no espaço plano real com uma estrutura de Poisson $\omega^{\alpha \beta}(x)$ arbitrária baseada no ordenamento simétrico dos operadores $\hat{x}^{\alpha}$, que representam coordenadas cartesianas deformadas no $\mathbb{R}^{N}$. Nós requeremos que $\hat{x}^{\alpha}$ satisfaçam as relações de comutação gerais $\left[\hat{x}^{\alpha}, \hat{x}^{\beta}\right]=2 \varepsilon \omega^{\alpha \beta}(\hat{x})$ (onde $\varepsilon$ é o parâmetro da deformação) e então construímos a representação polidiferencial para $\hat{x}^{\alpha}$. Uma grande vantagem da nossa abordagem é a existência de um procedimento iterativo simples e efetivo em $\alpha$, tal que a ordem $(n-1)$ de $\omega^{\alpha \beta}(\hat{x})$ permita calcular a ordem $n$ de $\hat{x}^{\alpha}$, que por sua vez já determine a ordem $n$ de $\omega^{\alpha \beta}(\hat{x})$, etc. O uso desta abordagem nos permitiu calcular a expansão do produto estrela em terceira e quarta ordens, algo que foi feito pela primeira vez. Além disso, operamos com objetos bem conhecidos da mecânica quântica.

\subsection{Descrição dos capítulos}

No capítulo 2, consideramos a questão da construção do princípio da ação mínima para um dado sistema de equações diferenciais, usando o método do multiplicador de integração, isto é, uma matriz multiplicadora que pode dotar um sistema de equações diferenciais com a estrutura das equações de Euler-Lagrange. Na seção 2.2, apresentamos um método simples de construção de condições necessárias e suficientes para a existência do multiplicador de integração para equações de segunda ordem. Caso o multiplicador de integração exista e seja conhecido, apresentamos uma forma explícita da Lagrangiana. Na seção 2.2.2, é ilustrado, por diferentes exemplos, o método proposto para a solução do problema inverso do cálculo de variações. Foi construída a ação Lagrangiana de sistemas dissipativos multidimensionais no formalismo de segunda ordem. Também foi considerado o exemplo de um sistema dinâmico linear com equações de movimento que não admitem a existência do multiplicador de integração.

Por meio da introdução de $n$ variaveis auxiliares (por exemplo $p_{i}=\dot{q}^{i}, i=1, . ., n$ ) um sistema de $n$ equações não-Lagrangianas sempre pode ser apresentado na forma de um sistema 
equivalente de $2 n$ equações diferenciais de primeira ordem

$$
\begin{aligned}
\dot{x}^{\alpha} & =f^{\alpha}(t, x), \\
x^{\alpha} & =\left(q^{i}, p_{i}\right), \alpha=1, . ., 2 n .
\end{aligned}
$$

Na seção 2.3, mostramos que o multiplicador de integração $\Omega$ para o sistema de equações (1.6) deve satisfazer as condições seguintes: antissimetria

$$
\Omega_{\alpha \beta}=-\Omega_{\beta \alpha},
$$

identidade de Jacobi

$$
\partial_{\alpha} \Omega_{\beta \gamma}+\partial_{\beta} \Omega_{\gamma \alpha}+\partial_{\gamma} \Omega_{\alpha \beta}=0
$$

e a equação

$$
\partial_{t} \Omega_{\alpha \beta}+£_{f} \Omega_{\alpha \beta}=0,
$$

onde $£_{f} \Omega_{\alpha \beta}$ é a derivada de Lie da forma simplectica $\Omega_{\alpha \beta}$ ao longo do campo vetorial $f^{\gamma}$. Neste caso o multiplicador de integração $\Omega$ sempre existe e pode ser construído pela solução de Cauchi do sistema de equações de primeira ordem (1.6). A Lagrangiana correspondente tem a forma

$$
L=J_{\alpha} \dot{x}^{\alpha}-H
$$

onde

$$
\begin{gathered}
J_{\alpha}(t, x)=\int_{0}^{1} x^{\beta} \Omega_{\beta \alpha}(t, s x) s d s+\partial_{\alpha} \varphi(t, x), \\
H(t, x)=\int_{0}^{1} d s x^{\beta}\left[\Omega_{\beta \alpha}(t, s x) f^{\alpha}(t, s x)-\partial_{t} J_{\beta}(t, s x)\right]+c(t),
\end{gathered}
$$

$\varphi(t, x)$ e $c(t)$ são funções artbitrárias. Assim mostramos que os sistemas físicos com equações de movimento não lagrangianas, de fato, são equivalentes aos sistemas lagrangianos de primeiro ordem. O procedimento proposto para a construção do princípio variacional é ilustrado pelo exemplo de sistemas dinâmicos lineares com equações de movimento lineares não-homogêneas arbitrárias. Construímos uma ação quadrática para estes sistemas.

O material desse capítulo foi publicado no artigo: D.M. Gitman and V.G. Kupriyanov, On the action principle for a system of differential equations, J.Phys.A:Math.Theor.40 (2007) 10071-10081.

No capítulo 3, discutimos o problema da construção do princípio da ação mínima para equação relativistica de Lorentz-Dirac que descreve o movimento de uma partícula carregada no campo eletromagnético com backreaction de radiação (radiation backreaction). Na seção 3.2, é provado que a equação relativistica de Lorentz-Dirac [71], [72], [73]

$$
g_{\mu}=-m \ddot{x}_{\mu}+\frac{e}{c} F_{\mu \nu} \dot{x}^{\nu}+\frac{2 e^{2}}{3 c^{3}}\left(\dddot{x}_{\mu}-\frac{1}{c^{2}} \dot{x}_{\mu} \ddot{x}_{\nu} \ddot{x}^{\nu}\right)=0
$$


bem como seu limite não relativístico

$$
\mathrm{g}=-m \ddot{\mathrm{x}}+e \mathbf{E}+\frac{e}{c}[\dot{\mathrm{x}}, \mathbf{H}]+\frac{2 e^{2}}{3 c^{3}} \dddot{\mathbf{x}}=0,
$$

onde $x^{\mu}=(t, \mathrm{x})$ são coordenadas da partícula e $F_{\mu \nu}=(\mathbf{E}, \mathbf{H})$ é tensor do campo eletromagnético, não admite a existência de um multiplicador de integração e por isso não pode ser obtida pelo princípio da ação mínima. Na seção 3.3, propomos uma equação reduzida de Lorentz-Dirac, isto é, uma equação de segunda ordem que descreve a dinâmica efetiva da carga elétrica com radiação e não tem soluções não-físicas. Na seção 3.3.2, discutimos o problema inverso do cálculo de variações para a equação reduzida de Lorentz-Dirac. Para esta equação, o problema mencionado pode ter várias soluções. Como exemplo foi considerada uma carga pontual não relativística no campo magnético constante e homogêneo $\mathbf{H}=(0,0, H)$ com radiação:

$$
\begin{aligned}
& \ddot{x}=-\alpha \dot{x}-\beta \dot{y}, \\
& \ddot{y}=\beta \dot{x}-\alpha \dot{y}, \\
& \ddot{z}=0,
\end{aligned}
$$

onde usamos o sistema de unidades $m=c=1 \mathrm{e}$

$$
\begin{aligned}
& \alpha=\frac{\sqrt{6} \sqrt{3+\sqrt{9+64 e^{6} H^{2}}}-6}{8 e^{2}} \approx \frac{2}{3} e^{4} H^{2}, \\
& \beta=\frac{e H \sqrt{6}}{\sqrt{3+\sqrt{9+64 e^{6} H^{2}}}} \approx e H .
\end{aligned}
$$

Mostra-se que este sistema admite a existência de um funcional de ação no formalismo de segunda ordem, mas nenhuma das Lagrangianas possíveis transforma-se no limite do desligamento da interação $e \rightarrow 0$ na Lagrangiana usual da partícula livre $L=m \dot{\mathbf{x}} / 2$. Na seção 3.4, uma descrição física satisfatória é obtida para um sistema equivalente de equações diferenciais de primeira ordem.

O material desse capítulo foi publicado no artigo: V.G. Kupriyanov, hamiltonian formulation and action principle for the Lorentz-Dirac system, Int.J.Theor.Phys 45 (2006) 1129.

No capítulo 4, discutimos o problema da constução de tais teorias quânticas que reproduzem equações de movimento não-Lagrangianas para as valores médios no limite clássico. De fato, consideramos a quantização canônica de teorias lagrangianas com vínculos dependentes do tempo que estão relacionadas com teorias não-Lagrangianas. Na seção 4.2, mostramos que a hamiltonização da ação Lagrangiana de primeira ordem que foi construída no capítulo 2 para sistemas não-Lagrangianos conduz a uma teoria hamiltoniana com vínculos de segunda classe dependentes do tempo. Assim, mostramos que os sistemas físicos com equações de movimento não-Lagrangianas, de fato, são equivalentes às teorias lagrangianas de primeira ordem com vínculos dependentes do tempo na formulação hamiltoniana. A quantização canônica de tais 
teorias é um problema não trivial, descrito na seção 4.3. Na seção 4.4, o esquema da quantização proposta é aplicado à construção da teoria quântica de sistemas dinâmicos lineares. Provamos que a evolução quântica dos sistemas considerados é completamente determinada pela evolução clássica correspondente. Depois, consideramos a quantização de exemplos físicos interessantes. Na seção 4.5, o procedimento de quantização canônica de sistemas não-Lagrangianos é ilustrado em detalhes pelo exemplo do oscilador harmônico amortecido. Na seção 4.6, construímos a quantização da carga elétrica com radiação (radiating point-like charge) no campo magnético homogêneo. Foram obtidas as expressões exatas da evolução de valores médios da energia nos exemplos considerados. No caso do segundo exemplo, os valores médios da energia tem a forma:

$$
\langle E\rangle^{t}=\hbar \beta\left(n+\frac{1}{2}\right) e^{-2 \alpha t}, \quad n=0,1, \ldots
$$

Em cada instante fixo o espectro de energia é discreto, contudo ele diminui com o tempo, da mesma forma que.

O material desse capítulo foi publicado nos artigos: D.M. Gitman and V.G. Kupriyanov, Quantization of theories with non-lagrangian equations of motion, Journal of Mathematical Sciences 141 (2007) 1399, a verção curta e D.M. Gitman and V.G. Kupriyanov, Canonical quantization of so-called non-lagrangian systems, Eur.Phys.J.C 50 (2007) 691-700, verção mais elaborada com exemplos físicos.

No capítulo 5, elaboramos um novo método para a construção de ações $\theta$-modificadas na mecânica quântica. Construímos ações $\theta$-modificadas para partículas relativísticas com e sem spin. A idéia chave é extrair as ações $\theta$-modificadas de partículas relativísticas da representação via integral de trajetória dos propagadores da teoria do campo não-comutativa correspondente. Consideramos equações $\theta$-modificadas de Klein-Gordon e de Dirac no campo externo para propagadores causais em tais teorias. Depois, usando o método desenvolvido no artigo [74] para um caso usual comutativo, construímos para estes propagadores a representação via integral de trajetória. Para partícula escalar

$$
\begin{aligned}
& D^{c}=i \int_{0}^{\infty} d \lambda_{0} \int_{x_{i n}-\theta p / 2 \hbar}^{x_{\text {out }}-\theta p / 2 \hbar} D q \int_{\lambda_{0}} D \lambda \int D p D \pi \exp \left\{\frac{i}{\hbar} S_{\text {scal-part }}^{\theta}+S_{\mathrm{GF}}\right\}, \\
& S_{\mathrm{scal}-\text { part }}^{\theta}=\int_{0}^{1}\left\{\lambda\left[\left(p_{\mu}+g A_{\mu}(q)\right)^{2}-m^{2}\right]+p_{\mu} \dot{q}^{\mu}+\frac{1}{2 \hbar} \dot{p}_{\mu} \theta^{\mu \nu} p_{\nu}\right\} d \tau, \\
& S_{\mathrm{GF}}=\int_{0}^{1} \pi \dot{\lambda} d \tau .
\end{aligned}
$$


Para partícula spinorial

$$
\begin{aligned}
& \tilde{G}^{c}=\exp \left(i \Gamma^{n} \frac{\partial_{l}}{\partial \varepsilon^{n}}\right) \int_{0}^{\infty} d \lambda_{0} \int d \chi_{0} \int_{\lambda_{0}} D \lambda \int_{\chi_{0}} D \chi \int_{-\infty}^{\infty} D p \int_{x_{\text {in }}-\theta p / 2 \hbar}^{x_{\text {out }}-\theta p / 2 \hbar} D q \int D \pi \int D \nu \\
& \times\left.\int_{\psi(0)+\psi(1)=\varepsilon} \mathcal{D} \psi \exp \left\{i\left[S_{\text {spin-part }}^{\theta}+S_{\mathrm{GF}}\right]+\psi_{n}(1) \psi^{n}(0)\right\}\right|_{\varepsilon=0},
\end{aligned}
$$

onde

$$
\begin{aligned}
& S_{\text {spin-part }}^{\theta}=\int_{0}^{1}\left[\lambda\left(\left(p_{\mu}+g A_{\mu}\right)^{2}-m^{2}+2 i g F_{\mu \nu}^{*} \psi^{\mu} \psi^{\nu}\right)\right. \\
& \left.+2 i\left(p_{\mu}+g A_{\mu}(q)\right) \psi^{\mu} \chi-2 i m \psi^{D} \chi-i \psi_{n} \dot{\psi}^{n}+p_{\mu} \dot{q}^{\mu}+\frac{1}{2 \hbar} \dot{p}_{\mu} \theta^{\mu \nu} p_{\nu}\right] d \tau \\
& S_{\mathrm{GF}}=\int_{0}^{1}(\pi \dot{\lambda}+\nu \dot{\chi}) d \tau .
\end{aligned}
$$

As ações $S_{\text {scal }}^{\theta}$ e $S_{\text {spin-part }}^{\theta}$ em (1.13) e (1.14) distinguem-se dos casos comutativos correspondentes (veja [74]) pelo termo $\dot{p}_{\mu} \theta^{\mu \nu} p_{\nu} / 2 \hbar$. Tratamos as ações efetivas nesta representação como ações $\theta$-modificadas de partículas relativísticas. Para confirmar a interpretação, construímos a quantização canônica das ações obtidas. Assim, obtivemos as equações $\theta$-modificadas de KleinGordon e de Dirac na teoria de campo não-comutativa. A ação $\theta$-modificada para a partícula relativistica com spin (1.14) é uma generalização da ação pseudoclássica de Berezin-Marinov [68] para caso não-comutativo. Note que os efeitos da não comutatividade são essenciais somente no caso da presença do campo externo.

O material desse capítulo foi publicado no artigo: D.M. Gitman and V.G. Kupriyanov, Path integral representations in noncommutative quantum mechanics and noncommutative version of Berezin-Marinov action, Eur.Phys.J.C 54 (2008) 325.

No capítulo 6, desenvolvemos uma abordagem para a quantização por deformação no espaço plano real com uma estrutura de Poisson $\omega^{i j}(x)$ arbitrária baseada no ordenamento simétrico dos operadores $\hat{x}^{i}$, que representam coordenadas cartesianas deformadas no $\mathbb{R}^{N}$. Impusemos relações de comutação gerais $\left[\hat{x}^{i}, \hat{x}^{j}\right]=2 \alpha \omega^{i j}(\hat{x})$ (onde $\alpha$ é o parâmetro da deformação) para os $\hat{x}^{i}$ e então construímos a representação polidiferencial para $\hat{x}^{i}=x^{i}+\alpha \omega^{i j}(x) \partial_{j}+\ldots$. Usando esta representação, conseguimos formular um procedimento iterativo simples e efetivo que permitiu calcular o produto estrela em ordens altas (em terceira e quarta ordens e pode ser estendida até a quinta ordem), algo que foi feito pela primeira vez. Exceto por uma análise da cohomologia, que não consideramos no artigo, o método proposto dá uma descrição explicita, na linguagem matemática usual da física, do produto estrela.

Gostaríamos de notar que, em nosso procedimento, o cálculo do produto estrela em ordens altas é feito com facilidade, com excessão da solução da condição de consistência para a construção das correções "não Poisson" $\omega_{2 n}^{i j}(x)$ para o bi-vetor de Poisson $\omega_{0}^{i j}(x)$, para a qual não 
pudemos apresentar um algoritmo geral. As equações para $\omega_{2 n}^{i j}(x)$ que aparecem na nossa abordagem são praticamente idênticas às que surgem da abordagem por cohomologias de Hochschild [75]. O estudo das relações entre a nossa abordagem e a abordagem baseada em cohomologias de Hochschild é um problema interessante e nós planejamos fazê-lo no futuro.

Contudo, em alguns casos (e.g., caso bi-dimensional ou algumas estruturas de Poisson polinomiais, como a álgebra de Lie), a condição de consistência resolve-se automaticamente, e os cálculos do produto estrela podem ser feitos em ordem arbitrária. Podemos, assim, inclusive tentar deduzir uma expressão exata do produto estrela nestes casos.

O material deste capítulo foi publicado no artigo: V.G. Kupriyanov, D.V. Vassilevich, Star products made (somewhat) easier, Eur.Phys.J.C. 58 (2008) 627-637. 


\section{Capítulo 2}

\section{Princípio de ação mínima para equações diferenciais}

\subsection{Introdução}

O problema da construção do princípio de ação mínima para equações de movimento determinadas é conhecido na literatura como o problema inverso do cálculo de variações da mecânica Newtoniana. Este problema em sua definição clássica [4] consiste em resolver a equação variacional

$$
\frac{\delta S[q]}{\delta q^{i}(t)}=g_{i}
$$

onde $g_{i}\left(t, q^{i}, \dot{q}^{i}, \ldots\right)=0$ é um dado sistema de equações diferenciais em relação às funções $q^{i}(t)$, e $S[q]$ é um funcional local a ser determinado. A condição da localidade requer a existência de uma função $L(t, q, \dot{q} \ldots)$ (Lagrangiana), tal que o funcional $S[q]$ (ação) seria escrito como um integral

$$
S[q]=\int d t L
$$

Em outras palavras, a essência do problema inverso do cálculo das variações consiste em encontrar um princípio variacional para um determinado sistema de equações diferenciais. Este problema tem sido considerado por mais de um século. Ainda no ano 1887 Helmholtz [4] propôs um critério de resolubilidade da equação (2.1):

$$
\frac{\delta g_{i}(t)}{\delta q^{j}(s)}=\frac{\delta g_{j}(s)}{\delta q^{i}(t)}
$$

Caso essa condição seja obedecida, o sistema $g_{i}\left(t, q^{i}, \dot{q}^{i}, \ldots\right)=0$ se chama um sistema Lagrangiano, caso contrário o sistema é um sistema não-Lagrangiano. Em 1894 Darboux [5] resolveu o problema inverso do cálculo de variações no caso unidimensional. O caso bidimensional foi investigado por Douglas [6] em 1941. Em particular, ele apresentou exemplos de equações 
diferenciais de segunda ordem que não podem ser obtidas do princípio de ação mínima (nenhuma Lagrangiana existe para tais equações). Depois disso vários autores [7]-[19] investigaram o problema da construção de princípio variacional para sistemas multidimencionais.

No presente capítulo, consideramos a questão da construção de um princípio de ação minima para um determinado sistema de equações diferenciais, utilizando o método do multiplicador de integração [6]-[11]. O multiplicador de integração é uma matriz multiplicadora que pode dotar um sistema de equações diferenciais com a estrutura das equações de Euler-Lagrange. Apresentamos na seção 2 um método simples da construção de condições necessárias e suficientes para a existência do multiplicador de integração para equações de segunda ordem. Caso o multiplicador de integração exista e é conhecido, nós apresentamos uma forma explícita da Lagrangiana. Na seção 3 o método proposto da solução do problema inverso do cálculo de variações é ilustrado por exemplos diferentes. Será construida a ação Lagrangiana no formalismo de segunda ordem de sistemas dissipativos multidimensionais. Consideramos também o exemplo do sistema dinâmico linear com equações de movimento que não admitem a existência do multiplicador de integração.

Note que um sistema de $n$ equações não-Lagrangianas de segunda ordem sempre pode ser apresentado na forma de um sistema equivalente de $2 n$ equações diferenciais de primeira ordem. Partindo do critério de Helmholtz (2.3) encontramos condições necessárias e suficientes para a existência de um multiplicador de integração para essas equações. No formalismo de primeira ordem o multiplicador de integração sempre existe e pode ser construído por meio da solução do problema de Cauchy para as equações consideradas. Isto é apresentado na seção 3, e é parcialmente baseado nos resultados do trabalho [12]. Depois construímos a ação explicitamente. Desta forma, mostramos que aos sistemas tradicionalmente denominados de não-Lagrangianos são, na realidade, equivalentes aos sistemas Lagrangianos de primeira ordem. Como exemplo, construimos um funcional da ação de primeira ordem para qualquer sistema dinâmico linear.

\subsubsection{Princípio de ação mínima para o sistema das equações da se- gunda ordem}

\subsubsection{Considerações gerais}

Vamos supor que um sistema físico com $n$ graus de liberdade é descrito por um sistema de $n$ equações diferenciais de segunda ordem

$$
\ddot{q}^{i}-f^{i}(t, q, \dot{q})=0, \quad i=1 \ldots n,
$$

onde $f^{i}(t, q, \dot{q})$ são algumas funções. Vamos construir um princípio de ação mínima para este sistema. Caso as equações (2.4) não possam ser diretamente identificadas com as equações de Euler-Lagrange, podemos procurar um multiplicador de integração, ou seja, uma matriz não singular $h_{i j}(t, q, \dot{q})$ tal que após a multiplicação por (2.4)

$$
h_{i j}\left[\ddot{q}^{j}-f^{j}(t, q, \dot{q})\right]=0
$$


transformaria essas equações na forma de equações de Euler-Lagrange para uma função de Lagrange $L(t, q, \dot{q})$,

$$
\frac{\partial L}{\partial q^{i}}-\frac{\partial^{2} L}{\partial t \partial \dot{q}^{i}}-\frac{\partial^{2} L}{\partial \dot{q}^{i} \partial q^{j}} \dot{q}^{j}-\frac{\partial^{2} L}{\partial \dot{q}^{i} \partial \dot{q}^{j}} \ddot{q}^{j}=0
$$

Para identificar as equações (2.5) e (2.6) precisamos que as condições

$$
\begin{aligned}
& \frac{\partial^{2} L}{\partial \dot{q}^{i} \partial \dot{q}^{j}}=h_{i j}, \\
& \frac{\partial L}{\partial q^{i}}-\frac{\partial^{2} L}{\partial t \partial \dot{q}^{i}}-\frac{\partial^{2} L}{\partial \dot{q}^{i} \partial q^{j}} \dot{q}^{j}=h_{i j} f^{j} .
\end{aligned}
$$

sejam obedecidas. Quando o multiplicador de integração é conhecido, as equações (2.7)-(2.8) podem ser interpretadas como um sistema de equações para a função de Lagrange $L$. Vamos resolver o sistema (2.7)-(2.8). As suas condições de consistência nos fornecerá todas as condições necessárias e suficientes para a existência do multiplicador de integração. Partindo do pressuposto de que $L(t, q, \dot{q})$ é uma função suave dos argumentos indicados, as condições de consistência da equação (2.7) implicam que

$$
h_{i j}=h_{j i}, \quad \frac{\partial h_{i j}}{\partial \dot{q}^{k}}=\frac{\partial h_{k j}}{\partial \dot{q}^{i}} .
$$

Caso $h_{i j}$ obedece (2.9), a equação (2.7) pode ser resolvida.

Vamos lembrar que a solução geral da equação $\partial f / \partial q^{i}=g_{i}$, desde que o vetor $g_{i}$ é um gradiente, é

$$
f(q)=\int_{0}^{1} d s q^{i} g_{i}(s q)+c
$$

onde $c$ é uma constante. Tendo em vista o fato acima, obtém-se para $L$ (aqui não consideramos os problemas globais que podem surgir quando o espaço das configurações tem uma topologia não trivial) a seguinte representação:

$$
L=K(t, q, \dot{q})+l_{i}(t, q) \dot{q}^{i}+l_{0}(t, q)
$$

onde

$$
K(t, q, \dot{q})=\int_{0}^{1} d a \dot{q}^{j}\left[\int_{0}^{1} d b \dot{q}_{1}^{i} h_{i j}\left(t, q, b \dot{q}_{1}\right)\right]_{\dot{q}_{1}=a \dot{q}}
$$

e $l_{0}(t, q), l_{i}(t, q)$ são algumas funções. Para encontrar essas funções, usamos equação (2.8). Substituindo (2.10) em (2.8), obtemos

$$
\frac{\partial K}{\partial q^{i}}-\frac{\partial^{2} K}{\partial \dot{q}^{i} \partial t}-\frac{\partial^{2} K}{\partial \dot{q}^{i} \partial q^{j}} \dot{q}^{j}+\left(\frac{\partial l_{j}}{\partial q^{i}}-\frac{\partial l_{i}}{\partial q^{j}}\right) \dot{q}^{j}-\frac{\partial l_{i}}{\partial t}+\frac{\partial l_{0}}{\partial q^{i}}=h_{i j} f^{j}
$$


Diferenciando esta equação sobre $\dot{q}^{k}$, obtemos:

$$
\frac{\partial l_{k}}{\partial q^{i}}-\frac{\partial l_{i}}{\partial q^{k}}=L_{i k}
$$

onde

$$
L_{i k}=\frac{\partial^{2} K}{\partial \dot{q}^{i} \partial q^{j}}-\frac{\partial^{2} K}{\partial \dot{q}^{j} \partial q^{i}}+\frac{\partial h_{i k}}{\partial t}+\dot{q}^{j} \frac{\partial h_{i k}}{\partial q^{j}}+\frac{\partial}{\partial \dot{q}^{k}}\left(h_{i j} f^{j}\right) .
$$

Esta equação é uma equação diferencial para $l_{i}$. As condições da consistência da equação (2.13) implicam que: em primeiro lugar, a parte simetrica da matriz $L_{i k}$ deve ser igual a zero, que pode ser escrita como

$$
\hat{D} h_{i k}+\frac{1}{2}\left(h_{i j} \frac{\partial f^{j}}{\partial \dot{q}^{k}}+h_{k j} \frac{\partial f^{j}}{\partial \dot{q}^{i}}\right)=0
$$

onde

$$
\hat{D}=\frac{\partial}{\partial t}+\dot{q}^{j} \frac{\partial}{\partial q^{j}}+f^{j} \frac{\partial}{\partial \dot{q}^{j}} .
$$

Usando (2.15), podemos reescrever (2.14) como

$$
L_{i k}=\frac{\partial^{2} K}{\partial \dot{q}^{i} \partial q^{k}}-\frac{\partial^{2} K}{\partial \dot{q}^{k} \partial q^{i}}+A_{i k}, \quad A_{i k}=\frac{1}{2}\left(h_{i j} \frac{\partial f^{j}}{\partial \dot{q}^{k}}-h_{k j} \frac{\partial f^{j}}{\partial \dot{q}^{i}}\right)
$$

Em segundo lugar, $L_{i k}$ não pode depender da velocidade, ou seja, $\partial L_{i k} / \partial \dot{q}^{l}=0$, que implica

$$
\frac{\partial h_{k l}}{\partial q^{i}}-\frac{\partial h_{i l}}{\partial q^{k}}=\frac{\partial}{\partial \dot{q}^{l}} A_{i k}
$$

E finalmente, a identidade de Jacobi para $L_{i k}$ :

$$
\frac{\partial L_{i k}}{\partial q^{l}}+\frac{\partial L_{k l}}{\partial q^{i}}+\frac{\partial L_{l i}}{\partial q^{k}}=0 \Rightarrow \frac{\partial A_{i k}}{\partial q^{l}}+\frac{\partial A_{k l}}{\partial q^{i}}+\frac{\partial A_{l i}}{\partial q^{k}}=0 .
$$

Desde que $h_{i j}$ obedeça as equações $(2.15),(2.17)$ e $(2.18), l_{i}$ pode ser encontrada da equação (2.13). Lembramos que a solução geral $l_{i}$ da equação (2.13) é dada por

$$
l_{i}(t, q)=\int_{0}^{1} d a q^{k} L_{k i}(t, a q)+\frac{\partial \varphi(t, q)}{\partial q^{i}}
$$

onde $\varphi(t, q)$ é uma função arbitrária.

Agora, podemos encontrar $l_{0}$ da equação (2.12); para isso vamos reescreve-lo na seguinte forma:

$$
\frac{\partial l_{0}}{\partial q^{i}}=m_{i}
$$


onde

$$
m_{i}=h_{i j} f^{j}-\frac{\partial K}{\partial q^{i}}+\frac{\partial^{2} K}{\partial t \partial \dot{q}^{i}}+\dot{q}^{j} \frac{\partial^{2} K}{\partial q^{j} \partial \dot{q}^{i}}-\dot{q}^{j} L_{i j}+\frac{\partial l_{i}}{\partial t} .
$$

A condição da consistência da (2.20) implica que, $m_{i}$ não depende da velocidade, ou seja, $\partial m_{i} / \partial \dot{q}^{k}=0$. Esta condição é fornecida pela equação (2.15). Além disso, o vetor $m_{i}$ tem que ser um vetor gradiente:

$$
\frac{\partial m_{i}}{\partial q^{k}}-\frac{\partial m_{k}}{\partial q^{i}}=\frac{\partial A_{i k}}{\partial t}+\dot{q}^{j} \frac{\partial A_{i k}}{\partial q^{j}}+\frac{\partial}{\partial q^{k}}\left(h_{i j} f^{j}\right)-\frac{\partial}{\partial q^{i}}\left(h_{k j} f^{j}\right)=0 .
$$

Tendo em conta (2.9), (2.15) e (2.17), obtemos da (2.22) a seguinte condição algébrica:

$$
h_{i j} B_{k}^{j}-h_{k j} B_{i}^{j}=0,
$$

onde

$$
B_{j}^{i}=\frac{1}{2} \frac{\partial f^{i}}{\partial \dot{q}^{m}} \frac{\partial f^{m}}{\partial \dot{q}^{j}}-\hat{D} \frac{\partial f^{i}}{\partial \dot{q}^{j}}+2 \frac{\partial f^{i}}{\partial q^{j}} .
$$

Caso $h_{i j}$ obedeça (2.23), da equação (2.20) obteremos

$$
l_{0}(t, q)=\int_{0}^{1} d a q^{k} m_{k}(t, a q)+\frac{\partial \varphi(t, q)}{\partial t}+c(t),
$$

onde $c(t)$ é uma função arbitrária do tempo.

Assim, provamos a seguinte afirmação: se para um determinado sistema de equações diferenciais ordinárias de segunda-ordem (2.4) existe uma matriz não-singular $h_{i j}(t, q, \dot{q})$ que obedece as equações (2.9), (2.15), (2.17), (2.18) e (2.23), neste caso esse sistema pode ser obtido a partir do princípio variacional com Lagrangiano (2.10), onde as funções $K(t, q, \dot{q}), l_{i}(t, q) e$ $l_{0}(t, q)$ são definidas em (2.11), (2.19) e (2.24) respectivamente, e as funções $\varphi(t, q)$ e $c(t)$ são funções arbitrárias.

A arbitrariedade relacionada com as funções $\varphi(t, q)$ e $c(t)$ entre na Lagrangiana (2.10) através da derivada total de uma função $F$,

$$
F=\varphi(t, q)+\int c(t) d t .
$$

Note que o multiplicador de integração $h_{i j}$, e como conseqüência a função de Lagrange $L$ existem não para qualquer um sistema de equações diferenciais (2.4). Na seção 2.2 será considerado um exemplo do sistema dinâmico, que não admite a existência de um multiplicador de integração. Contudo, caso o multiplicador de integração existe, ele não é único [15]-[21]. Por exemplo, se a matriz $h_{i j}$ é um multiplicador de integração para um sistema de equações (2.4), observamos que a matriz $\hat{h}_{i j}=c h_{i j}$, com $c \neq 0$ é uma constante, também é um multiplicador de integração. Portanto, Lagrangiana (2.10) que fornece após a variação as equações (2.4) 
não é úniqa; porque para este sistema existem tantas Lagrangians não-equivalentes quanto multiplicadores de integração. Lagrangianas correspondentes aos diferentes multiplicadores de integração são conhecidos como Lagrangianas $s$-equivalentes.

No caso uni dimensional $\ddot{q}-f(t, q, \dot{q})=0$, o multiplicador de integração é uma função $h(t, q, \dot{q})$ que não pode ser igual a zero e obedece a equação

$$
\frac{\partial h}{\partial t}+\dot{q} \frac{\partial h}{\partial q}+\frac{\partial}{\partial \dot{q}}(f h)=0 .
$$

Este é uma PDE de primeira ordem que, obviamente, tem uma solução para qualquer $f$ e a condição inicial $h(t=0, q, \dot{q})=h_{0}(q, \dot{q})$. Como podemos ver, uma resposta à questão se existe uma solução do problema inverso do cálculo das variações ou não depende do número de graus de liberdade $n$. Para $n=1$ a resposta sempre será positiva, e existem tantas Lagrangianas não-equivalente quanto funções $h_{0}(q, \dot{q})$ das duas variáveis. Para $n \geq 2$ a resposta pode ser negativa.

\subsubsection{Exemplos}

Nesta seção, ilustramos o procedimento acima nos exemplos de sistemas dinâmicos. Primeiramente consideramos os sistemas dissipativos. Consideramos um sistema ideal com Lagrangiana

$$
L_{0}=\frac{\dot{q}^{2}}{2}+V(q), \quad q=\left\{q^{i}\right\}, \quad i=1 \ldots n .
$$

Vamos supor que, além da força potencial conservativa $F^{i}=\frac{\partial V}{\partial q^{i}}$ existe uma força do atrito

$$
F_{\text {fric }}^{i}=\alpha \dot{q}^{i},
$$

com $\alpha$ é um coeficiente de atrito fenomenológico que, em geral, pode depender do tempo. As equações de movimento para um sistema deste tipo têm a forma

$$
\ddot{q}^{i}=\frac{\partial V}{\partial q^{i}}+\alpha \dot{q}^{i}
$$

Estas equações são equações não-Lagrangianas, mas para este sistema sempre é possível encontrar um multiplicador de integração. No caso mais simples, quando ele não depende das coordenadas e velocidades, tem a forma

$$
h_{i j}=e^{-2 \int \alpha d t} h_{i j}^{0},
$$

onde $h_{i j}^{0}$ é uma matriz arbitrária simétrica e não-singular, constante que comute com a matriz $V_{i j}=\partial^{2} V / \partial q^{i} \partial q^{j}$. Usando a declaração da seção anterior, obtemos a seguinte Lagrangiana:

$$
L=\frac{1}{2} \dot{q}^{i} h_{i j} \dot{q}^{j}+\int_{0}^{1} q^{i} h_{i j} \frac{\partial V(s \vec{q})}{\partial q^{j}} d s .
$$


Se escolher $h_{i j}^{0}=\delta_{i j}$, Lagrangiana (2.30) pode ser reescrita como

$$
L=e^{-2 \int \alpha d t} L_{0}
$$

Note que quando o coeficiente de atrito chega a zero, Lagrangiana (2.31) se transforma na Lagrangiana inicial (2.26).

Agora, consideramos o exemplo de um sistema dinâmico para o qual o multiplicador de integração e, conseqüentemente, a possibilidade da descrição Lagrangiana não existe. Duglas [6] mostrou que o sistema de equações de segunda ordem

$$
\begin{aligned}
& \ddot{x}+\dot{y}=0, \\
& \ddot{y}+y=0
\end{aligned}
$$

não admite um multiplicador de integração. Para provar isso, vamos supor o contrário, ou seja, que haja uma matriz não-singular $h_{i j}$ que obedeça as equações $(2.9),(2.15),(2.17),(2.18) \mathrm{e}$ (2.23). Assim, da equação algébrica (2.23) segue-se que $h_{i j}$ tem que ser diagonal $\left(h_{12}=h_{21}=0\right)$, já que neste caso

$$
B_{j}^{i}=\left(\begin{array}{ll}
0 & 0 \\
0 & 2
\end{array}\right)
$$

Depois, da condição (2.15) vamos obter imediatamente que $h_{11}=0$, e chegar a uma contradição, $\operatorname{det} h_{i j}=0$.

Assim, podemos ver que um funcional de ação no formalismo de segunda ordem nem sempre existe. No entanto, como mostraremos na seção seguinte, sempre é possível construir um princípio de ação mínima para um sistema equivalente de equações de primeira ordem.

\subsection{Princípio de ação mínima no formalismo de primeira ordem}

Vamos supor que um sistema com $n$ graus de liberdade descrita por um sistema de $n$ equações diferenciais não-Lagrangianas de segunda ordem. Para construir um princípio de ação minima, vamos substituir essas equações (que sempre é possível fazer através da introdução $n$ variáveis adicionais, como por exemplo $p_{i}=\dot{q}^{i}$ ) para um sistema equivalente de $2 n$ equações diferenciais da primeira ordem. Suponhamos que este sistema tem a forma

$$
\begin{aligned}
& \dot{x}^{\alpha}=f^{\alpha}(t, x), \\
& x^{\alpha}=\left(q^{i}, p_{i}\right), \quad \alpha=1, . ., 2 n,
\end{aligned}
$$

onde $f^{\alpha}(t, x)$ são algumas funções dos argumentos indicados. Vamos construir um princípio de ação para este sistema. Se (2.32) não podem ser diretamente identificados com as equações de 
Euler-Lagrange, é possível encontrar um multiplicador de integração, ou seja, uma matriz nãosingular $\Omega^{1}$ que transforma o sistema inicial de equações diferenciais (2.32) em uma derivada funcional:

$$
g_{\alpha}[t]=\Omega_{\alpha \beta}\left(\dot{x}^{\beta}-f^{\beta}(t, x)\right)=\frac{\delta S}{\delta x^{\alpha}}=0 .
$$

Como $g_{\alpha}[t]$ é um derivado variacional ele tem que obedecer o criterio de Helmholtz [4]

$$
\frac{\delta g_{\alpha}[t]}{\delta x^{\beta}(s)}=\frac{\delta g_{\beta}[s]}{\delta x^{\alpha}(t)} .
$$

Vamos usar esta condição para encontrar um multiplicador de integração $\Omega$. No caso geral pode-se assumir que $\Omega$ depende do tempo $t$, coordenadas $x^{\alpha}$ e derivadas de $x^{\alpha}$ em relação ao tempo até a ordem $m$ ( $m$ é um número natural), ou seja, $g_{\alpha}[t]=g_{\alpha}\left(t, x, \ldots, x^{(m)}\right)$. Tendo em mente essa forma de $g_{\alpha}$ pode-se reescrever (2.34) como

$$
\sum_{i=0}^{m} \frac{\partial g_{\alpha}[t]}{\partial x^{\beta(i)}} \delta^{(i)}(t-s)=\sum_{j=0}^{m} \frac{\partial g_{\beta}[s]}{\partial x^{\alpha(j)}} \delta^{(j)}(s-t)
$$

já que

$$
\frac{\delta}{\delta x^{\beta}(s)} x^{\alpha(k)}(t)=\left(\frac{d}{d t}\right)^{k} \frac{\delta x^{\alpha}(t)}{\delta x^{\beta}(s)}=\delta_{\beta}^{\alpha} \delta^{(k)}(t-s), \quad k=0,1, \ldots
$$

Diferenciando a identidade $f(t) \delta(t-s)=f(s) \delta(s-t)$ por $t$ encontra-se

$$
f(s) \delta^{(k)}(s-t)=(-1)^{k} \sum_{l=0}^{k} C_{l}^{k} f^{(l)}(t) \delta^{(k-l)}(t-s), \quad C_{l}^{k}=\frac{k !}{(k-l) !} .
$$

Usando (2.36) reescrevemos (2.34) como

$$
\sum_{i=0}^{m} \frac{\partial g_{\alpha}[t]}{\partial x^{\beta(i)}} \delta^{(i)}(t-s)=\sum_{j=0}^{m}(-1)^{j} \sum_{l=0}^{j} C_{l}^{j}\left[\left(\frac{d}{d t}\right)^{l} \frac{\partial g_{\beta}[t]}{\partial x^{\alpha(j)}}\right] \delta^{(j-l)}(t-s) .
$$

Comparando em (2.37) as coeficientes na frente de $\delta^{(k)}(t-s)$ obtemos as equações para $g_{\alpha}\left(t, x, \ldots, x^{(m)}\right)$. Quando $k=0$ temos

$$
\frac{\partial g_{\alpha}}{\partial x^{\beta}}-\sum_{j=0}^{m}(-1)^{j}\left(\frac{d}{d t}\right)^{l} \frac{\partial g_{\beta}}{\partial x^{\alpha(j)}}=0 .
$$

Como $g_{\alpha}[t]$ depende somente das derivadas até a ordem $m$, o coeficiente da derivada mais alta $x^{\alpha(2 m)}$ na equação (2.38) tem que zerar. Este coeficiente é $(-1)^{m} \partial^{2} g_{\beta} / \partial x^{\alpha(m)} \partial x^{\gamma(m)}$, que significa que $g_{\alpha}[t]$ deve ser linear pelas derivadas de ordem $m$, ou seja,

$$
g_{\alpha}[t]=a_{\alpha \beta}\left(t, x, \ldots, x^{(m-1)}\right) x^{\beta(m)}+b_{\alpha}\left(t, x, \ldots, x^{(m-1)}\right),
$$

\footnotetext{
${ }^{1}$ Notamos por $\Omega$ um multiplicador de integração para as equações da primeira ordem.
} 
onde $a_{\alpha \beta}$ e $b_{\alpha}$ são algumas funções. Já que $\Omega$ é uma matriz não-singular, (2.33) deve ser um sistema de equações de primeira ordem, ou seja, temos $m=1$ e $\Omega=\Omega(t, x)$.

Agora comparando o coeficiente de $\delta^{(1)}(t-s)$ em (2.37), encontramos:

$$
\Omega_{\alpha \beta}=-\Omega_{\beta \alpha} .
$$

Depois da equação (2.38) temos

$$
\partial_{\beta}\left(\Omega_{\alpha \gamma} f^{\gamma}\right)-\partial_{\alpha}\left(\Omega_{\beta \gamma} f^{\gamma}\right)+\partial_{t} \Omega_{\alpha \beta}+\dot{x}^{\gamma}\left(\partial_{\beta} \Omega_{\alpha \gamma}-\partial_{\alpha} \Omega_{\beta \gamma}+\partial_{\gamma} \Omega_{\beta \alpha}\right)=0 .
$$

Como $\Omega$ não depende de $\dot{x}$ encontramos as equações seguintes para $\Omega$ :

$$
\partial_{\alpha} \Omega_{\beta \gamma}+\partial_{\beta} \Omega_{\gamma \alpha}+\partial_{\gamma} \Omega_{\alpha \beta}=0
$$

$\mathrm{e}$

$$
\partial_{t} \Omega_{\alpha \beta}+£_{f} \Omega_{\alpha \beta}=0,
$$

com $£_{f} \Omega_{\alpha \beta}$ sendo a derivada de Lie da $\Omega_{\alpha \beta}$ ao longo do campo vetorial $f^{\gamma}$ e $\partial_{\alpha}=\partial / \partial x^{\alpha}, \partial_{t}=$ $\partial / \partial t$. Assim, vemos que para um sistema de equações de primeira ordem o multiplicador de integração é uma matriz não-singular que depende apenas do tempo $t$ e das coordenadas $x^{\alpha}$, e obedece as condições (2.39), (2.40a) e (2.41).

Vamos analisar as equações (2.39)-(2.41) para a matriz $\Omega_{\alpha \beta}$. Sabe se que a solução geral $\Omega_{\alpha \beta}$ da equação (2.41) pode ser construida pela solução do problema de Cauchy de equações (2.32). Suponha que tal solução é conhecida:

$$
x^{\alpha}=\varphi^{\alpha}\left(t, x_{(0)}\right), x_{(0)}^{\alpha}=\varphi^{\alpha}\left(0, x_{(0)}\right)
$$

é uma solução de equações (2.32) para qualquer $x_{(0)}=\left(x_{(0)}^{\alpha}\right)$, e $\chi^{\alpha}(t, x)$ é a função inversa em relação à $\varphi^{\alpha}\left(t, x_{(0)}\right)$, ou seja,

$$
x^{\alpha}=\varphi^{\alpha}\left(t, x_{(0)}\right) \Longrightarrow x_{(0)}^{\alpha}=\chi^{\alpha}(t, x), x^{\alpha} \equiv \varphi^{\alpha}\left(t, \chi^{\alpha}\right),\left.\partial_{\alpha} \chi^{\gamma}\right|_{t=0}=\delta_{\gamma}^{\alpha} .
$$

Assim

$$
\Omega_{\alpha \beta}(t, x)=\partial_{\alpha} \chi^{\gamma} \Omega_{\gamma \delta}^{(0)}(\chi) \partial_{\beta} \chi^{\delta}
$$

com a matriz $\Omega_{\alpha \beta}^{(0)}$ sendo a condiçãao inicial para $\Omega_{\alpha \beta}$,

$$
\left.\Omega_{\alpha \beta}(t, x)\right|_{t=0}=\Omega_{\alpha \beta}^{(0)}(x)
$$

Podemos ver que ao escolher a matriz $\Omega_{\alpha \beta}^{(0)}(x)$ sendo não-singular e sujeito a identidade de Jacobi, garantimos o cumprimento das mesmas condições para a matriz completa $\Omega_{\alpha \beta}(t, x)$, já que os componentes dela são determinados por uma mudança de variáveis (2.44). 
Assim, vemos que para qualquer um sistema de equações da primeira ordem (2.32), o multiplicador de integração sempre existe, ou seja, sempre existe uma Lagrangiana $L(t, x, \dot{x})$ que tem o sistema de equações

$$
\Omega_{\alpha \beta}\left(\dot{x}^{\beta}-f^{\beta}(t, x)\right)=0
$$

como suas equações de Euler-Lagrange. É fácil ver que esta Lagrangiana deve ser linear pelos derivados da primeira ordem $\dot{x}^{\alpha}$ já que as equações (2.45) não contêm derivados de segunda ordem, ou seja, o termo correspondente $\partial^{2} L / \partial \dot{x}^{\alpha} \partial \dot{x}^{\beta}$ zera. A forma geral desta Lagrangiana é

$$
L=J_{\alpha} \dot{x}^{\alpha}-H,
$$

com $J_{\alpha}=J_{\alpha}(t, x)$ e $H=H(t, x)$ são algumas funções dos argumentos indicados. As equações de Euler-Lagrange correspondentes a (2.46) são

$$
\frac{\delta S}{\delta x}=\frac{\partial L}{\partial x}-\frac{d}{d t} \frac{\partial L}{\partial \dot{x}}=0 \Longrightarrow-\partial_{\alpha} H-\partial_{t} J_{\alpha}+\left(\partial_{\alpha} J_{\beta}-\partial_{\beta} J_{\alpha}\right) \dot{x}^{\beta}=0 .
$$

Comparando as equações (2.45) e (2.47) encontramos

$$
\Omega_{\alpha \beta}=\partial_{\alpha} J_{\beta}-\partial_{\beta} J_{\alpha},
$$

e

$$
\Omega_{\alpha \beta} f^{\beta}-\partial_{t} J_{\alpha}=\partial_{\alpha} H .
$$

As funções $J_{\alpha}$ e $H$ podem ser encontrados das condições (2.48) e (2.49) se a matriz $\Omega_{\alpha \beta}$ é conhecida. Pode-se ver que as condições de coerência para estas equações nos fornecerá exatamente as equações (2.39)-(2.41) para o multiplicador de integração $\Omega_{\alpha \beta}$. A solução geral $J_{\alpha}(t, x)$ da equação (2.48), desde que $\Omega_{\alpha \beta}$ é uma determinada matriz anti-simétrica que obedece a identidade de Jacobi, é dada por

$$
J_{\alpha}(t, x)=\int_{0}^{1} x^{\beta} \Omega_{\beta \alpha}(t, s x) s d s+\partial_{\alpha} \varphi(t, x),
$$

com $\varphi(x)$ é uma função arbitrária. Substituindo (2.44) em (2.50), obtemos

$$
J_{\alpha}(t, y)=\left.\int_{0}^{1} y^{\beta}\left[\partial_{\alpha} \chi^{\gamma} \Omega_{\gamma \delta}^{(0)}(\chi) \partial_{\beta} \chi^{\delta}\right]\right|_{x=s y} s d s+\partial_{\alpha} \varphi(t, y)
$$

A equação (2.51) descreve todas as ambiguidades (uma arbitrária matriz simpléctica $\Omega_{\gamma \delta}^{(0)}$ e uma função arbitrária $\varphi(t, x))$ na construção do termo $J_{\alpha}(t, x)$ da função de Lagrange (2.46).

Para encontrar o termo $H$ na função de Lagrange (2.46), precisamos resolver a equação (2.49) em relação a $H$. Obtemos para $H$ a seguinte representação:

$$
H(t, x)=\int_{0}^{1} d s x^{\beta}\left[\Omega_{\beta \alpha}(t, s x) f^{\alpha}(t, s x)-\partial_{t} J_{\beta}(t, s x)\right]+c(t),
$$


com $c(t)$ sendo uma função arbitrária do tempo, e $\Omega_{\beta \alpha}$ e $J_{\beta}$ são dadas por (2.44) e (2.51) respectivamente. Todas as arbitrariedades na construção de $H$ são descritas por uma arbitrária matriz simpléctica $\Omega_{\gamma \delta}^{(0)}$ e as funções arbitrárias $\varphi(t, x)$ e $c(t)$.

Podemos ver que existe uma família de Lagrangianas (2.46) que levam às mesmas equações de movimento (2.32). É fácil ver que as acções com a mesma $\Omega_{\gamma \delta}^{(0)}$ mas diferentes funções $\varphi(t, x)$ e $c(t)$ diferem por uma derivada total (que chamamos de uma diferença trivial). Uma diferença nas funções de Lagrange relacionadas com a escolha de diferentes matrizes simplécticas $\Omega_{\alpha \beta}^{(0)}$ não é trivial. As correspondentes Lagrangianas são conhecidas como as Lagrangianas $s$-equivalentes.

Como exemplo, vamos considerar uma teoria com as equações de movimento de forma ${ }^{2}$

$$
\dot{x}=A(t) x+j(t) .
$$

Princípio de ação para esta teoria pode ser construído seguindo a abordagem descrita acima.

A solução do problema de Cauchy (2.53) é

$$
x(t)=\Gamma(t) x_{(0)}+\gamma(t),
$$

onde a matriz $\Gamma(t)$ é uma solução fundamental de (2.53), ou seja,

$$
\dot{\Gamma}=A \Gamma, \Gamma(0)=1,
$$

e $\gamma(t)$ é uma solução parcial de (2.53). Usando (2.44), construimos a matriz $\Omega^{3}$,

$$
\Omega=\Lambda^{T} \Omega^{(0)} \Lambda, \quad \Lambda=\Gamma^{-1} .
$$

e encontramos as funções $J$ e $H$ de acordo com (2.51) e (2.52),

$$
J=\frac{1}{2} x \Omega, H=\frac{1}{2} x B x-C x,
$$

onde

$$
B=\frac{1}{2}\left(\Omega A-A^{T} \Omega\right), C=\Omega j .
$$

Assim, a ação para a teoria geral quadrática é

$$
S[x]=\frac{1}{2} \int d t(x \Omega \dot{x}-x B x-2 C x) .
$$

Em conclusão, observamos que sempre é possível construir uma ação Lagrangiana para qualquer sistema de equações não-Lagrangianas, em um espaço de configurações extendido, seguindo uma ideia simples, proposto pela primeira vez por Bateman [22]. Tal Lagrangiana

\footnotetext{
${ }^{2}$ Aqui usamos as notações de matrizes, $x=\left(x^{\alpha}\right), A(t)=\left(A(t)_{\beta}^{\alpha}\right), j(t)=\left(j(t)^{\alpha}\right), \alpha, \beta=1, . ., 2 n$.

${ }^{3}$ Para simplicidade vamos escolher a matriz $\Omega^{(0)}$ sendo uma matriz constante, não-singular e antisimétrica.
} 
tem a forma de uma soma de equações de movimento iniciais multiplicados por multiplicadores de Lagrange correspondentes, que se tratam como as novas variáveis. As equações de EulerLagrange para esse tipo de ação além de conter as equações de movimento iniciais tem ainda novas equações de movimento para os multiplicadores de Lagrange. O uso desta abordagem tem algumas dificuldades já no nível clássico, porque precisa se fornecer uma interpretação para as novas variáveis . Dificuldades adicionais (as métricas indefinidas) podem aparecer após a quantização [23]-[26]. 


\section{Capítulo 3}

\section{Descrição Lagrangiana da carga elétrica com radiação}

\subsection{Introdução}

Uma peculiaridade notável da eletrodinâmica clássica de partículas pontuais é a possibilidade de uma análise coerente de backreaction de radiação em termos da equação de movimento local que não contêm campos dinâmicos. Esta equação é conhecida como as equação de LorentzDirac. A diferença entre ela e a equação ordinária de Lorentz consiste em termos adicionais com a derivada de terceira ordem em relação ao tempo da trajetória [71], [47], [76]. A presença da derivada de terceira ordem em relação ao tempo implica em duas consequências imediatas. Primeiro, porque a dinâmica de uma partícula já não é reversível no tempo, e este fato está de acordo com o conceito intuitivo da irreversibilidade física de um processo da radiação. Segundo, os dados iniciais para esta equação tem que incluir não somente a posição e a velocidade iniciais da partícula, como custuma ser na mecânica clássica ordinária, mas também a aceleração inicial. A última circunstância leva ao fato de que, juntamente com as soluções físicas, as equações de Lorentz-Dirac contêm as soluções que não admitem uma interpretação razoável física (por exemplo, soluções auto-aceleradas [47], [76]). Além disso, em contraste da equação de Lorentz, a equação de Lorentz-Dirac é uma equação não-Lagrangiana.

É importante observar que, tanto no nível clássico como no quântico, a eletrodinâmica de Maxwell é uma teoria essencialmente perturbativa, onde os cálculos são feitos apenas sob a forma de séries assimtóticas pela constante de acoplamento (a carga elétrica $e$ ). No caso geral, essas séries são divergentes, mas o cálculo dos primeiros termos da série da expansão normalmente fornece uma boa aproximação dos dados experimentais, tendo em vista o fato que a constante estrutural é muito pequena. Em nível clássico, uma abordagem coerente perturbativa da eletrodinâmica leva à seguinte regra da seleção: as soluções físicas da equação de Lorentz-Dirac são apenas aquelas que tenham um limite suave do desligamento da interação, ou seja, quando a carga da partícula tende a zero. Acontece que todas soluções físicas podem 
ser descritas por uma equação de segunda ordem, que se chama a equação reduzida de LorentzDirac.

Neste capítulo, vamos investigar a possibilidade de construir uma formulação Lagrangiana para a equação de Lorentz-Dirac clássica e reduzida.

\subsection{Impossibilidade de uma descrição Lagrangiana do sistema clássico de Lorentz-Dirac}

Nesta seção vamos considerar a questão da existência de funcional da ação para a equação relativística de Lorentz - Dirac [47, 76]

$$
g_{\mu}=-m \ddot{x}_{\mu}+\frac{e}{c} F_{\mu \nu} \dot{x}^{\nu}+\frac{2 e^{2}}{3 c^{3}}\left(\dddot{x}_{\mu}-\frac{1}{c^{2}} \dot{x}_{\mu} \ddot{x}_{\nu} \ddot{x}^{\nu}\right)=0,
$$

que descreve a dinâmica efetiva da carga pontual com a backreaction da radiação. Aqui, $x^{\mu}=$ $(t, \mathrm{x})$ são as coordenadas da partícula no espaço-tempo quadri-dimensional; $F_{\mu \nu}=(\mathbf{E}, \mathbf{H})$ é o tensor do campo electromagnético; as constantes $c$ e $e$ são a velocidade da luz e a carga elétrica, respectivamente.

Como já foi mencionado, (3.1) é uma equação não-Lagrangiana. Porém, podemos procurar o multiplicador da integração. Vamos supor que (3.1) admita a existência do multiplicador da integração $h_{\nu}^{\mu}(t, x, \dot{x} \ldots)$, det $h_{\nu}^{\mu} \neq 0$, ou seja, que existe uma ação $S[x]$ cuja variação leva ao sistema

$$
h_{\mu}^{\nu} g_{\nu}=0
$$

Como as equações (3.1) não incluem as derivadas mais altas do que a de terceira ordem, a Lagrangiana correspondente depende apenas das derivadas de primeira e de segunda ordem da trajetória, além disso, a dependência das derivadas de segunda ordem deve ser linear. Isto implica:

$$
L=a(x, \dot{x})+b_{\mu}(x, \dot{x}) \ddot{x}^{\mu} .
$$

Comparando as equações de Euler-Lagrange desta Lagrangiana com as equações (3.2), concluímos que

$$
\begin{aligned}
& \frac{\partial b_{\mu}}{\partial \dot{x}^{\nu}}-\frac{\partial b_{\nu}}{\partial \dot{x}^{\mu}}=\gamma h_{\mu \nu}, \\
& c^{2}\left(\frac{\partial^{2} b_{\nu}}{\partial \dot{x}^{\mu} \partial \dot{x}^{\lambda}}-\frac{\partial^{2} b_{\mu}}{\partial \dot{x}^{\nu} \partial \dot{x}^{\lambda}}\right) \ddot{x}^{\nu} \ddot{x}^{\lambda}=-\gamma h_{\mu \lambda} \dot{x}^{\lambda} \ddot{x}_{\nu} \ddot{x}^{\nu},
\end{aligned}
$$

com $\gamma=2 e^{2} / 3 c^{3}$. Em particular, é óbvio que a matriz $h_{\mu \nu}=h_{\mu}^{\lambda} \eta_{\lambda \nu}\left(\eta_{\mu \nu}\right.$ é o tensor métrico de Minkowsky) deve ser anti-simétrica. Derivando a primeira das equações (3.4) em relação a $\dot{x}^{\lambda}$, e substituindo o resultado na segunda equação, obtemos

$$
\frac{\partial h_{\mu \sigma}}{\partial \dot{x}^{\lambda}} \ddot{x}^{\sigma} \ddot{x}^{\lambda}=-\frac{1}{c^{2}} h_{\mu \alpha} \dot{x}^{\alpha} \ddot{x}_{\nu} \ddot{x}^{\nu}
$$




\section{CAPÍTULO 3. DESCRIÇÃO LAGRANGIANA DA CARGA ELÉTRICA COM RADIAÇÃO33}

Como $h_{\mu \nu}$ não depende de $\ddot{x}^{\mu}$, a equação acima leva a condição

$$
\frac{\partial h_{\mu \nu}}{\partial \dot{x}^{\lambda}}=-\frac{1}{c^{2}} h_{\mu \alpha} \dot{x}^{\alpha} \eta_{\nu \lambda} .
$$

Já que $h_{\mu \nu}$ é anti-simétrico, chegamos à relação

$$
h_{\mu \alpha} \dot{x}^{\alpha} \eta_{\nu \lambda}=-h_{\nu \alpha} \dot{x}^{\alpha} \eta_{\mu \lambda},
$$

cuja contração com $\eta^{\nu \lambda}$ leva a

$$
h_{\mu \nu} \dot{x}^{\nu}=0 .
$$

Levando em consideração (3.7) obtemos de (3.6) que

$$
\frac{\partial h_{\mu \nu}}{\partial \dot{x}^{\lambda}}=0 .
$$

Isso significa que $h_{\mu \nu}$ não depende de $\dot{x}^{\lambda}$. Então de (3.7) concluímos que a matriz $h_{\mu \nu}$ é singular. Assim, chegamos numa contradição, o multiplicador da integração não pode ser singular. A equação clássice de Lorentz-Dirac (3.1) não admite a existência do multiplicador da integração.

Para a equação não-relativística de Lorentz-Dirac

$$
\mathrm{g}=-m \ddot{\mathrm{x}}+e \mathbf{E}+\frac{e}{c}[\dot{\mathrm{x}}, \mathbf{H}]+\frac{2 e^{2}}{3 c^{3}} \dddot{\mathbf{x}}=0 .
$$

os mesmos argumentos que acima mostram que o multiplicador de integração deverá ser antisimétrico, e isto, mais uma vez, entra em contradição com a condição da não-singularidade, uma vez que (3.8) é um sistema de três equações e, portanto, o multiplicador de integração deve ser uma matriz de terceira ordem, no entanto, qualquer matriz anti-simétrica de rank ímpar é necessariamente singular.

Além do problema com o princípio variacional a presença do termo adicional com derivada de terceira ordem leva a algumas dificuldades com a interpretação física. Em primeiro lugar, de acordo com os postulados da mecânica clássica, o estado de sistema mecânico deve ser unicamente determinado pela atribuição de sua posição e velocidade iniciais. Estes dados iniciais são manifestamente insuficientes para atribuir o estado do sistema descrito por equações de terceira ordem. Em segundo lugar, como demonstra uma análise simples, as equações (3.1) e (3.8) junto com as soluções fisicamente razoáveis, admitem as soluções não-físicas (por exemplo, auto-aceleradas) $[47,76]$.

Os problemas mencionados são relacionadas e têm uma solução comum. Ou seja, postula-se que somente aquelas soluções da equação de Lorentz-Dirac são trajetórias físicas, que tenham um limite suave quando a carga da partícula tende a zero. Essa exigência elimina as soluções patológicas, além disso, cada trajetória física pode ser determinada através da atribuição da posição e a velocidade inicial de uma partícula. O significado da condição imposta torna-se evidente quando observa-se que a derivada de terceira ordem entra na equação de Lorentz-Dirac 
sendo multiplicado por perturbação $e^{2}$. Portanto, no limite do desligamento da interação $(e \rightarrow$ 0) a ordem da equação de Lorentz-Dirac efetivamente reduz a dois, depois, equação descreve o movimento da partícula livre. Em outras palavras, no âmbito de uma abordagem perturbativa da interação eletromagnética, quando a constante do acoplamento e é considerada pequena, a presença de um termo com derivada de terceira ordem é tratada não como o aparecimento de novos graus de liberdade da partícula (que seria um absurdo do ponto de vista físico), mas apenas como uma pequena deformação da dinâmica da partícula livre ${ }^{1}$.

Em vez de extrair um por um todas as soluções físicas da equação da terceira ordem de Lorentz-Dirac, podemos impor o problema da busca de tal equação da segunda ordem que descreve todas essas. As soluções desta equação devem obedecer as equações iniciais de LorentzDirac e ser automaticamente suave (em e). Esta equação se chama a equação reduzida de Lorentz-Dirac e o procedimento da construção dela é a perturbativa redução de ordem.

\subsection{O sistema das equações fisicamente equivalentes}

\subsubsection{Perturbativa redução de ordem na equação de Lorentz-Dirac}

Vamos supor que existe tal equação de segunda ordem,

$$
\ddot{x}^{i}=f^{i}(x, \dot{x}, e), \quad i=1,2,3,
$$

que todas as soluções desta equação $x^{i}(t, e)$ (a) são funções suaves em $e$, e (b) obedecem a equação de Lorentz-Dirac não-relativística (3.8). O cumprimento de condição (a) pode ser garantido se exigir que o lado direito da equação (3.9) é uma função analítica da $e$. Vamos examinar a condição (b). Derivando a equação (3.9) em relação ao tempo obtemos

$$
\dddot{x}^{i}=\frac{\partial f^{i}}{\partial x^{j}} \dot{x}^{j}+\frac{\partial f^{i}}{\partial \dot{x}^{j}} \ddot{x}^{j}
$$

Usando a equação (3.9) de novo, temos

$$
\dddot{x}^{i}=\frac{\partial f^{i}}{\partial x^{j}} \dot{x}^{j}+\frac{\partial f^{i}}{\partial \dot{x}^{j}} f^{j}
$$

Assumindo agora que todas as soluções da (3.9) também obedecem a equação (3.8) e, em seguida, expressando na última equação $\dddot{x}^{i}$ e $\ddot{x}^{i}$ através $\dot{x}^{i}$ e $x^{i}$, com ajuda de (3.9), (3.10), obtemos uma identidade:

$$
m f^{i}=e E^{i}+\frac{e}{c}[\dot{x}, H]^{i}+\frac{2 e^{2}}{3 c^{3}}\left(\frac{\partial f^{i}}{\partial x^{j}} \dot{x}^{j}+\frac{\partial f^{i}}{\partial \dot{x}^{j}} f^{j}\right) .
$$

\footnotetext{
${ }^{1}$ Ao mesmo tempo, o valor da carga $e$, que faz o papel do parâmetro da deformação, pode ser qualquer. Tudo que se exige é a existência de um limite suave das soluções quando $e \rightarrow 0$.
} 


\section{CAPÍTULO 3. DESCRIÇÃO LAGRANGIANA DA CARGA ELÉTRICA COM RADIAÇÃO35}

Esta identidade é a equação para a definição da função $f^{i}(x, \dot{x})$. A solução dela pode ser encontrada na forma de uma série de potência $e$ :

$$
f^{i}=\sum_{k=0}^{\infty} e^{k} f_{(k)}^{i}
$$

onde

$$
\begin{aligned}
& f_{(0)}^{i}=0, \\
& f_{(1)}^{i}=(m)^{-1} E^{i}+(m c)^{-1}[\dot{x}, H]^{i}, \\
& f_{(k)}^{i}=\frac{2}{3 m c^{3}}\left(\frac{\partial f_{(k-2)}^{i}}{\partial x^{j}} \dot{x}^{j}+\sum_{l=0}^{k-2} \frac{\partial f_{(k-2-l)}^{i}}{\partial \dot{x}^{j}} f_{(l)}^{j}\right), \quad k \geq 2 .
\end{aligned}
$$

Assim, obtivemos uma equação de segunda ordem que descreve a dinâmica efetiva da partícula carregada num campo eletromagnético com uma força backreaction da radiação. Todas as soluções desta equação também são as soluções da equação de Lorentz-Dirac e, além disso, estão suaves em $e$.

Para certas configurações simples dos campos externos, a expressão para a força $f^{i}$ pode ser encontrado em uma forma simples. Por exemplo, no caso de um campo magnético homogêneo, $\mathbf{H}=(0,0, H), \mathbf{E}=0$, a equação de Lorentz-Dirac reduzida tem a forma

$$
\begin{aligned}
& \ddot{x}=\alpha \dot{x}-\beta \dot{y}, \\
& \ddot{y}=\beta \dot{x}+\alpha \dot{y}, \\
& \ddot{z}=0,
\end{aligned}
$$

onde, no sistema de unidades $m=c=1$, temos

$$
\alpha=\frac{6-\sqrt{6} \sqrt{3+\sqrt{9+64 e^{6} H^{2}}}}{8 e^{2}} \approx-\frac{2}{3} e^{4} H^{2}, \quad \beta=\frac{e H \sqrt{6}}{\sqrt{3+\sqrt{9+64 e^{6} H^{2}}}} \approx e H .
$$

Caso colocar $\alpha=0$ nas equações (3.13), elas se tornam as equações de Lorentz ordinárias descrevendo a dinâmica de uma partícula carregada no campo magnético "efetivo" $\mathbf{B}=(0,0, \beta / e)$. Para $\alpha \neq 0$ as trajetórias da partícula são espirais no plano $x y$, a partícula cai no centro. Por este motivo o parâmetro $\alpha$ pode ser identificado com o coeficiente de atrito da radiação.

\subsubsection{A construção da Lagrangiana}

Agora vamos considerar o problema da construção da função de Lagrange que leva às equações (3.13). Já que o movimento ao longo do eixo $z$ é livre, é suficiente examinar o sistema de duas primeiras equações:

$$
\begin{aligned}
& \ddot{x}=\alpha \dot{x}-\beta \dot{y}, \\
& \ddot{y}=\beta \dot{x}+\alpha \dot{y},
\end{aligned}
$$




\section{CAPÍTULO 3. DESCRIÇÃO LAGRANGIANA DA CARGA ELÉTRICA COM RADIAÇÃO36}

Neste caso a matriz $B$ definida no primeiro capítulo é:

$$
B_{k}^{i}=\frac{1}{2}\left(\begin{array}{cc}
\alpha^{2}-\beta^{2} & -2 \alpha \beta \\
2 \alpha \beta & \alpha^{2}-\beta^{2}
\end{array}\right)
$$

e condição (2.23) implica que

$$
\operatorname{Tr}\left(h_{i j}\right)=h_{11}+h_{22}=0 .
$$

Agora, é fácil ver que a solução geral de equações (2.9), (2.15), (2.17), (2.18), e (2.23) é determinada por uma função arbitrária $\phi(z, w)$ e tem a forma

$$
h_{i j}=\left(\begin{array}{cc}
F+\bar{F} & i(F-\bar{F}) \\
i(F-\bar{F}) & -(F+\bar{F})
\end{array}\right) .
$$

Onde, $F=\phi\left(\dot{\xi} e^{-\gamma t}, \dot{\xi}-\gamma \xi\right) e^{-\gamma t}, \xi=x+i y, \gamma=\alpha+i \beta$, e barra significa a conjugação complexa.

Escolhendo $\phi=1 / z$, vamos obter a solução mais simples independente do tempo:

$$
h_{i j}=\frac{2}{\dot{x}^{2}+\dot{y}^{2}}\left(\begin{array}{cc}
\dot{x} & \dot{y} \\
\dot{y} & -\dot{x}
\end{array}\right) \text {. }
$$

Usando a formula (2.10), encontramos a seguinte Lagrangeana:

$$
L=\frac{1}{2} \dot{x} \ln \left(\dot{x}^{2}+\dot{y}^{2}\right)+\dot{y} \operatorname{arctg}\left(\frac{\dot{x}}{\dot{y}}\right)+\alpha x-\beta y .
$$

As equações correspondentes de Euler-Lagrange

$$
\frac{\ddot{x} \dot{x}+\ddot{y} \dot{y}}{\dot{x}^{2}+\dot{y}^{2}}=\alpha, \quad \frac{\ddot{x} \dot{y}-\ddot{y} \dot{x}}{\dot{x}^{2}+\dot{y}^{2}}=\beta,
$$

são, obviamente, equivalente às equações iniciais (3.15) com excepção do ponto $\dot{x}=\dot{y}=0$.

Assim, podemos ver que formalmente existe a solução do problema da construção de uma Lagrangiana para a partícula carregada no campo magnético uniforme com backreaction da radiação. No entanto, temos que notar que de acordo com o tratamento perturbativo da interação electromagnética, exigimos que no limite $e \rightarrow 0$ (ou seja, $\alpha \rightarrow 0, \beta \rightarrow 0$ ) a Lagrangiana $L$ deve se transformar na Lagrangiana de uma partícula livre,

$$
L_{0}=\frac{1}{2}\left(\dot{x}^{2}+\dot{y}^{2}\right)
$$

pelo modulo da derivada total. Infelizmente, nem a Lagrangeana (3.19) nem qualquer outra Lagrangeana construída a partir da matriz (3.17) não têm um correto limite livre. Porque, de acordo com (3.16), o traço da matriz de Hesse da qualquer Lagrangeana para o sistema de equações (3.15) deve ser igual a zero, e essa propriedade permanece válida após o limite considerado. Por outro lado, o traço da matriz de Hesse da Lagrangiana $L_{0}$ é igual 2. Esta contradição prova a afirmação.

Contudo, a ausência de uma satisfatória descrição física de backreaction da radiação no formalismo de segunda ordem deixa a esperança que tal descrição é possível no formalismo de primeira ordem. 


\subsection{Funcional de ação no formalismo de primeira ordem}

Vamos considerar o problema da construção da funcional da ação no formalismo de primeira ordem para o sistema físico que no formalismo de segunda ordem se descreve por equações de movimento (3.15). Para isso, elas serãm substituidas por um sistema equivalente de equações de primeira ordem. Vamos escolher as variáveis auxiliares como

$$
p=\dot{x}+\frac{\beta}{2} y, \quad q=\dot{y}-\frac{\beta}{2} x .
$$

Como resultado, chegamos ao sistema

$$
\begin{aligned}
& \dot{x}=p-\frac{\beta}{2} y, \\
& \dot{p}=-\frac{\beta}{2} q-\frac{\beta^{2}}{4} x+\alpha\left(p-\frac{\beta}{2} y\right), \\
& \dot{y}=q+\frac{\beta}{2} x, \\
& \dot{q}=\frac{\beta}{2} p-\frac{\beta^{2}}{4} y+\alpha\left(q+\frac{\beta}{2} x\right) .
\end{aligned}
$$

A ação quadrática para este sistema tem a forma

$$
\begin{aligned}
& S[x]=\int d t \frac{e^{-\alpha t}}{4\left(\alpha^{2}+\beta^{2}\right)}[2 a(t)(p \dot{x}-x \dot{p}+q \dot{y}-y \dot{q})+ \\
& +2 b(t)(q \dot{x}-x \dot{q}+y \dot{p}-p \dot{y})+2 c(t)(p \dot{q}-q \dot{p})+2 d(t)(x \dot{y}-y \dot{x})+ \\
& \left.+e(t)\left(p^{2}+q^{2}\right)+f(t)\left(x^{2}+y^{2}\right)+g(t)(p x+q y)+j(t)(q x-p y)\right],
\end{aligned}
$$

com

$$
\begin{gathered}
a(t)=\alpha^{2} \cos (\beta t)+\frac{1}{2} \beta^{2}\left(e^{-\alpha t}+e^{\alpha t}\right), \quad b(t)=\alpha^{2} \sin (B t)-\alpha \beta e^{\alpha t}+\alpha \beta \cos (\beta t), \\
c(t)=e^{-\alpha t} \beta+2 \alpha \sin (\beta t), \quad d(t)=\frac{1}{4} \beta^{3}\left(e^{-\alpha t}-e^{\alpha t}\right)-\frac{1}{2} \beta^{2} \alpha \sin (\beta t)+\alpha^{2} \beta\left(\cos (\beta t)-e^{\alpha t}\right), \\
e(t)=e^{-\alpha t} \beta^{2}+\alpha^{2} \cos (\beta t)+\alpha \sin (\beta t) \beta, \\
f(t)=\frac{1}{4} \beta\left(\beta^{3} e^{-\alpha t}+\beta \alpha^{2} \cos (\beta t)-\alpha \sin (\beta t)\left[\beta^{2}+2 \alpha^{2}\right]\right), \\
g(t)=-\alpha \cos (\beta t) \beta^{2}-\alpha^{3} \cos (\beta t), \quad j(t)=-\alpha^{3} \sin (\beta t)+e^{-\alpha t} \beta^{3}+\alpha^{2} \beta \cos (\beta t) .
\end{gathered}
$$

No limite do desaparecimento do atrito $(\alpha \rightarrow 0)$, a ação (3.23) se transforma para a ação Hamiltoniana canônica da partícula carregada no campo magnético homogêneo. Assim, foi construída uma ação fisicamente razoável da partícula carregada no campo magnético uniforme com backreaction da radiação. A quantização canônica desse modelo será considerada no capítulo seguinte. 


\section{Capítulo 4}

\section{Quantização canônica de sistemas não-Lagrangianos}

\subsection{Introdução}

Neste capítulo consideramos a abordagem para construir as teorias quânticas que no limite clássico reproduzem as equações não-Lagrangianas para os valores médios. De fato consideramos a quantização canônica de teorias Lagrangianas com vínculos dependentes do tempo que são relacionados com as teoria não Lagrangianas. Demostramos na seção 2 que a formulação Hamiltoniana de Lagrangianas de primeira ordem que correspondem as teorias nãoLagrangianas leva a teoria Hamiltoniana com vínculos de segunda classe dependentes do tempo. Assim mostramos que sistemas não-Lagrangianos são de fato equivalentes aos sistemas Lagrangianos no formalismo de primeira ordem, mas com vínculos dependentes do tempo na formulação Hamiltoniana. A quantização canónica de tais teorias é um problema não trivial e foi considerado no caso geral no livro [49]. Na seção 3 nós descrevemos o procedimento geral do livro [49] e adotamos ele para nosso caso. Na seção 4 aplicamos a abordagem geral para construir a quantização de sistemas físicos com as equações arbitrárias lineares de movimento. Depois consideramos a quantização canônica do oscilador harmônico amortecido (sec. 6) e da carga elétrica com radiação (sec.7).

\subsection{Formulação Hamiltoniana}

Nesta seção vamos construir a formulação Hamiltoniana para teoria com Lagrangiana da primeira ordem (2.46). Para isso, seguimos as considerações gerais do livro [49]. Em primeiro lugar construimos ação $S^{v}[x, \pi, v]$, que, neste caso, tem a forma

$$
S^{v}[x, \pi, v]=\int\left[J_{\alpha}(t, x) v^{\alpha}-H(t, x)+\pi_{\alpha}\left(\dot{x}^{\alpha}-v^{\alpha}\right)\right] d t
$$


que depende das momentas $\pi_{\alpha}$ conjugadas para as coordenadas $x^{\alpha}$, bem como das velocidades $v^{\alpha}$. As equações

$$
\frac{\delta S^{v}}{\delta v^{\alpha}}=\Phi_{\alpha}(t, x, \pi)=\pi_{\alpha}-J_{\alpha}(t, x)=0
$$

não permitem expressar as velocidades como funções das $x$ e $\pi$, que implica a aparência dos vínculos primários $\Phi_{\alpha}(t, x, \pi)$, e as velocidades $v^{\alpha}$ se tornam multiplicadores de Lagrange para estes vínculos, assim que a ação (4.1) se torna ação Hamiltoniana da teoria com vínculos primários (4.2),

$$
S_{\mathrm{H}}=\int d t\left\{\pi_{\alpha} \dot{x}^{\alpha}-H^{(1)}\right\}, H^{(1)}=H(t, x)+\lambda^{\alpha} \Phi_{\alpha}(t, x, \pi),
$$

com as equações de movimento

$$
\dot{\eta}=\left\{\eta, H^{(1)}\right\}, \Phi=0
$$

onde $\eta=(x, \pi)$.

Os vínculos primários são vínculos de segunda classe, porque em virtude da condição que o multiplicador de integração $\Omega_{\alpha \beta}(t, x)$ é uma matriz não singular, temos:

$$
\left\{\Phi_{\alpha}, \Phi_{\beta}\right\}=\Omega_{\alpha \beta}(t, x) \Longrightarrow \operatorname{det}\left\{\Phi_{\alpha}, \Phi_{\beta}\right\} \neq 0 \text {. }
$$

Assim, os vínculos secundários não aparecem e todos os multiplicadores de Lagrange $\lambda$ podem ser determinados da condição da consistência para os vínculos primários:

$$
\begin{aligned}
\dot{\Phi}_{\alpha} & =\partial_{t} \Phi_{\alpha}+\left\{\Phi_{\alpha}, H^{(1)}\right\}=0 \Longrightarrow-\partial_{t} J_{\alpha}-\partial_{\alpha} H+\lambda^{\beta}\left\{\Phi_{\alpha}, \Phi_{\beta}\right\}=0 \Longrightarrow \\
\lambda^{\beta} & =\omega^{\beta \alpha}\left(\partial_{t} J_{\alpha}+\partial_{\alpha} H\right), \omega^{\beta \alpha}=\Omega_{\beta \alpha}^{-1} .
\end{aligned}
$$

Usando os multiplicadores de Lagrange (4.6) nas equações (4.4), podemos escrever essas equações na forma

$$
\dot{\eta}=\{\eta, H\}_{D(\Phi)}+\left\{\eta, \Phi_{\alpha}\right\} \omega^{\alpha \beta} \partial_{t} J_{\beta}, \Phi=0,
$$

onde $\{\cdot, \cdot\}_{D(\Phi)}$ são parênteses de Dirac em respeito aos vínculos da segunda classe $\Phi$. Para as variáveis canônicas as parênteses de Dirac são

$$
\begin{aligned}
& \left\{x^{\alpha}, x^{\beta}\right\}_{D(\Phi)}=\omega^{\alpha \beta}, \\
& \left\{\pi_{\alpha}, \pi_{\beta}\right\}_{D(\Phi)}=\partial_{\alpha} J_{\rho} \omega^{\rho \gamma} \partial_{\beta} J_{\gamma}, \\
& \left\{x^{\alpha}, \pi_{\beta}\right\}_{D(\Phi)}=\delta_{\beta}^{\alpha}+\omega^{\alpha \gamma} \partial_{\beta} J_{\gamma} .
\end{aligned}
$$

Se formalmente introduzir o momento $\epsilon$ conjugo ao tempo $t$, e definir os parênteses de Poisson no espaço extendido das variáveis canônicas $(x, \pi ; t, \epsilon)=(\eta ; t, \epsilon)$, veja [49], podemos reescrever (4.7) na forma seguinte:

$$
\dot{\eta}=\{\eta, H+\epsilon\}_{D(\Phi)}, \Phi=0
$$


As equações (4.9) representem a formulação Hamiltoniana dos sistemas não-Lagrangianos com as equações de movimento de primeira ordem (2.4). Note que os vínculos Hamiltonianos nesta formulação são de segunda classe e dependem explicitamente do tempo. A quantização canônica de tais teorias pode ser feita de acordo com as linhas gerais do [49]. Abaixo nós apresentamos os detalhes desta quantização e depois a aplicamos para o nosso caso.

\subsection{Quantização canônica}

Para a teoria Hamiltoniana com os vínculos de segunda classe $\Phi_{l}(\eta, t)$ dependentes do tempo o procedimento da quantização no quadro de Schrödinger é realizado da seguinte forma. As variaveis do espaço de fase $\eta$ são substituídos por operadores $\hat{\eta}(t)$ sujeitos as relações de commutação e equações dos seguintes vínculos:

$$
\left[\hat{\eta}^{A}(t), \hat{\eta}^{B}(t)\right]=\left.i\left\{\eta^{A}, \eta^{B}\right\}_{D(\Phi)}\right|_{\eta=\hat{\eta}}, \Phi_{l}(\hat{\eta}(t), t)=0
$$

A evolução temporal deles é postulada como (aqui não discutimos sobre o ordenamento dos operadodes, para as detalhes veja [68])

$$
\frac{d}{d t} \hat{\eta}(t)=\left.\{\eta, \epsilon\}_{D(\Phi)}\right|_{\eta=\hat{\eta}}=-\left.\left\{\eta, \Phi_{l}\right\}\{\Phi, \Phi\}_{l l^{\prime}}^{-1} \partial_{t} \Phi_{l^{\prime}}\right|_{\eta=\hat{\eta}}
$$

Para cada grandeza física $F$, dada na formulação Hamiltoniana pela função $F(t, \eta)$, colocamos em correspondência o operador de Schrödinger $\hat{F}(t)$, pela regra $\hat{F}(t)=F(t, \hat{\eta}(t))$. Para os dois arbitrários operadores de Schrödinger $\hat{F}(t)$ e $\hat{G}(t)$, a relação

$$
[\hat{F}(t), \hat{G}(t)]=\left.i\{F, G\}_{D(\Phi)}\right|_{\eta=\hat{\eta}}
$$

é obedecida como consequênccia da (4.10). Os estados quânticos do sistema são descritos por vetores $\Psi$ do espaço de Hilbert com o produto escalar $\left(\Psi, \Psi^{\prime}\right)$. A evoluçao do vetor $\Psi$ é determinada por equação de Schrödinger

$$
i \frac{\partial \Psi(t)}{\partial t}=\hat{H} \Psi(t)
$$

onde o Hamiltoniano quântico $\hat{H}$ é construído de acordo com a função clássica $H(t, \eta)$ como $\hat{H}(t)=H(t, \hat{\eta}(t))$. Os valores médios $\langle F\rangle_{t}$ da grandeza física $F$ são determinados como valores médios do correspondente operador de Schrödinger $\hat{F}(t)=F(t, \hat{\eta}(t))$ em respeito ao vetor do espaço de Hilbert $\Psi(t)$,

$$
\langle F\rangle_{t}=(\Psi(t), \hat{F}(t) \Psi(t))
$$

Como $\hat{H}$ é um operador auto-adjunto, a evolução em tempo dos vetores do estado $\Psi(t)$ é unitária,

$$
\Psi(t)=U(t) \Psi(0), U^{+}(t)=U^{-1}(t)
$$


onde $U(t)$ é um operador da evolução.

No quadro de Heisenberg, onde os vetores do estado são "congelados" e a evolução em tempo é determinada por causa dos operadores de Heisenberg $\check{\eta}(t)=U^{-1}(t) \hat{\eta}(t) U(t)$, pode-se ver [49] que

$$
\begin{aligned}
& \frac{d}{d t} \check{\eta}=\left.\{\eta, H(t, \eta)+\epsilon\}_{D(\Phi)}\right|_{\eta=\check{\eta}}, \\
& {\left[\check{\eta}^{A}(t), \check{\eta}^{B}(t)\right]=\left.i\left\{\eta^{A}, \eta^{B}\right\}_{D(\Phi)}\right|_{\eta=\check{\eta}}, \quad \Phi(\check{\eta}(t), t)=0,}
\end{aligned}
$$

enquanto para operadores de Heisenberg $\check{F}(t)=U^{-1}(t) \hat{F}(t) U(t)=F(t, \check{\eta}(t))$, temos

$$
\frac{d}{d t} \check{F}(t)=\left.\{F(t, \eta), H(t, \eta)+\epsilon\}_{D(\Phi)}\right|_{\eta=\check{\eta}}
$$

ou seja

$$
\frac{d}{d t} \check{F}(t)=-i[\check{F}(t), \check{H}(t)]+\left.\{F(t, \eta), \epsilon\}_{D(\Phi)}\right|_{\eta=\check{\eta}} .
$$

Os valores médios $\langle F\rangle_{t}$ no quadro de Heisenber de acordo com (4.14) e (4.15) são determinados como

$$
\langle F\rangle_{t}=(\Psi(0), \check{F}(t) \Psi(0)) .
$$

A quantização descrita obedece o princípio de correspondência já que as equações quânticas (4.16) têm a mesma forma que as clássicas (4.9).

Note que no caso geral a dependência do tempo dos operadores de Heisenberg nas teorias consideradas não é unitária. Em outras palavras, não existe nenhum operador "Hamiltoniano" cujo comutador com o operador de Heisenberg daria a derivada total do tempo deste operador. Este fato pode ser explicado pela existência de dois fatores que determinam a evolução do operador de Heisenberg. O primeiro fator é a evolução unitária do vetor do estado no quadro de Schrödinger, enquanto segundo é a própria dependência do tempo do operador de Schrödinger $\hat{\eta}$, que em geral não tem um caráter unitário. A existência destes dois fatores está relacionada com a divisão da parte direita de (4.18) em dois termos. Físicamente isto pode ser explicado pelo fato que a dinâmica se desenvolve na superfície dos vínculos que depende do tempo. E essa dependência em geral não é unitária.

Abaixo nós aplicamos o esquema geral da quantização para sistemas em consideração. Tendo em conta os parênteses de Dirac (4.8), podemos escrever as relações de comutação (4.10) para operadores do espaço de fase

$$
\begin{aligned}
& {\left[\hat{x}^{\alpha}, \hat{x}^{\beta}\right]=\left.i \omega^{\alpha \beta}\right|_{x=\hat{x}},} \\
& {\left[\hat{\pi}_{\alpha}, \hat{\pi}_{\beta}\right]=\left.i \partial_{\alpha} J_{\rho} \omega^{\rho \gamma} \partial_{\beta} J_{\gamma}\right|_{x=\hat{x}},} \\
& {\left[\hat{x}^{\alpha}, \hat{\pi}_{\beta}\right]=i \delta_{\beta}^{\alpha}+\left.i \omega^{\alpha \gamma} \partial_{\beta} J_{\gamma}\right|_{x=\hat{x}} .}
\end{aligned}
$$

No caso em consideração o Hamiltoniano clássico $H$ não depende das momentas $\pi_{\alpha}$, e por isso, para determinar o Hamiltoniano quântico $\hat{H}$, precisamos saber a dependência do tempo 
somente dos operadores $\hat{x}^{\alpha}$. Da equação (4.11) temos

$$
\frac{d}{d t} \hat{x}^{\alpha}=\left.\omega^{\alpha \beta}(t, x) \partial_{t} J_{\beta}(t, x)\right|_{x=\hat{x}}
$$

\subsection{Quantização de teorias quadráticas gerais}

A teoria quântica de sistemas quadráticos é um problema amplamente discutido em física, que tem uma série de aplicações importantes (veja por exemplo, [78]-[82]). Porém, os artigos mencionados consideram apenas casos de sistemas quadráticos "Hamiltonianos" com as equações de movimento na forma $\dot{x}^{\alpha}=\left\{x^{\alpha}, H\right\}_{\text {can }}$, onde $H$ é uma Hamiltoniana quadrática e $\{\cdot, \cdot\}_{\text {can }}$ é um parêntese de Poisson canónica. Nós consideramos as teorias descritas por arbitrárias lineares equações de movimento na forma (2.53): $\dot{x}=A(t) x+j(t)$. No segundo capítulo mostrou-se que tais sistemas também admitem a existênsia da ação quadrática, mas os parênteses de Poisson já não vão ser mais canônicos e no caso geral podem depender do tempo. Chamaremos tais sistemas de teorias quadráticas gerais. Nesta seção consideramos a quantização canônica de teorias quadráticas gerais.

Para teorias quadráticas gerais as condições (4.20), (4.21) se tornam

$$
\begin{aligned}
& {\left[\hat{x}^{\alpha}, \hat{x}^{\beta}\right]=i \omega^{\alpha \beta}(t),} \\
& \frac{d}{d t} \hat{x}^{\alpha}=-\frac{1}{2} \omega^{\alpha \beta}(t) \dot{\Omega}_{\beta \gamma}(t) \hat{x}^{\gamma} .
\end{aligned}
$$

A dependência do tempo dos operadores $\hat{x}$ pode ser facilmente encontrada:

$$
\hat{x}^{\alpha}(t)=\Phi^{\alpha \beta}(t) \hat{x}_{0}^{\beta} .
$$

Aqui a matriz $\Phi$ obedece a equação

$$
\dot{\Phi}=-\frac{1}{2} \omega \dot{\Omega} \Phi, \quad \Phi(0)=E,
$$

e os operadores $\hat{x}_{0}$ obedecem as relações de comutação seguintes:

$$
\left[\hat{x}_{0}^{\alpha}, \hat{x}_{0}^{\beta}\right]=i\left(\Omega_{\alpha \beta}^{(0)}\right)^{-1}=i\left(\begin{array}{cc}
\mathbf{0} & \mathbf{I} \\
-\mathbf{I} & \mathbf{0}
\end{array}\right)
$$

veja capítulo 2. É conveniente dividir os operadores $\hat{x}_{0}^{\alpha}$ em operadores das coordenadas próprias e momentas correspondentes $\hat{x}_{0}^{\alpha}=\left(\hat{q}^{i}, \hat{p}_{i}\right), \alpha=1, . ., 2 n, i=1, . ., n$. Os operadores $\hat{q}$ e $\hat{p}$ obedecem as relações de comutação canônicas

$$
\left[\hat{q}^{i}, \hat{p}_{j}\right]=i \delta_{j}^{i},\left[\hat{q}^{i}, \hat{q}^{j}\right]=\left[\hat{p}_{i}, \hat{p}_{j}\right]=0 .
$$


O Hamiltoniano quântico em (4.13) se torna

$$
\hat{H}=\frac{1}{2} \hat{x}_{0} \Phi^{T} B \Phi \hat{x}_{0}-C \Phi \hat{x}_{0},
$$

onde a matriz $B$ é determinada em (2.58).

A quantização acima é equivalente à quantização em coordenadas de Darboux. A transformação $x \rightarrow \Phi(t) x_{0}$ é uma passagem para as coordenadas de Darboux $x_{0}$, porque (4.25) implica

$$
\Phi^{t} \Omega \Phi=\Omega_{0} .
$$

Então os parênteses de Poisson entre as coordenadas $x_{0}$ são canônicas. Portanto, $\Phi=\Gamma(t) R(t)$, onde $\Gamma(t)$ é uma solução fundamental do sistema (2.53). Porém, ao contrário da teoria clássica, agora a matriz $R(t)$ é fixa, obedecendo as seguintes condições

$$
\dot{R}=\Omega_{0} \Gamma^{t} B \Gamma R, \quad R(0)=E .
$$

Assim, pode-se também reescrever a Hamiltoniana em (4.28) como:

$$
\hat{H}=-\frac{1}{2} \hat{x}_{0} R^{T} \Omega^{(0)} \dot{R} \hat{x}_{0}+C \Gamma R \hat{x}_{0}
$$

É notável que caso a matriz $A$ que determina as equações (2.53) é uma constante, a matriz que determina a parte quadrática da Hamiltoniana quântica (4.28) também é constante e é igual

$$
\Phi^{T} B \Phi=B(0)=\frac{1}{2}\left(\Omega^{(0)} A-A^{T} \Omega^{(0)}\right) .
$$

Este fato é fácil para ver porque o derivado em relação ao tempo desta matriz é egual a zero, por causa das equações (4.25), (2.56) e (2.58):

$$
\frac{d}{d t}\left(\Phi^{T} B \Phi\right)=0
$$

Assim, neste caso a matriz $\Phi$ pode ser determinada das equações algebráicas (4.29) e (4.32).

Note que se começar com o sistemas quadráticos "Hamiltonianos" com paréntese de Poisson canônica, a quantização descrita coencide com a quantização canónica usual, porque neste caso a equação (4.23) se torna $d \hat{x} / d t=0$, i.e., $\hat{x}(t)=\hat{x}_{0}$.

No quadro de Heisenberg as equações (4.16) para os operadores $\check{x}$ têm a forma

$$
\begin{gathered}
\frac{d}{d t} \check{x}=A(t) \check{x}+j(t), \\
{\left[\check{x}^{\alpha}, \check{x}^{\beta}\right]=i \omega^{\alpha \beta}(t) .}
\end{gathered}
$$


As equações (4.33) coincidem (o princípio da correspondência) com as equações de movimento clássicas (2.53); porém, as relações de comutação (4.34) são diferentes do caso canónico. Então a evolução dos operadores $\check{x}$ pode ser escrita como

$$
\check{x}(t)=\Gamma(t) \check{x}_{0}+\gamma(t),
$$

onde os operadores $\check{x}_{0}$ bem como $\hat{x}_{0}$ obedecem as relações de comutação canónicas

$$
\left[\check{x}_{0}^{\alpha}, \check{x}_{0}^{\beta}\right]=i\left(\Omega_{\alpha \beta}^{(0)}\right)^{-1}=i\left(\begin{array}{cc}
\mathbf{0} & \mathbf{I} \\
-\mathbf{I} & \mathbf{0}
\end{array}\right) .
$$

Assim, os valores médios $\langle F\rangle_{t}$ das grandezas físicas $F$ de acordo com (4.19) são determinadas como os valores médios dos operadores correspondentes $\check{F}(t)=F(t, \check{x}(t))$ com respeito aos vetores do estado $\Psi(0)$ iniciais, ou seja,

$$
\langle F\rangle_{t}=\left(\Psi(0), F\left(t, \Gamma(t) \check{x}_{0}+\gamma(t)\right) \Psi(0)\right) .
$$

Podemos ver que a evolução quântica das grandezas físicas nos sistemas quadráticos gerais é completamente determinada pela evolução clássica.

\subsection{Quantização do oscilador harmônico amortecido}

O método proposto da quantização canônica de sistemas não-Lagrangianos e, em particular, de teorias quadráticas gerais pode ser ilustrado no exemplo do oscilador harmônico amortecido. Este problema atrai atenção há mais de 50 anos, existem diferentes abordagens para a sua solução, mas todas elas apresentam pontos fracos, veja por exemplo [28]-[27], [3].

A equação do movimento clássico do oscilador harmônico amortecido é uma equação nãoLagrangiana e tem a forma

$$
\ddot{r}+2 \alpha \dot{r}+\omega^{2} r=0,
$$

onde $\omega$ é a frequência angular e $\alpha \geq 0$ é o coeficiente do atrito. Apresentando uma variável auxiliar $y=\dot{r}$, reduzimos (4.38) para o sistema equivalente de duas equações de primeira ordem:

$$
\dot{r}=y, \dot{y}=-\omega^{2} r-2 \alpha y .
$$

Seguindo o método geral do capítulo 2, construimos a ação $S$ que fornece (4.39) como as equações de Euler-Lagrange,

$$
S=\frac{1}{2} \int d t\left[y \dot{r}-r \dot{y}-\left(y^{2}+2 \alpha r y+\omega^{2} r^{2}\right)\right] e^{2 \alpha t} .
$$

Note que a equação (4.38) pode ser representada como

$$
\frac{d}{d t}\left(e^{2 \alpha t} \dot{r}\right)+e^{2 \alpha t} \omega^{2} r=0
$$


ou seja, como a equação Lagrangiana com massa e frequência dependentes do tempo. Neste caso a massa $e^{2 \alpha t}$ é o multiplicador de integração para a equação (4.38); mas, como já foi mencionado no capítulo 2, no caso geral o multiplicador de integração nem sempre existe.

Agora aplicamos para o modelo construído a quantização canônica descrita na seção anterior. As relações de comutação (4.22) e as equações (4.23) que determinam a evolução dos operadores de "Schrödinger" $\hat{r}$ e $\hat{y}$ são

$$
\begin{aligned}
{[\hat{r}, \hat{y}] } & =i e^{-2 \alpha t},[\hat{r}, \hat{r}]=[\hat{y}, \hat{y}]=0, \\
\frac{d}{d t} \hat{r} & =-\alpha \hat{r}, \quad \frac{d}{d t} \hat{y}=-\alpha \hat{y} .
\end{aligned}
$$

A solução delas tem a forma

$$
\hat{r}=e^{-\alpha t} \hat{q}, \quad \hat{y}=e^{-\alpha t} \hat{p},
$$

onde os operadores $\hat{q}$ e $\hat{p}$ obedecem as relações de comutação canônicas

$$
[\hat{q}, \hat{p}]=i,[\hat{q}, \hat{q}]=[\hat{p}, \hat{p}]=0 .
$$

De acordo com (4.28), o Hamiltoniano quântico correspondente é

$$
\hat{H}=\frac{1}{2}\left[\hat{p}^{2}+\alpha(\hat{q} \hat{p}+\hat{p} \hat{q})+\omega^{2} \hat{q}^{2}\right] .
$$

E pode ser modificado na forma

$$
\hat{H}=\frac{1}{2}\left[\hat{P}^{2}+\left(\omega^{2}-\alpha^{2}\right) \hat{Q}^{2}\right]
$$

com ajuda da transformação canônica $(\hat{p}, \hat{q}) \rightarrow(\hat{P}, \hat{Q})$, onde $\hat{P}=\hat{p}+\alpha \hat{q}$, e $\hat{Q}=\hat{q}$. A correspondente função da geração é $W=q P-\alpha q^{2} / 2$.

Como sempre, definimos a energia clássica do sistema na forma

$$
E=\frac{1}{2}\left(\dot{r}^{2}+\omega^{2} r^{2}\right)=\frac{1}{2}\left(y^{2}+\omega^{2} r^{2}\right) .
$$

É facil ver que ela depende do tempo como: $E=E_{0} e^{-2 \alpha t}$. Usando (4.43), obtemos a expressão para o operador $\hat{E}$ que corresponde a grandeza clássica $E$,

$$
\hat{E}=\frac{1}{2} e^{-2 \alpha t}\left[\hat{P}^{2}-\alpha(\hat{P} \hat{Q}+\hat{Q} \hat{P})+\left(\omega^{2}+\alpha^{2}\right) \hat{Q}^{2}\right] .
$$

Consideramos primeiro o caso, $\alpha<\omega$. Assim (4.44) é o Hamiltoniano do oscilador harmônico com a frequencia angular $\tilde{\omega}=\sqrt{\omega^{2}-\alpha^{2}}$. Os estados estacionares da equação de Schrödinger correspondente têm a forma

$$
\begin{aligned}
& \Psi_{n}=e^{-i E_{n} t} \psi_{n}(Q), E_{n}=\tilde{\omega}\left(n+\frac{1}{2}\right), n=0,1, \ldots \\
& \psi_{n}(Q)=\frac{1}{\sqrt{2^{n} n !}}\left(\frac{\tilde{\omega}}{\pi}\right)^{1 / 4} e^{-\tilde{\omega} Q^{2} / 2} H_{n}(Q \sqrt{\tilde{\omega}})
\end{aligned}
$$


Os valores médios da energia (operador (4.45)) em tais estados podem ser facilmente calculados,

$$
\langle E\rangle_{n}=\left(n+\frac{1}{2}\right) \frac{\omega^{2}}{\tilde{\omega}} e^{-2 \alpha t} .
$$

Em cada instante do tempo fixo, o espectro energético é discreto, porém, ele cai com o tempo exatamente como na teoria clássica. A mesma conclusão foi obtida em [29]-[23] onde como o ponto inicial foi adotada a ação no formalismo de segunda ordem construída usando o método do multiplicador de integração.

Os casos quando $\alpha \geq \omega$ correspondem ao movimento aperiódico na teoria clássica [72]. Sua interpretação quântica não é muito clara por causa do caráter contínuo do espectro do Hamiltoniano.

A generalização não trivial da equação (4.38) podia ser oscilador amortecido $n$-dimensional ${ }^{1}$

$$
\ddot{r}+2 \mathbf{a} \dot{r}+\omega r=0,
$$

com matrizes a e $\omega$ constantes e simétricos. Introduzindo as variáveis auxiliares, $y=\dot{r}, y=\left(y^{i}\right)$, reduzimos (4.48) para a seguintes equações de primeira ordem:

$$
\begin{aligned}
& \dot{x}=A x,\left(x=\left(x^{\alpha}\right)=\left(r^{i}, y^{i}\right)\right), \\
& A=\left(\begin{array}{cc}
0 & \mathbf{I} \\
-\omega & -2 \mathbf{a}
\end{array}\right) .
\end{aligned}
$$

Isso é um conjunto de equações lineares com coeficientes constantes. Neste caso, o Hamiltoniano quântico correspondente pode ser construída, de acordo com a (4.32), como:

$$
\hat{H}=\frac{1}{2} \hat{x}_{0} B(0) \hat{x}_{0},
$$

onde

$$
B(0)=\left(\begin{array}{cc}
\omega & \mathbf{a} \\
\mathbf{a} & \mathbf{I}
\end{array}\right)
$$

e os operadores $\hat{x}_{0}$ são divididos em operadores das coordenadas $\hat{q}$ próprias e momentas correspondentes $\hat{p}$ com as as relações de comutação canônicas (6.50). Depois, para a construção da solução do problema quântico com o Hamiltoniano quadrático (4.50) pode-se seguir por exemplo, os artigos [78, 79].

\subsection{Quantização da carga elétrica com radiação}

O sistema das equações de primeira ordem (3.22) que descreve o movimento da partícula carregada no campo magnético uniforme com backreaction da radiação, é um sistema linear com

\footnotetext{
${ }^{1}$ Usamos as notações de matriz, $r=\left(r^{i}\right), \mathbf{a}=\left(a_{j}^{i}\right), \omega=\left(\omega_{j}^{i}\right), i, j=1, . ., n$.
} 
coeficientes constantes. Para tal caso, o Hamiltoniano quântico correspondente pode ser construído de acordo com a (4.32) como

$$
\hat{H}=\frac{1}{2}\left[\hat{p}_{1}^{2}+\hat{p}_{2}^{2}+\frac{\alpha}{2}\left(\hat{p}_{1} \hat{q}_{1}+\hat{q}_{1} \hat{p}_{1}+\hat{p}_{2} \hat{q}_{2}+\hat{q}_{2} \hat{p}_{2}\right)+\beta\left(\hat{p}_{2} \hat{q}_{1}-\hat{p}_{1} \hat{q}_{2}\right)+\frac{\beta^{2}}{4}\left(\hat{q}_{1}^{2}+\hat{q}_{2}^{2}\right)\right]
$$

com operadores $\hat{q}_{i}$ e $\hat{p}_{j}$ obedecendo as relações de comutação canônicas

$$
\left[\hat{q}_{i}, \hat{p}_{j}\right]=i,\left[\hat{q}_{i}, \hat{q}_{j}\right]=\left[\hat{p}_{i}, \hat{p}_{j}\right]=0, \quad i, j=1,2 .
$$

Com a ajuda da transformação canônica $\left(\hat{p}_{1}, \hat{q}_{1} ; \hat{p}_{2}, \hat{q}_{2}\right) \rightarrow(\hat{p}, \hat{x} ; \hat{q}, \hat{y})$, onde

$$
\hat{p}=\hat{p}_{1}+\frac{\alpha}{2} \hat{q}_{1}, \hat{x}=\hat{q}_{1}, \hat{q}=\hat{p}_{2}+\frac{\alpha}{2} \hat{q}_{2}, \hat{y}=\hat{q}_{2},
$$

reescrevemos (4.51) na forma

$$
\hat{H}=\frac{1}{2}\left[\hat{p}^{2}+\hat{q}^{2}+\beta(\hat{q} \hat{x}-\hat{p} \hat{y})+\frac{\beta^{2}-\alpha^{2}}{4}\left(\hat{x}^{2}+\hat{y}^{2}\right)\right] .
$$

Condição que backreaction da radiação é uma deformação pequena da dinâmica da partícula carregada no caso considerado implica $\alpha \ll \beta$, por isso $\alpha^{2}$ será omitida em (4.52).

Consideramos estados próprios $\Psi$ para dois operadores que comutam mutuamente $\hat{H}$ e $\hat{L}=\hat{p} \hat{y}-\hat{q} \hat{x}$

$$
\hat{H} \Psi=E \Psi, \hat{L} \Psi=M \Psi .
$$

É conveniente fazer a transformação canónica $(\hat{p}, \hat{x} ; \hat{q}, \hat{y}) \rightarrow(\hat{P}, \hat{X} ; \hat{Q}, \hat{Y})$,

$$
\begin{aligned}
& \hat{P}=\hat{p}-\frac{\beta}{2} \hat{y}, \hat{X}=\frac{1}{\beta}\left(\hat{q}+\frac{\beta}{2} \hat{x}\right), \\
& \hat{Q}=\hat{q}-\frac{\beta}{2} \hat{x}, \hat{Y}=\frac{1}{\beta}\left(\hat{p}+\frac{\beta}{2} \hat{y}\right) .
\end{aligned}
$$

É facil ver que

$$
\hat{H}=\frac{1}{2}\left(\hat{P}^{2}+\beta^{2} \hat{X}^{2}\right), \hat{L}=\beta^{-1}\left(\hat{H}_{1}-\hat{H}\right), \hat{H}_{1}=\frac{1}{2}\left(\hat{Q}^{2}+\beta^{2} \hat{Y}^{2}\right) .
$$

Operadores $\hat{H}$ e $\hat{H}_{1}$ são Hamiltonianas de dois osciladores harmônicos independentes. A solução do problema de autovalores (4.53) é,

$$
\Psi=\Psi_{n, l}(X, Y)=\psi_{n}(X) \psi_{l}(Y), \quad E_{n}=\beta\left(n+\frac{1}{2}\right), M_{n l}=l-n, n, l=0,1,2, \ldots
$$

com $\psi_{n}$ e $\psi_{l}$ são estados próprios das Hamiltonianos $\hat{H}$ e $\hat{H}_{1}$ (dados por exemplo por (4.46)). Enfim, as soluções $\Psi(t)$ da equação de Schrödinger com o Hamiltoniano $\hat{H}$ tem a forma

$$
\Psi(X, Y, t)=e^{-i E_{n} t} \Psi_{n, l}(X, Y) .
$$


Definimos a energia clássica $E$ do sistema em consideração de acordo com [72] como a energia mecânica do sistema sem atrito,

$$
E=\frac{1}{2}\left[p^{2}+q^{2}+\beta(q x-p y)+\frac{\beta^{2}}{4}\left(x^{2}+y^{2}\right)\right] .
$$

Pode se ver que ela depende do tempo como: $E=E_{0} e^{-2 \alpha t}$. O operador $\hat{E}$ que corresponde a $E$ é:

$$
\hat{E}=\frac{1}{2}\left[\hat{P}^{2}+\beta^{2} \hat{X}^{2}+\alpha(\hat{X} \hat{Y}-\hat{P} \hat{Q})\right] e^{-2 \alpha t}-2 \alpha\left(\frac{\alpha}{\beta}\right)[\hat{P} \hat{Y}+\hat{Q} \hat{X}] e^{-2 \alpha t}+o\left(\frac{\alpha}{\beta}\right) .
$$

Os valores médios deste operador nos estados (4.54) são

$$
\langle E\rangle_{n l}=\beta\left(n+\frac{1}{2}\right) e^{-2 \alpha t} .
$$

Como no caso do oscilador harmônico amortecido em cada instante do tempo fixo, o espectro energético é discreto, porém, ele cai com o tempo exatamente como na teoria clássica.

\subsection{Conclusão}

É importante notar que qualquer sistema não-degenerado das equações diferenciais sendo escrito na forma equivalente das equações de primeira ordem pode ser obtido a partir de princípio da ação mínima. No caso geral, tal sistema não fornece bastante informação para fixar um classe das teorias quânticas que no seu limite clássico reproduzem essas equações diferenciais para os valores médios. Portanto, as considerações físicas adicionais tem que ser usadas para a escolha da teoria quântica adequada. Em particular, se as equações não-Lagrangeanos descrevem um sistema dissipativo, em que a dissipação se trata como o resultado da interação do sistema mecânico com ambiente (reservatório), é razoavel considerar o sistema e o reservatório como dois subsistemas interagidos de um sistema fechado. Depois a descrição quântica de sistema dissipativo pode ser obtida da teoria quântica de sistema completo calculando a média (integração) sobre os estados do reservatório. Esta abordagem foi usada em muitos trabalhos: [32]-[46]. Porém, ela não pode ser considerada como uma quantização do sistema dissipativo inicial porque a teoria quântica foi construida para um outro sistema físico - sistema fechado, composto de reservatório e de sistema mecânico. Nesta tese consideramos uma abordagem, em que construimos a teoria quântica para o sistema físico com as equações de movimento determinadas. O comportamento "não-Lagrangiano" deste sistema aparece na teoria através do campo externo dependente do tempo, que entra na teoria atravéz dos vínculos na formulação Hamiltoniana. Isto é uma situação física principalmente diferente da dissipação do subsistema. Portanto, as teorias quânticas obtidas do nosso procedimento podem ser úteis para descrever as propriedades quânticas tanto de sistemas dissipativos, quanto de sistemas não-Lagrangianos da outra natureza física, como monopólo magnético. 


\section{Capítulo 5}

\section{Integral de trajetória na MQNC}

\subsection{Introdução}

Recentemente as teorias quânticas de campo no espaço-tempo não comutativo que recentemente recebeu bastante atenção, veja por exemplo [50] e outras referências nesses artigos. No caso mais simples o espaço-tempo não-comutativo $d+1$ dimensional pode ser realizado pelos operadores de coordenada $\hat{q}^{\mu}, \mu=0,1, \ldots, d$, que obedecem as seguintes relações de comutação

$$
\left[\hat{q}^{\mu}, \hat{q}^{\nu}\right]=i \theta^{\mu \nu}
$$

$\operatorname{com} \theta^{\mu \nu}$ é uma matriz anti-simétrica constante das valores reais. Obviamente, muitos problemas principais ligados com não-comutatividade podem ser examinados já em mecânica quântica nãocomutativa (MQNC). Alguns trabalhos nessa direção consideram a generalização dos modelos quânticos bem conhecidos (oscilador harmônico [55], a partícula carregada no campo magnético [58], o átomo de Hydrogênio [59], a partícula no campo de Aharonov-Bohm [60, 61] e sistema no potencial central [62]) para o caso não comutativo, tentando extrair as possíveis observáveis diferenças com o caso comutativo. Outros trabalhos consideram adaptações para o caso não comutativo de abordagens e tecnicas gerais na mecânica quântica. Assim a abordagem da integral de trajetória na MQNC não-relativística foi estudada nos artigos [63]-[67] e considerados os exemplos mais simples do oscilador harmônico [64] e da partícula livre [67].

Observe que ações clássicas de teorias de campo no espaço-tempo não-comutativo podem ser escritos como ações clássicas modificadas mas já no espaço-tempo comutativo (introduzindo o produto estrela). Depois a quantização de tais ações modificadas (chamamos eles de ações $\theta$-modificados) reproduzirá tanto não-comutatividade do espaço-tempo como características quânticas da teoria de campo correspondente. Considerando a mecânica quântica de uma partícula (ou o sistema de $N$ partículas) com coordenadas não-comutativas, pergunta-se como construir a ação clássica $\theta$-modificada para este sistema de dimensão finito. Como no caso da teoria de campo tal ação $\theta$-modificada depois da quantização deve reproduzir como nãocomutatividade de coordenadas tanto características quânticas do sistema físico correspondente. 
Para a mecânica quântica não-relativística o problema mencionado foi resolvido em [84]. Neste capítulo consideramos a questão da construção de ações $\theta$-modificados para MQNC relativística. Serão construídas tais ações para partículas relativísticas com e sem spin. A idéia chave é extrair ações $\theta$-modificadas de partículas relativisticas da representação via integral de trajetória dos propagadores da correspondente teoria do campo não-comutativa. Consideramos equações de Klein-Gordon e de Dirac para propagadores casuais em tais teorias. Depois construímos para estes propagadores a representação via integral de trajetória usando técnicas desenvolvidas em $[74,83]$ para o caso comutativo. Nós tratamos as ações efetivas nesta representação como ações $\theta$-modificadoas de partículas relativísticas. Para confirmar a interpretação nós construímos a quantização canônica de ações obtidas. Assim obtivemos as equações de Klein-Gordon e de Dirac na teoria de campo não-comutativa. A ação $\theta$-modificada da partícula relativística com spin é uma generalização da ação pseudoclássica da superpartícula de Berezin-Marinov [68] para caso não-comutativo.

\subsection{Representações via integral de trajetória dos propa- gadores da partícula em teoria quântica de campo não-comutativa}

\subsubsection{O caso sem spin}

Em teoria de campo os efeitos da não-comutatividade do espaço-tempo podem ser realizados pela substituição do produto usual das funções pelo produto estrela:

$$
\begin{aligned}
f(x) \star g(x) & =f(x) \exp \left\{\frac{i}{2} \overleftarrow{\partial}_{\mu} \theta^{\mu \nu} \overrightarrow{\partial_{\nu}}\right\} g(x) \\
& =f\left(x^{\mu}+\frac{i}{2} \theta^{\mu \nu} \partial_{\nu}\right) g(x),
\end{aligned}
$$

com $f(x)$ e $g(x)$ são funções arbitrárias das variáveis comutativas $x^{\mu}$.

A ação da teoria não-comutativa do campo escalar $\Phi$ que interage com o campo externo eletromagnético do fundo $A_{\mu}(x)$ é

$$
S_{\text {scal-field }}^{\theta}=\int d^{D} x\left[\left(P_{\mu} \star \Phi\right) \star\left(P^{\mu} \star \bar{\Phi}\right)+m^{2} \Phi \bar{\Phi}\right], P_{\mu}=i \partial_{\mu}-g A_{\mu}(x) .
$$

As equação de Euler-Lagrange correspondente

$$
\frac{\delta S_{\text {scal-field }}^{\theta}}{\delta \bar{\Phi}}=0 \Rightarrow\left[P_{\mu} \star P^{\mu}-m^{2}\right] \star \Phi=0,
$$


com ajuda de (5.2) pode ser reescrita na forma

$$
\begin{aligned}
& \left(\tilde{P}^{2}-m^{2}\right) \Phi=0, \quad \tilde{P}^{2}=\tilde{P}_{\mu} \tilde{P}^{\mu}, \\
& \tilde{P}_{\mu}=i \partial_{\mu}-g A_{\mu}\left(x^{\mu}+\frac{i}{2} \theta^{\mu \nu} \partial_{\nu}\right),
\end{aligned}
$$

e é um análogo da equação de Klein-Gordon no caso não-comutativo. O propagador em teoria não-comutativa de campo escalar é uma função de Green causal $D^{c}(x, y)$ da equação (5.5),

$$
\left(\tilde{P}^{2}-m^{2}\right) D^{c}(x, y)=-\delta(x-y)
$$

Partindo desse ponto vamos seguir o procedimento elaborado em [74] para construir a representação via integral de trajetória deste propagador. Consideramos $D^{c}(x, y)$ o elemento de matriz do operador $\hat{D}^{c}$ no espaço de Hilbert

$$
D^{c}(x, y)=\left\langle x\left|\hat{D}^{c}\right| y\right\rangle .
$$

Aqui $|x\rangle$ são autovetores dos operadores que comutam mutualmente $\hat{x}^{\mu}$,

$$
\hat{x}^{\mu}=\hat{q}^{\mu}+\frac{1}{2 \hbar} \theta^{\mu \nu} \hat{p}_{\nu}
$$

onde os operadores $\hat{q}^{\mu}$ obedecem as relações de comutação (5.1) e $\hat{p}_{\mu}$ são operadores da momenta conjugados aos $\hat{x}^{\mu}$,

$$
\begin{aligned}
& {\left[\hat{x}^{\mu}, \hat{p}_{\nu}\right]=i \hbar \delta_{\nu}^{\mu},\left[\hat{x}^{\mu}, \hat{x}^{\nu}\right]=\left[\hat{p}_{\mu}, \hat{p}_{\nu}\right]=0} \\
& \hat{x}^{\mu}|x\rangle=x^{\mu}|x\rangle,\left\langle x\left|y>=\delta^{D}(x-y), \quad \int\right| x><x\right| d x=I .
\end{aligned}
$$

A equação (5.7) implica $\hat{D}^{c}=\left(m^{2}-\Pi^{2}\right)^{-1}$, onde ${ }^{1}$

$$
\begin{aligned}
& \hat{\Pi}_{\mu}=-\hat{p}_{\mu}-g A_{\mu}(\hat{q}), \quad\left[\hat{\Pi}_{\mu}, \hat{\Pi}_{\nu}\right]=-i g \hat{F}_{\mu \nu}, \\
& \hat{F}_{\mu \nu}=\partial_{\mu} A_{\nu}(\hat{q})-\partial_{\nu} A_{\mu}(\hat{q})+i g\left[A_{\mu}(\hat{q}), A_{\nu}(\hat{q})\right] .
\end{aligned}
$$

Por causa da propriedade do produto estrela $f(\hat{q}) g(\hat{q})=(f \star g)(\hat{q})$, podemos representar o operador $\hat{F}_{\mu \nu}$ na forma seguinte

$$
\hat{F}_{\mu \nu}=F_{\mu \nu}^{\star}(\hat{q}), \quad F_{\mu \nu}^{\star}(q)=\partial_{\mu} A_{\nu}-\partial_{\nu} A_{\mu}+i g\left(A_{\mu} \star A_{\nu}-A_{\nu} \star A_{\mu}\right) .
$$

\footnotetext{
${ }^{1}$ Usamos notação $\Pi^{2}=\Pi_{\mu} \Pi^{\mu}$.
} 
Usando a representação do tempo próprio de Schwinger para o operador inverso, obtemos:

$$
\begin{aligned}
& D^{c}=D^{c}\left(x_{\text {out }}, x_{\text {in }}\right)=i \int_{0}^{\infty}\left\langle x_{\text {out }}\left|\exp \left[-\frac{i}{\hbar} \hat{\mathcal{H}}(\lambda)\right]\right| x_{\text {in }}\right\rangle d \lambda, \\
& \hat{\mathcal{H}}(\lambda)=\lambda\left(m^{2}-\Pi^{2}\right) .
\end{aligned}
$$

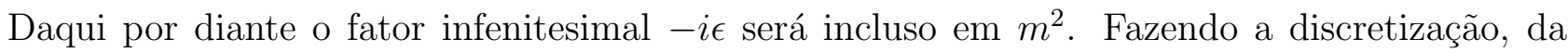
mesma maneira como em [74], obtemos a representação via integral de trajetória do propagador $(5.13)$

$$
D^{c}=i \int_{0}^{\infty} d \lambda_{0} \int_{x_{\text {in }}}^{x_{\text {out }}} D x \int_{\lambda_{0}} D \lambda \int D p D \pi \exp \left\{\frac{i}{\hbar}\left[S_{\text {scal-part }}^{\theta}+S_{\mathrm{GF}}\right]\right\}
$$

onde

$$
\begin{aligned}
& S_{\text {scal-part }}^{\theta}=\int_{0}^{1}\left[\lambda\left(\mathcal{P}^{2}-m^{2}\right)+p_{\mu} \dot{x}^{\mu}\right] d \tau, S_{\mathrm{GF}}=\int_{0}^{1} \pi \dot{\lambda} d \tau, \\
& \mathcal{P}_{\mu}=-p_{\mu}-g A_{\mu}\left(x^{\mu}-\frac{1}{2 \hbar} \theta^{\mu \nu} p_{\nu}\right), \dot{x}=\frac{d x}{d \tau}, \dot{\lambda}=\frac{d \lambda}{d \tau} .
\end{aligned}
$$

A integração funcional em (5.14) vai sobre trajetórias $x^{\mu}(\tau), p_{\mu}(\tau), \lambda(\tau)$, e $\pi(\tau)$, parametrizadas por um parâmetro invariante $\tau \in[0,1]$ e obedecem as condições de fronteira $x(0)=x_{\text {in }}, x(1)=x_{\text {out }}, \lambda(0)=\lambda_{0}$.

Como as momentas entram nos argumentos do potencial eletromagnético $A_{\mu}$, a integração sobre as momentas na representação (5.14) é complicado para fazer no caso geral. Por outro lado, podemos passar das coordenadas $x$ para novas variaveis $q$,

$$
q^{\mu}=x^{\mu}-\frac{1}{2 \hbar} \theta^{\mu \nu} p_{\nu}
$$

que correspondem de certa forma aos operadores não-comutativos $\hat{q}^{\mu}$ (5.1). Então

$$
\begin{aligned}
& D^{c}=i \int_{0}^{\infty} d \lambda_{0} D p \int_{x_{i n}-\theta p / 2 \hbar}^{x_{\text {out }}-\theta p / 2 \hbar} D q \int_{\lambda_{0}} D \lambda \int D \pi \exp \left\{\frac{i}{\hbar} S_{\text {scal-part }}^{\theta}+S_{\mathrm{GF}}\right\}, \\
& S_{\text {scal-part }}^{\theta}=\int_{0}^{1}\left\{\lambda\left[\left(p_{\mu}+g A_{\mu}(q)\right)^{2}-m^{2}\right]+p_{\mu} \dot{q}^{\mu}-\frac{1}{2 \hbar} \dot{p}_{\mu} \theta^{\mu \nu} p_{\nu}\right\} d \tau .
\end{aligned}
$$

Assim fugimos da dificuldade mencionada acima, mas uma outra dificuldade aparece. A ação $S_{\text {scal }}^{\theta}$ em (5.17) inclui um termo "inconveniente" $\dot{p}_{\mu} \theta^{\mu \nu} p_{\nu} / 2 \hbar$. Por causa desse termo a possibilidade de integrar sobre as momentas é relacionada com a estrutura da matriz $\theta^{\mu \nu}$ e pode ser realizada passando para as coordenadas de Darboux. 
Podemos tratar a representação (5.17) como integral de trajetória Hamiltoniana para propagador de partícula escalar na teoria de campo não-comutativa. $\mathrm{O}$ argumento da exponente na integral (5.17) pode ser considerado como a ação efetiva Hamiltoniana da partícula escalar no espaço-tempo não-comutativo. Ele consiste de duas partes: a primeira $S_{\mathrm{GF}}$ pode ser tratada como o termo da fixação de gauge e corresponde, de fato, a condição de gauge $\dot{\lambda}=0$. A parte restante da ação efetiva $S_{\text {scal-part }}^{\theta}$ pode ser tratada como $\theta$-modificação da ação Hamiltoniana usual da partícula sem spin relativística no caso comutativo. A diferença entre esta ação e a ação comutativa correspondente (veja [74]) é no termo adicional $\dot{p}_{\mu} \theta^{\mu \nu} p_{\nu} / 2 \hbar$.

\subsubsection{Partícula spinorial}

Consideramos a ação $\theta$-modificada da teoria não-comutativa do campo espinorial $\Psi$ que interage com o campo externo eletromagnético do fundo $A_{\mu}$. Sendo escrita no espaço comutativo de Minkowski $D$-dimensional com as coordenadas $x^{\mu}, \mu=0,1, \ldots, D-1$, esta ação tem a forma

$$
S_{\text {spinor-field }}^{\theta}=\int d x^{D} \bar{\Psi} \star\left(P_{\mu} \gamma^{\mu}+m\right) \star \Psi
$$

onde $\gamma^{\mu}$ são matrizes gama em $D$ dimenções, $\left[\gamma^{\mu}, \gamma^{\nu}\right]_{+}=2 \eta^{\mu \nu}$. Nós consideramos $D$ sendo par $D=2 d$, para simplicedade e como a generalização do 4-dim. espaço de Minkowski, o caso impar pode ser considerado da mesma maneira, seguindo trabalho [83]. É sabido que [86] quando o número das dimenções é par, a representação via matrizes da álgebra de Clifford com dimensionalidade $\operatorname{dim} \gamma^{\mu}=2^{d}$ sempre existe. Em outras palavras $\gamma^{\mu}$ são matrizes $2^{d} \times 2^{d}$. Nesta dimenção pode-se introduzir uma outra matriz $\gamma^{D+1}=r \gamma^{0} \gamma^{1} \ldots \gamma^{D-1}$, com $r=1$, se $d$ é par, e $r=i$, se $d$ é ímpar, que anticomuta com todas $\gamma^{\mu}$ (um análogo da $\gamma^{5}$ em quatro dimenções), $\left[\gamma^{D+1}, \gamma^{\mu}\right]_{+}=0,\left(\gamma^{D+1}\right)^{2}=-1$. As equações de Euler-Lagrange

$$
\frac{\delta S_{\text {spinor-field }}^{\theta}}{\delta \bar{\Psi}}=0 \Rightarrow\left(P_{\mu} \gamma^{\mu}+m\right) \star \Psi=0,
$$

com ajuda de (5.2) podem ser reescritas na forma

$$
\left(\tilde{P}_{\mu} \gamma^{\mu}-m\right) \Psi=0, \tilde{P}_{\mu}=i \partial_{\mu}-g A_{\mu}\left(x^{\mu}+\frac{i}{2} \theta^{\mu \nu} \partial_{\nu}\right)
$$

e representam o análogo da equação de Dirac no caso não-comutativo. O propagador em teoria não-comutativa de campo escalar é uma função de Green causal $G^{c}(x, y)$ da equação (5.20),

$$
\left(\tilde{P}_{\mu} \gamma^{\mu}-m\right) G^{c}(x, y)=-\delta^{D}(x-y) .
$$

Seguindo $[74,83]$, passamos para o operador de Dirac $\theta$-modificado que é homogêneo em matrizes $\gamma$. Na verdade, vamos reescrever a equação $(5.21)$ em termos do propagador $\tilde{G}^{c}(x, y)$ transformado por $\gamma^{D+1}$,

$$
\tilde{G}^{c}(x, y)=G^{c}(x, y) \gamma^{D+1}, \quad\left(\tilde{P}_{\mu} \tilde{\gamma}^{\mu}-m \gamma^{D+1}\right) \tilde{G}^{c}(x, y)=\delta^{D}(x-y),
$$


onde $\tilde{\gamma}^{\mu}=\gamma^{D+1} \gamma^{\mu}$. As matrizes $\tilde{\gamma}^{\mu}$ têm as mesmas relações de comutação como as iniciais sem tilda $\left[\tilde{\gamma}^{\mu}, \tilde{\gamma}^{\nu}\right]_{+}=2 \eta^{\mu \nu}$, e anticomutam com a matriz $\gamma^{D+1}$. O conjunto de $D+1$ matrizes gama $\tilde{\gamma}^{\nu}$ e $\gamma^{D+1}$ formam a representação da álgebra de Clifford em $2 d+1$ dimenções. Vamos identificar essas matrizes através de $\Gamma^{n}$,

$$
\begin{aligned}
& \Gamma^{n}= \begin{cases}\tilde{\gamma}^{\mu}, & n=\mu=0, \ldots, D-1 \\
\gamma^{D+1}, & n=D\end{cases} \\
& {\left[\Gamma^{k}, \Gamma^{n}\right]_{+}=2 \eta^{k n}, \quad \eta_{k n}=\operatorname{diag}(\underbrace{1,-1, \ldots,-1}_{D+1}), k, n=0, \ldots, D .}
\end{aligned}
$$

Em termos dessas matrizes a equação (5.22) toma a forma

$$
\tilde{P}_{n} \Gamma^{n} \tilde{G}^{c}(x, y)=\delta^{D}(x-y), \quad \tilde{P}_{\mu}=i \partial_{\mu}-g A_{\mu}\left(x^{\mu}+\frac{i}{2} \theta^{\mu \nu} \partial_{\nu}\right), \quad \tilde{P}_{D}=-m .
$$

Agora de novo, semelhante ao (5.8), representamos $\tilde{G}^{c}(x, y)$ como o elemento de matriz do operador $\hat{G}^{c}$ (na representação de coordenadas $(5.10)$ ),

$$
\tilde{G}_{a b}^{c}(x, y)=<x\left|\hat{G}_{a b}^{c}\right| y>, \quad a, b=1,2, \ldots, 2^{d},
$$

onde, os índeces spinoriais $a, b$ são escritos aqui para serem melhor explicados e serão omitidos posteriormente. A equação (5.24) implica $\hat{S}^{c}=\left(\Pi_{n} \Gamma^{n}\right)^{-1}$, onde $\Pi_{\mu}$ são defenidos em (5.11), e $\Pi_{D}=-m$. Usando a generalização da representação do tempo próprio de Schwinger [74], escrevemos a função de Green (5.25) na forma

$$
\begin{aligned}
& \tilde{G}^{c}=\tilde{G}^{c}\left(x_{\text {out }}, x_{\text {in }}\right)=\int_{0}^{\infty} d \lambda \int\left\langle x_{\text {out }}\left|e^{-i \hat{\mathcal{H}}(\lambda, \chi)}\right| x_{\mathrm{in}}\right\rangle d \chi, \\
& \hat{\mathcal{H}}(\lambda, \chi)=\lambda\left(m^{2}-\Pi^{2}+\frac{i g}{2} F_{\mu \nu} \Gamma^{\mu} \Gamma^{\nu}\right)+\Pi_{n} \Gamma^{n} \chi .
\end{aligned}
$$

Semelhante ao [74], apresentamos o elemento de matriz que entra na expressão (5.26) por meio do integral de trajetória Hamiltoniano

$$
\begin{aligned}
& \tilde{G}^{c}=\exp \left(i \Gamma^{n} \frac{\partial_{l}}{\partial \varepsilon^{n}}\right) \int_{0}^{\infty} d \lambda_{0} \int d \chi_{0} \int_{\lambda_{0}} D \lambda \int_{\chi_{0}} D \chi \int_{x_{i n}}^{x_{\text {out }}} D x \int D p \int D \pi \int D \nu \\
& \times \int_{\psi(0)+\psi(1)=\varepsilon} \mathcal{D} \psi \exp \left\{i \int _ { 0 } ^ { 1 } \left[\lambda\left(\mathcal{P}^{2}-m^{2}+2 i g F_{\mu \nu}^{\star} \psi^{\mu} \psi^{\nu}\right)+2 i \mathcal{P}_{n} \psi^{n} \chi\right.\right. \\
& \left.\left.-i \psi_{n} \dot{\psi}^{n}+p_{\mu} \dot{x}^{\mu}+\pi \dot{\lambda}+\nu \dot{\chi}\right] d \tau+\psi_{n}(1) \psi^{n}(0)\right\}\left.\right|_{\varepsilon=0} .
\end{aligned}
$$

Aqui $\varepsilon^{n}$ são variaveis impares, que anticomutam com as matrizes $\Gamma$,

$$
\mathcal{P}_{\mu}=-p_{\mu}-g A_{\mu}\left(x^{\mu}-\frac{1}{2 \hbar} \theta^{\mu \nu} p_{\nu}\right), \mathcal{P}_{D}=-m, F_{\mu \nu}^{\star}=F_{\mu \nu}^{\star}\left(x^{\mu}-\frac{1}{2 \hbar} \theta^{\mu \nu} p_{\nu}\right),
$$


a função $F_{\mu \nu}^{\star}(q)$ é definida em (5.12), e a integração vai sobre as trajetórias pares $x(\tau), p(\tau), \lambda(\tau), \pi(\tau)$, e as trajetórias impares $\psi_{n}(\tau), \chi(\tau), \nu(\tau)$, parametrizadas por um parâmetro invariante $\tau \in[0,1]$ e obedecem as condições de fronteira $x(0)=x_{\text {in }}, x(1)=x_{\text {out }}$, $\lambda(0)=\lambda_{0}, \chi(0)=\chi_{0}$.

Fazendo a troca de variáveis (5.16) em (5.27), obtemos outra representação para $\tilde{G}^{c}$,

$$
\begin{aligned}
& \tilde{G}^{c}=\exp \left(i \Gamma^{n} \frac{\partial_{l}}{\partial \varepsilon^{n}}\right) \int_{0}^{\infty} d \lambda_{0} \int d \chi_{0} \int_{\lambda_{0}} D \lambda \int_{\chi_{0}} D \chi \int_{-\infty}^{\infty} D p \int_{x_{\text {in }}-\theta p / 2 \hbar}^{x_{\text {out }}-\theta p / 2 \hbar} D q \int D \pi \int D \nu \\
& \times\left.\int_{\psi(0)+\psi(1)=\varepsilon} \mathcal{D} \psi \exp \left\{i\left[S_{\text {spin-part }}^{\theta}+S_{\mathrm{GF}}\right]+\psi_{n}(1) \psi^{n}(0)\right\}\right|_{\varepsilon=0},
\end{aligned}
$$

com

$$
\begin{aligned}
& S_{\text {spin-part }}^{\theta}=\int_{0}^{1}\left[\lambda\left(\left(p_{\mu}+g A_{\mu}\right)^{2}-m^{2}+2 i g F_{\mu \nu}^{\star} \psi^{\mu} \psi^{\nu}\right)+2 i\left(p_{\mu}+g A_{\mu}(q)\right) \psi^{\mu} \chi\right. \\
& \left.-2 i m \psi^{D} \chi-i \psi_{n} \dot{\psi}^{n}+p_{\mu} \dot{q}^{\mu}-\frac{1}{2 \hbar} \dot{p}_{\mu} \theta^{\mu \nu} p_{\nu}\right] d \tau, \\
& S_{\mathrm{GF}}=\int_{0}^{1}(\pi \dot{\lambda}+\nu \dot{\chi}) d \tau .
\end{aligned}
$$

\subsection{Ação pseudoclássica da partícula spinorial no espaço-tempo não-comutativo}

Semelhante para o caso sem spin, o argumento do exponente na integral (5.28) pode ser considerado como a ação efetiva Hamiltoniana da partícula spinorial no espaço-tempo não-comutativo. Ele consiste de duas partes. A primeira $S_{\mathrm{GF}}$ com os derivados da $\lambda$ e $\chi$ pode ser tratada como o termo da fixação de gauge e corresponde, de fato, as condições de gauge $\dot{\lambda}=\dot{\chi}=0$. A parte restante $S_{\text {spin-part }}^{\theta}$ pode ser tratada como a ação invariante de gauge da partícula spinorial no

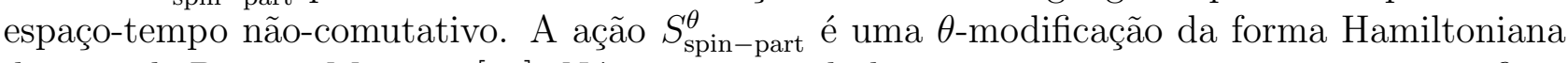
da ação de Berezin-Marinov [68]. Nós vamos estuda-la e construir a quantização para justificar esta interpretação.

Pode-se ver facilmente que $S_{\text {spin-part }}^{\theta}$ é invariante sobre a reparametrização. Aqui infelizmente não é fácil derivar a forma explícita das transformações da supersimetria. A presença da supersimetria será provada de jeito indireto. Mostraremos a existência de dois vínculos primários da primeira classe na formulação Hamiltoniana.

Vamos considerar $S_{\text {spin-part }}^{\theta}$ como a ação Lagrangiana com as coordenadas generalizadas $Q_{A}=\left(q^{\mu}, p_{\mu}\right), A=(\zeta, \mu), \zeta=1,2, Q_{1 \mu}=q^{\mu}, Q_{2 \mu}=p_{\mu} ; \chi, \psi$, e $\lambda$, e vamos fazer a Hamiltonização desta ação. Para este fim, introduzimos as momentas canônicas $P$ conjugadas 
às coordenadas generalizadas:

$$
\begin{aligned}
& P_{Q_{A}}=\frac{\partial L}{\partial \dot{Q}^{A}}=J_{A}(Q), J_{1 \mu}=p_{\mu}, \quad J_{2 \mu}=\frac{1}{2 \hbar} \theta^{\mu \nu} p_{\nu} \\
& P_{\lambda}=\frac{\partial L}{\partial \dot{\lambda}}=0, P_{\chi}=\frac{\partial_{r} L}{\partial \dot{\chi}}=0, P_{n}=\frac{\partial_{r} L}{\partial \dot{\psi}^{n}}=-i \psi_{n} .
\end{aligned}
$$

É fácil ver das equações (5.30) que existem os vínculos primários $\Phi^{(1)}=0$,

$$
\Phi_{l}^{(1)}=\left\{\begin{array}{l}
\Phi_{1 A}^{(1)}=P_{A}-J_{A}(Q) \\
\Phi_{2}^{(1)}=P_{\lambda}, \quad \Phi_{3}^{(1)}=P_{\chi} \\
\Phi_{4 n}^{(1)}=P_{n}+i \psi_{n} .
\end{array}\right.
$$

Os parênteses de Poisson dos vínculos primárioas são

$$
\begin{aligned}
& \left\{\Phi_{1 A}^{(1)}, \Phi_{1 B}^{(1)}\right\}=\Omega_{A B}=\left(\begin{array}{cc}
\mathbf{0} & \mathbb{I} \\
-\mathbb{I} & \theta / \hbar
\end{array}\right),\left\{\Phi_{4 n}^{(1)}, \Phi_{4 m}^{(1)}\right\}=2 i \eta_{n m} \\
& \left\{\Phi_{1 A}^{(1)}, \Phi_{4 n}^{(1)}\right\}=\left\{\Phi_{1 A}^{(1)}, \Phi_{2,3}^{(1)}\right\}=\left\{\Phi_{4 n}^{(1)}, \Phi_{2,3}^{(1)}\right\}=0
\end{aligned}
$$

onde $\theta=\theta^{\mu \nu}$, I é uma matriz de identidade $D \times D$, e $\mathbf{0}$ significa $D \times D$ matriz zero. Note que $\operatorname{det} \Omega_{A B}=1$, e

$$
\omega^{A B}=\Omega_{A B}^{-1}=\left(\begin{array}{cc}
\theta / \hbar & -\mathbb{I} \\
\mathbb{I} & \mathbf{0}
\end{array}\right) .
$$

Agora construímos o Hamiltoniano completo $H^{(1)}$, de acordo com o procedimento padrão [49]. Assim, obtemos:

$$
\begin{aligned}
& H^{(1)}=H+\Lambda_{l} \Phi_{l}^{(1)} \\
& H=-\lambda\left[\left(p_{\mu}+g A_{\mu}\right)^{2}-m^{2}+2 i g F_{\mu \nu}^{\star}(q) \psi^{\mu} \psi^{\nu}\right]+2 i \chi\left(\left(p_{\mu}+g A_{\mu}\right) \psi^{\mu}-m \psi^{D}\right) .
\end{aligned}
$$

onde $\Lambda_{l}$ são multiplicadores de Lagrange. As condições da consistência $\dot{\Phi}_{1 A, 4 n}^{(1)}=$ $\left\{\Phi_{1 A, 4 n}^{(1)}, H^{(1)}\right\}=0$ para os vínculos primários $\Phi_{1 A}^{(1)}$ e $\Phi_{4 n}^{(1)}$ admitem fixar os multiplicadores de Lagrange $\lambda^{1 A}$ e $\lambda^{4 n}$. As condições da consistência para os vínculos $\Phi_{2,3}^{(1)}$ implicam a existência dos vínculos secundários $\Phi_{1,2}^{(2)}=0$,

$$
\begin{aligned}
& \Phi_{1}^{(2)}=\left(p_{\mu}+g A_{\mu}\right) \psi^{\mu}-m \psi^{D}=0, \\
& \Phi_{2}^{(2)}=\left(p_{\mu}+g A_{\mu}\right)^{2}-m^{2}+2 i g F_{\mu \nu}^{\star} \psi^{\mu} \psi^{\nu}=0 .
\end{aligned}
$$

Assim, o Hamiltoniano $H$ é proporcional aos vínculos, como sempre no caso da teoria invariante da reparametrização,

$$
H=2 i \chi \Phi_{1}^{(2)}-\lambda \Phi_{2}^{(2)}
$$


Nenhum outro vínculo aparece do procedimento de Dirac, e os multiplicadores de Lagrange $\lambda^{2}$ e $\lambda^{3}$ permanecem indeterminados. Isto fica em uma correspondência perfeita com o fato que o número dos parâmetros das transformações de gauge é igual a dois.

Podemos passar do conjunto inicial dos vínculos $\left(\Phi^{(1)}, \Phi^{(2)}\right)$ para um conjunto equivalente $\left(\Phi^{(1)}, T\right)$, onde:

$$
T=\Phi^{(2)}+\frac{\partial \Phi^{(2)}}{\partial q^{A}} \omega^{A B} \Phi_{1 B}^{(1)}+\frac{i}{2}{\frac{\partial}{r} \Phi^{(2)}}_{\partial \psi^{n}}^{(1)} \Phi_{4 n}
$$

O novo conjunto dos vínculos pode ser dividido explicitamente por um conjunto dos vínculos da primeira clásse, que são $\left(\Phi_{2,3}^{(1)}, T\right)$ e um outro conjunto dos vínculos da segunda classe, que são $\left(\Phi_{1 A}^{(1)}, \Phi_{4 n}^{(1)}\right)$.

Agora podemos considerar a quantização dos operadores. Para isso fazemos fixação parcial de gauge, colocando $\Phi_{1,2}^{\mathrm{G}}=0$ para os vínculos primários da primeira classe $\Phi_{1,2}^{(1)}$,

$$
\Phi_{1}^{\mathrm{G}}=\chi=0, \quad \Phi_{2}^{\mathrm{G}}=\lambda=1 / m .
$$

Pode-se verificar que as condições da coerência das condições de gauge (5.36) levam à fixação dos multiplicadores de Lagrange $\lambda_{2}$ e $\lambda_{3}$. Assim, nesta etapa reduzimos nossa teoria Hamiltoniana para a teoria com vínculos de primeira classe $T$ e os de segunda classe $\varphi=\left(\Phi^{(1)}, \Phi^{\mathrm{G}}\right)$. Depois aplicamos o método da quantização de Dirac para os sistemas com vínculos da primeira classe [88], que sendo generalizado para o caso da presença dos vínculos de segunda classe pode ser formulado como: as relações de comutação entre os operadores são calculados de acordo com parênteses de Dirac em respeito aos somente vínculos de segunda classe; os vínculos de segunda classe como os operadores são egual a zero; os vínculos de primeira classe como os operadores não são zero, mas são considerados no senso da restrição em vetores do estado. Todas as equações dos operadores devem ser realizadas no espaço de Hilbert.

O sub-conjunto dos vínculos da segunda classe $\left(\Phi_{2,3}^{(1)}, \Phi^{\mathrm{G}}\right)$ tem a forma específica [49], assim que podem ser usado para a eliminar as variáveis $\lambda, P_{\lambda}, \chi, P_{\chi}$, da consideração, e depois para às variaveis restantes $q, p, \psi^{n}$, parênteses de Dirac em respeito aos vínculos $\varphi$ reduza para parênteses de Dirac em respeito somente aos vínculos $\Phi_{1 A}^{(1)}$ e $\Phi_{4 n}^{(1)}$ e pode ser facilmente calculada,

$$
\left\{Q^{A}, Q^{B}\right\}_{D\left(\Phi^{(1)}\right)}=\omega^{A B}, \quad\left\{\psi^{n}, \psi^{m}\right\}_{D\left(\Phi^{(1)}\right)}=\frac{i}{2} \eta^{n m}
$$

enquanto todos os outros parênteses de Dirac zeram. Assim, as relações de comutação para os operadores $\hat{q}^{\mu}, \hat{p}_{\mu}, \hat{\psi}^{n}$, que correspondem as variáveis $q^{\mu}, p_{\mu}, \psi^{n}$ são

$$
\begin{aligned}
& {\left[\hat{q}^{\mu}, \hat{p}_{\nu}\right]_{-}=i \hbar \omega^{\mu, D+\nu}=i \hbar \delta_{\nu}^{\mu},\left[\hat{q}^{\mu}, \hat{q}^{\nu}\right]=i \hbar \omega^{\mu \nu}=i \theta^{\mu \nu},\left[\hat{p}_{\mu}, \hat{p}_{\nu}\right]=0,} \\
& {\left[\hat{\psi}^{m}, \hat{\psi}^{n}\right]_{+}=i\left\{\psi^{m}, \psi^{n}\right\}_{D\left(\Phi^{(1)}\right)}=-\frac{1}{2} \eta^{m n} .}
\end{aligned}
$$


Além disso, existem as seguintes equações de operadores:

$$
\hat{\Phi}_{1 A}^{(1)}=\hat{P}_{A}-J_{A}(\hat{Q}), \hat{\Phi}_{4 n}^{(1)}=\hat{P}_{n}+i \hat{\psi}_{n}=0 .
$$

Levando isso em conta, pode-se construir uma realização das relações de comutação (5.37) no espaço de Hilbert cujos elementos $\Psi$ são colunas de $2^{d}$-componentes e dependem somente de $x$, assim que

$$
\hat{q}^{\mu}=\left(x^{\mu}+\frac{i}{2} \theta^{\mu \nu} \partial_{\nu}\right) \mathbf{I}, \quad \hat{p}_{\mu}=-i \partial_{\mu} \mathbf{I}, \quad \hat{\psi}^{n}=\frac{i}{2} \Gamma^{n},
$$

onde I é $2^{d} \times 2^{d}$ matriz de unidade e $\Gamma^{n}$ são matrizes gama (5.23). Os vínculos da primeira classe $\hat{T}$ como os operadores têm que aniquilar os vetores físicos; em virtude de (5.38) e (5.35) isso implica as equações:

$$
\hat{\Phi}_{1}^{(2)} \Psi=0, \quad \hat{\Phi}_{2}^{(2)} \Psi=0,
$$

onde $\hat{\Phi}_{1,2}^{(2)}$ são operadores que correspondem aos vínculos (5.33), (5.34). Tendo em vista as realizações das relações de comutação (5.37), pode-se ver facilmente que a primeira equação (5.40) tem a forma da equação de Dirac $\theta$-modificada,

$$
\left(\tilde{P}_{\mu} \tilde{\gamma}^{\mu}-m \gamma^{D+1}\right) \Psi=0 \Longleftrightarrow\left(P_{\mu} \gamma^{\mu}+m\right) \star \Psi=0
$$

Pois $\hat{\Phi}_{2}^{(2)}=\left(\hat{\Phi}_{1}^{(2)}\right)^{2}$, a segunda equação (5.40) é uma consequencia da primeira.

Assim temos construido a $\theta$-modificação da ação de Berezin-Marinov (5.29a) que após a quantização leva a teoria quântica baseada na equação de Dirac $\theta$-modificada.

Note que a não-comutatividade do espaço-tempo $\left[\hat{q}^{0}, \hat{q}^{i}\right]=i \theta^{0 i}$ pode ser obtida também da quantização canônica de uma ação Lagrangiana convencional da partícula relativística sem spin se impor a condição de gauge especial $\Phi_{g f}=x^{0}+\theta^{0 i} p_{i}-\tau=0$, veja [89].

\subsection{Integral de trajetória na mecânica quântica não- relativística no espaço não-comutativo}

Nesta seção construimos a representaçã via integral de trajetória para a função da propagação (o símbolo do operador da evolução) na mecânica quântica não-relativística no espaço nãocomutativo. Comparamos o nosso resultado com algumas construções anteriores e usamos ele para extrair as ações $\theta$-modificadas para este sistema.

Consideramos a mecânica quântica não-relativística $d$-dimensional com os básicos operadores canônicos das coordenadas $\hat{q}^{k}$ e das momentas $\hat{p}_{j}, k, j=1, \ldots, d$ que obedecem as seguintes relações de comutação

$$
\left[\hat{q}^{k}, \hat{q}^{j}\right]=i \theta^{k j},\left[\hat{q}^{k}, \hat{p}_{j}\right]=i \hbar \delta_{j}^{k},\left[\hat{p}_{k}, \hat{p}_{j}\right]=0 .
$$


A evolução do sistema com tempo é governada por um Hamiltoniano auto-adjunto $\hat{H}$. Acreditamos que para tal teoria quântica corresponde uma teoria clássica com ação $\theta$-modificada (que nós vamos procurar), tal que a quantização dela levaria MQNC descrita acima.

Na mecânica quântica convencional, a integral de trajetória aparece como a representação dos elementos de matriz (na representação das coordenadas) do operador da evolução $\hat{U}\left(t, t^{\prime}\right)$. Na MQNC também começamos com este operador. Ele obedece a equação de Schrödinger e se $\hat{H}$ independente do tempo (que nós consideramos para simplificar) tem a forma

$$
\hat{U}\left(t^{\prime}, t\right)=\exp \left\{-\frac{i}{\hbar} \hat{H}\left(t^{\prime}-t\right)\right\} .
$$

Já que os operadores de coordenadas $\hat{q}$ não comutam, eles não têm um comum conjunto completo dos autovetores. Por isso não existe a representação das coordenadas $q$ e não se fala sobre os elementos de matriz nesta representação. Consequentemente, não pode-se definir uma amplitude da probabilidade de uma transição entre os dois pontos no espaço de posição. No entanto, podemos considerar um outro tipo dos elementos de matriz do operador da evolução que são amplitudes de probabilidade (funções da evolução) e podem ser representados via integrais de trajetória. Abaixo, consideramos dois tipos de tais elementos de matriz,

$$
G_{p}=\left\langle p^{\text {out }}\left|\hat{U}\left(t_{\text {out }}, t_{\text {in }}\right)\right| p^{\text {in }}\right\rangle \text { and } G_{x}=\left\langle x_{\text {out }}\left|\hat{U}\left(t_{\text {out }}, t_{\text {in }}\right)\right| x_{\text {in }}\right\rangle .
$$

Em (5.44) $|p\rangle$ é um conjunto completo de autovetores dos operadores que comutam $\hat{p}$,

$$
\begin{gathered}
\hat{p}_{j}|p\rangle=p_{j}|p\rangle,<p\left|p^{\prime}>=\delta\left(p-p^{\prime}\right), \int\right| p><p \mid d p=I, d p=\prod_{i} d p_{i}, \\
<p\left|x>=\frac{1}{(2 \pi \hbar)^{d / 2}} \exp \left\{-\frac{i}{\hbar} p_{i} x^{i}\right\},<p\right| \hat{x}\left|p^{\prime}>=i \hbar \frac{\partial}{\partial p}<p\right| p^{\prime}>
\end{gathered}
$$

e $|x\rangle$ é um conjunto completo de autovetores dos operadores $\hat{x}^{k}$ que comutam mutualmente e são conjugados aos $\hat{p}$. Escolhemos estes operadores como:

$$
\begin{aligned}
& \hat{x}^{k}=\hat{q}^{k}+\frac{\theta^{k j} \hat{p}_{j}}{2 \hbar},\left[\hat{x}^{k}, \hat{x}^{j}\right]=0,\left[\hat{x}^{k}, \hat{p}_{j}\right]=i \hbar \delta_{j}^{k}, \\
& \hat{x}^{\mu}|x\rangle=x^{\mu}|x\rangle,\left\langle x\left|y>=\delta^{D}(x-y), \quad \int\right| x><x\right| d x=I, d x=\prod_{i} d x^{i} .
\end{aligned}
$$

Primeiramente, vamos construir a integral de trajetória para a função da evolução $G_{p}$. Para este fim, como sempre, dividimos o intervalo do tempo $T=t_{\text {out }}-t_{\text {in }}$ por $N$ partes eguais $\Delta t=T / N$ usando os pontos $t_{k}, k=1 \ldots N-1$, tais que $t_{k}=t_{i n}+k \Delta t$. Usando a propriedade de grupo do operador da evolução e a relação da completeness (veja (5.45)) para o conjunto $|p\rangle$, podemos escrever

$$
G_{p}=\lim _{N \rightarrow \infty} \int_{-\infty}^{\infty} d p^{(1)} \ldots d p^{(N-1)} \prod_{k=1}^{N}<p^{(k)}\left|\exp \left\{-\frac{i}{\hbar} \hat{H}\left(t_{k}-t_{k-1}\right)\right\}\right| p^{(k-1)}>
$$


onde $p^{(0)}=p^{(i n)}, p^{(N)}=p^{(\text {out })}$, e $p^{(k)}=\left(p_{i}^{(k)}\right)$. Tendo em vista o limite $N \rightarrow \infty$ ou $\Delta t \rightarrow 0$ e usando a relação de completeness (5.46) para autovetores $|x\rangle$, podemos calcular aproximadamente o elemento de matriz (5.47),

$$
<p^{(k)}\left|\exp \left\{-\frac{i}{\hbar} \hat{H} \Delta t\right\}\right| p^{(k-1)}>\approx \int d x_{(k)}<p^{(k)}\left|1-\frac{i}{\hbar} \hat{H} \Delta t\right| x_{(k)}><x_{(k)} \mid p^{(k-1)}>,
$$

com $x_{(k)}=\left(x_{(k)}^{i}\right)$ e $d x_{(k)}=\prod_{i} d x_{(k)}^{i}$. O resultado deste cálculo pode ser expresso em termos de um Hamiltoniano clássico $H$, no entanto, no caso geral, depende da escolha da regra da correspondência entre a função clássica $H$ e o operador quântico $\hat{H}$. Para nossos cálculos escolhemos o ordenamento simétrico de Weyl. Neste caso o elemento de matriz (5.48) toma a forma

$$
\int \frac{d x_{(k)}}{(2 \pi \hbar)^{d}} \exp \left\{\frac{i}{\hbar}\left[-x_{(k)}^{i} \frac{p_{i}^{(k)}-p_{i}^{(k-1)}}{\Delta t}-H\left(x_{(k)}-\frac{\theta p^{(k) \prime}}{2 \hbar}, p^{(k) \prime}\right)\right] \Delta t+O\left(\Delta t^{2}\right)\right\},
$$

onde $p^{(k) \prime}=\frac{p^{(k)}+p^{(k-1)}}{2}$, e $H\left(x-\frac{\theta p}{2 \hbar}, p\right)$ é o símbolo de Weyl do operador $\hat{H}$. Usando a formula acima e tomando o limite $N \rightarrow \infty$ no integral (5.47), obtemos para $G_{p}$ a seguinte representação via integral de trajetória:

$$
G_{p}=\int_{p^{(i n)}}^{p^{(o u t)}} D p \int D x \exp \left\{\frac{i}{\hbar} \int d t\left[-x_{j} \dot{p}^{j}-H\left(x-\frac{\theta p}{2 \hbar}, p\right)\right]\right\} .
$$

Da mesma forma, podemos construir a representação via integral de trajetória para a função da evolução $G_{x}$, que é

$$
G_{x}=\int D p \int_{x_{(\text {in })}}^{x_{(\text {out })}} D x \exp \left\{\frac{i}{\hbar} \int d t\left[p_{j} \dot{x}^{j}-H\left(x-\frac{\theta p}{2 \hbar}, p\right)\right]\right\} .
$$

Vamos passar para a integração sobre as trajetórias $q=x-\frac{\theta p}{2 \hbar}$ nas integrais de trajetória (5.49) e (5.50). Assim, temos

$$
\begin{aligned}
G_{x} & =\int D p \int_{x_{(\text {in })}-\theta p / 2 \hbar}^{x_{(\text {out })}-\theta p / 2 \hbar} D q \exp \left\{\frac{i}{\hbar} S_{\text {nonrel }}^{\theta}\right\}, \\
G_{p} & =\int_{p_{\text {in }}}^{p_{\text {out }}} D p \int D q \exp \left\{\frac{i}{\hbar} \tilde{S}_{\text {nonrel }}^{\theta}\right\},
\end{aligned}
$$

onde

$$
\begin{aligned}
& S_{\text {nonrel }}^{\theta}=\int d t\left[p_{j} \dot{q}^{j}-H(p, q)-\dot{p}_{j} \theta^{j i} p_{i} / 2 \hbar\right], \\
& \tilde{S}_{\text {nonrel }}^{\theta}=\int d t\left[-q_{j} \dot{p}^{j}-H(p, q)+p_{j} \theta^{j i} \dot{p}_{i} / 2 \hbar\right] .
\end{aligned}
$$


Note que as ações $S_{\text {nonrel }}^{\theta}$ e $\tilde{S}_{\text {nonrel }}^{\theta}$ se diferenciam por uma derivada total.

A integral de trajetória (5.51) é uma generalização do resultado obtido em [64] para o caso do sistema não-relativístico arbitrário e sem nenhuma restrição para a matriz $\theta$. Também notamos que as integrais de trajetórias no plano não-comutativo na representação dos estados coerentes foram estudados em [66] e [67]. Porém, elas têm uma forma específica que dificilmente pode ser comparada com nossos resultados. 


\section{Capítulo 6}

\section{Produto estrela através do ordenamento simétrico}

\subsection{Introdução}

Neste capítulo desenvolvemos uma abordagem para a construção do produto estrela na variedade de Poisson arbitrária. O método é baseado no ordenamento simétrico dos operadores $\hat{x}^{i}$, que representam coordenadas cartesianas deformadas em $\mathbb{R}^{N}$. Primeiro, requeremos que os $\hat{x}^{i}$ satisfaçam as relações de comutação gerais $\left[\hat{x}^{i}, \hat{x}^{j}\right]=2 \alpha \omega^{i j}(\hat{x})$, onde $\alpha$ é o parâmetro da deformação e $\omega^{i j}(x)=\omega_{0}^{i j}(x)+\sum \alpha^{n} \omega_{n}^{i j}(x)$, com $\omega_{0}^{i j}(x)$ sendo a estrutura de Poisson arbitrária. Depois, construímos a representação polidiferencial para $\hat{x}^{i}=x^{i}+\alpha \omega_{0}^{i j}(x) \partial_{j}+\ldots$ A maior vantagem da nossa abordagem é a existência de um procedimento iterativo por ordens do $\alpha$, tal que a ordem $(n-1)$ de $\hat{\omega}$ admite calcular a ordem $n$ de $\hat{x}$, que em torno define a ordem $n$ de $\hat{\omega}$. Além disso, operamos com objetos que são conhecidos da mecânica quântica. Alguns elementos da construção podem ser encontrados na literatura. Behr e Sykora [90] usaram o produto dos operadores simetricamente ordenados para construir o produto estrela em $\mathbb{R}^{N}$, mas sem o uso da representação polidiferencial dos operadores $\hat{x}$. As dificuldade técnicas não os admitiram em fazer o cálculo do produto estrela acima de segunda ordem da expansão.

Os cálculos do produto estrela de terceira ordem foram feitas em [75] usando as cohomologias de Hochschild e em [91] trocando as variáveis na fórmula de Moyal. Nosso resultado para esta ordem esta de perfeito acordo com [75]. As relações exatas entre o nosso produto estrela e o de [91] são menos claras, mas nós não vemos algumas contradições. 


\subsection{Ordenamento de Weyl e produto estrela}

Consideramos um conjunto dos operadores $\hat{x}^{j}, j=1, \ldots, N$ que não comutam e a função $f$ definida em $\mathbb{R}^{N}$ que pode ser apresentada na forma da série de Taylor,

$$
f(x)=\sum_{n=0}^{\infty} f_{i_{1} \ldots i_{n}}^{(n)} x^{i_{1}} \ldots x^{i_{n}}
$$

com $x^{i}$ sendo as coordenadas Cartesianas em $\mathbb{R}^{N}$. Associamos a esta função um operador simétricamente ordenado $\hat{f}(\hat{x})$ de acordo com a regra

$$
\hat{f}(\hat{x})=\sum_{n=0}^{\infty} f_{i_{1} \ldots i_{n}}^{(n)} \sum_{P_{n}} \frac{1}{n !} P_{n}\left(\hat{x}^{i_{1}} \ldots \hat{x}^{i_{n}}\right),
$$

onde $P_{n}$ são todas permutações dos $n$ elementos. Também usarémos outra notação,

$$
\hat{f} \equiv W(f) .
$$

Existe uma outra forma, mais conveniente, do ordenamento de Weyl. Se $\tilde{f}(p)$ é a transformação de Fourier da $f$,

$$
\tilde{f}(p)=\int d^{N} x f(x) e^{i p_{j} x^{j}}
$$

então

$$
\hat{f}(\hat{x})=\int \frac{d^{N} p}{(2 \pi)^{N}} \tilde{f}(p) e^{-i p_{m} \hat{x}^{m}} .
$$

Consideramos uma matriz anti-simétrica $\omega^{i j}(x)$ e vamos impor as relações de comutação dos operadores $\hat{x}$ :

$$
\left[\hat{x}^{i}, \hat{x}^{j}\right]=2 \alpha \hat{\omega}^{i j}(\hat{x}),
$$

onde $\alpha$ é um parâmetro da expansão. Na literatura física geralmente temos $\alpha=i \hbar / 2$. As relações de comutação (6.6) levam à condição de consistência

$$
\left[\hat{x}^{i}, \hat{\omega}^{j k}(\hat{x})\right]+\operatorname{cycl} \cdot(i j k)=0 .
$$

Vamos procurar a representação polidiferencial dos operadores $\hat{x}$ na forma da $\alpha$-expansão:

$$
\hat{x}^{i}=x^{i}+\sum_{n=1}^{\infty} \Gamma^{i(n)}(\alpha, x)(\alpha \partial)^{n},
$$

onde

$$
\Gamma^{i(n)}(\alpha, x)=\Gamma^{i i_{1} \ldots i_{n}}(\alpha, x)
$$


e cada um coeficiente $\Gamma$, é expandido em $\alpha$

$$
\Gamma^{i(n)}(\alpha, x)=\sum_{k=0}^{\infty} \alpha^{k} \Gamma_{k}^{i(n)}(x) .
$$

A matriz $\omega^{i j}(x)$ também é expandida na série de potências do $\alpha$. O termo de ordem $\alpha^{0}$ desta espansão $\omega_{0}^{i j}$ - é a estrutura de Poisson, ou seja, obedece a identidade de Jacobi

$$
\omega_{0}^{k l} \partial_{l} \omega_{0}^{i j}+\omega_{0}^{j l} \partial_{l} \omega_{0}^{k i}+\omega_{0}^{i l} \partial_{l} \omega_{0}^{k j}=0 .
$$

Já que os coeficientes $\Gamma^{i(n)}$ são contraídos em (2.6) com as derivadas parciais, somente a parte do $\Gamma^{i(n)}$ que é simétrica em últimos $n$ índices contribui na expressão (2.6). Por isso assumimos que $\Gamma^{i(n)}$ é simétrico em últimos $n$ índices.

Definimos o produto estrela como

$$
W(f \star g)=W(f) \cdot W(g) .
$$

Este produto é associativo por causa da associativedade do produto dos operadores. Para uma matriz $\omega^{i j}$ constante a fórmula (6.12) é bem conhecida da mecânica quântica [77]. Para as estruturas de Poisson lineares ela é a essência da construção de Gutt [92, 93]. Para matriz arbitrária $\omega^{i j}(x)$ esta definição do produto estrela foi usada em [90].

Também precisamos da seguinte propriedade: $f \star 1=f$, ou

$$
W(f) \cdot 1=f .
$$

As equações (6.12) e (6.13) levam a seguinte formula

$$
(f \star g)(x)=W(f) g(x)=\hat{f}(\hat{x}) g(x),
$$

onde o lado direito significa a ação do operador polidiferencial na função.

Existe uma simetria óbvia dessa construção que troca o sinal do $\alpha, \alpha \rightarrow-\alpha$, e reversa a ordem dos operadores. Esta simetria troca $f$ e $g$ em (6.12). A consequência desta simetria é seguinte, as ordens pares na $\alpha$-expansão do produto estrela são simétricas em relação a troca das $f$ e $g$, enquanto as ordens ímpares são anti-simétricas.

O produto estrela será construído usando um procedimento iterativo. Começamos com ordem $\alpha^{0}$ de $\omega$ que pode ser uma estrutura de Poisson arbitrária e resolvemos (6.6) na ordem $\alpha$ para obter a primeira ordem do coeficiente $\Gamma$ e dos operadores $\hat{x}^{j}$. Depois, usando estes operadores e a defenição do ordenamento de Weyl, construímos a primeira ordem do operador $\hat{\omega}^{i j}(\hat{x})$ em termos de $\omega_{0}$, substituímos ele na condição de consistência (6.7) e procuramos as correções para $\omega_{0}$. Estas correções podem significar que a matriz completa $\omega^{i j}(x)=\omega_{0}^{i j}(x)+$ $\sum \alpha^{n} \omega_{n}^{i j}(x)$ já não é mais o bivetor de Poisson. (Porém, até a ordem bastante alta em $\alpha$ a condição (6.7) é obedecida automaticamente e as correções para $\omega$ não aparecem). Depois temos que repetir o procedimento com essa nova $\omega$ na próxima ordem em $\alpha$. 


\subsection{Propriedades gerais da expansão}

\subsection{1 expansão de $\hat{x}$}

Consideramos as restrições para os coeficientes $\Gamma$ impostos pela condição (6.13). Esta condição é equivalente ao requerimento

$$
W\left(x^{i_{1}} \ldots x^{i_{n}}\right) \cdot 1=x^{i_{1}} \ldots x^{i_{n}}
$$

para todos monomiais das coordenadas e em todas ordens em $\alpha$. Note, que a parte direita de (6.15) não depende de $\alpha$. Consequêntemente, todas as contribuições na parte esquerda acima de ordem zero em $\alpha$ têm que zerar. Isto é equivalente a condição de que a simetrização completa de todos $\Gamma$ tem que ser igual a zero,

$$
\Gamma^{\left(i i_{1} \ldots i_{k}\right)}=0
$$

Ou a contraição de cada um dos $\Gamma^{(n)} \operatorname{com} n+1$ vetores $p_{i}$ que comutam tem que zerar,

$$
p_{i} p_{i_{1}} \ldots p_{i_{k}} \Gamma^{i i_{1} \ldots i_{k}}=0
$$

Chamaremos esta condição de traceless condição.

Agora vamos retornar às consequências das relações de comutação (6.6). Como vamos ver abaixo, a relação (6.6) admite expressar

$$
\Gamma_{k}^{\left[i i_{1}\right] i_{2} \ldots i_{p}} \equiv \Gamma_{k}^{i i_{1} i_{2} \ldots i_{p}}-\Gamma_{k}^{i_{1} i i_{2} \ldots i_{p}}
$$

com $k+p=n$ em termos de ordens mais baixas dos coeficientes $\Gamma$. É conveniente expandir a parte direita de (6.6) como

$$
\hat{\omega}^{i j}(\hat{x})=\hat{\omega}_{n}^{i j}+o\left(\alpha^{n}\right),
$$

onde $\hat{\omega}_{n}$ é de ordem $\alpha^{n}$. Também introduzimos as expansões correspondentes para $\hat{x}$ :

$$
\hat{x}^{j}=\hat{x}_{n}^{j}+o\left(\alpha^{n}\right), \quad \hat{x}_{n+1}^{j}=\hat{x}_{n}^{j}+\alpha^{n+1} \gamma_{n+1}^{j} .
$$

Vamos supor, que já foi calculada a expansão do operador $\hat{x}$ até a ordem $\alpha^{n}$, ou seja,

$$
\left[\hat{x}_{n}^{i}, \hat{x}_{n}^{j}\right]=2 \alpha \hat{\omega}_{n-1}+o\left(\alpha^{n}\right) .
$$

Depois temos que verificar a condição de consistência (6.7) em ordem $\alpha^{n}$ :

$$
\left[\hat{x}_{n}^{k}, \hat{\omega}_{n}^{i j}\right]+\left[\hat{x}_{n}^{j}, \hat{\omega}_{n}^{k i}\right]+\left[\hat{x}_{n}^{i}, \hat{\omega}_{n}^{j k}\right]=o\left(\alpha^{n}\right) .
$$

Para as ordens baixas esta equação é obedecida automaticamente para qualquer bivetor de Poisson $\omega^{i j}$. Nas ordens altas não é, e (6.22) tem que ser tratada como a equação para as correções para $\omega^{i j}$. A solução desta equação é um procedimento não trivial. Esta parte será considerada 
separadamente na seção 6.3.2. Por enquanto assumimos que as correções correspondentes são construídas e (6.22) é obedecida.

Para construir a próxima $(n+1)$ ordem na decomposição temos que resolver a seguinte equação

$$
\left[\hat{x}_{n+1}^{i}, \hat{x}_{n+1}^{j}\right]=2 \alpha \hat{\omega}_{n}^{i j}+o\left(\alpha^{n+1}\right)
$$

$\mathrm{Ou}$

$$
\left[\hat{x}_{n}^{i}+\alpha^{n+1} \gamma_{n+1}^{i}, \hat{x}_{n}^{j}+\alpha^{n+1} \gamma_{n+1}^{j}\right]=2 \alpha \hat{\omega}_{n}^{i j}+o\left(\alpha^{n+1}\right)
$$

que implica

$$
\left[\hat{x}_{n}^{i}, \hat{x}_{n}^{j}\right]+\alpha^{n+1} \gamma_{n+1}^{[i j]}=2 \alpha \hat{\omega}_{n}^{i j}+o\left(\alpha^{n+1}\right),
$$

onde

$$
\gamma_{n+1}^{[i j]} \equiv \sum_{p+k=n+1} p \Gamma_{k}^{[i j] i_{2} \ldots i_{p}} \partial_{i_{2}} \ldots \partial_{i_{p}}
$$

Por isso, a parte anti-simétrica do $\Gamma_{n+1}$ é determinada da equação

$$
\alpha^{n+1} \gamma_{n+1}^{[i j]}=G_{n+1}^{i j}+o\left(\alpha^{n+1}\right),
$$

com

$$
G_{n+1}^{i j}=2 \alpha \hat{\omega}_{n}^{i j}-\left[\hat{x}_{n}^{i}, \hat{x}_{n}^{j}\right]+o\left(\alpha^{n+1}\right) .
$$

Usando esta equação podemos definir $G_{n+1}^{i j}$ até a ordem $o\left(\alpha^{n+1}\right)$. Não incluímos nenhum termo de ordem mais álta no $G_{n+1}^{i j}$, assim que $G_{n+1}^{i j} \sim \alpha^{n+1}$. Os operadores $G_{n+1}^{i j}$ podem ser expandidos como

$$
G_{n+1}^{i j}=\sum_{p} G_{n+1}^{i j i_{2} \ldots i_{p}} \partial_{i_{2}} \ldots \partial_{i_{p}} .
$$

Os coeficientes nesta expansão são anti-simétricos em $i, j$ e simétricos em $i_{2}, \ldots, i_{p}$ pela construção. Além disso eles têm a seguinte propriedade.

Lemma 1 Os coeficientes $G_{n+1}^{i j i_{2} \ldots i_{p}}$ obedecem a condição cíclica

$$
G_{n+1}^{i j i_{2} \ldots i_{p}}+\operatorname{cycl} .\left(i j i_{2}\right)=0 .
$$

Proof. De (6.22) temos

$$
\left[\hat{x}_{n}^{k}, 2 \alpha \hat{\omega}_{n}^{i j}\right]+\operatorname{cycl} .(k i j)=o\left(\alpha^{n+1}\right),
$$

ou usando (6.27),

$$
\left[\hat{x}_{n}^{k}, G_{n+1}^{i j}+\left[\hat{x}_{n}^{i}, \hat{x}_{n}^{j}\right]\right]+\operatorname{cycl} .(k i j)=o\left(\alpha^{n+1}\right) .
$$

A equação

$$
\left[\hat{x}_{n}^{k},\left[\hat{x}_{n}^{i}, \hat{x}_{n}^{j}\right]\right]+\operatorname{cycl} .(k i j)=0
$$


permanece válida em todas ordens de $\alpha$, incluindo a ordem $\alpha^{n+1}$. Portanto, de (6.31) obtemos

$$
\left[x_{n}^{k}, G_{n+1}^{i j}\right]+c y c l=0 .
$$

Depois substituímos a expressão (6.28) em (6.33), calculamos o comutador, e usamos a simetria de $G_{n+1}$ em últimos $p-1$ índexes para completar a prova.

Por causa da simetria de $G_{n+1}^{i j i_{2} \ldots i_{p}}$ em últimos $p-1$ índices, a condição cíclica permanece válida para permutações de $\left(i, j, i_{k}\right)$ para qualquer $k=2, \ldots, p$.

Agora podemos construir $\Gamma_{p}^{i j \ldots k}$ bem como o operador $\hat{x}$ em ordem $\alpha^{n+1}$ simetrizando os coeficientes $G_{n+1}^{i j i_{1} \ldots i_{k}}$. As equações que têm que ser obedecidas por $\Gamma$ para resolver as relaçoes de comutação (6.23) têm a forma

$$
G_{n+1}^{j i_{1} \ldots i_{p}}=\alpha^{n+1} p \Gamma_{k}^{\left[j i_{1}\right] \ldots i_{p}}, \quad k+p=n+1 .
$$

A solução destas equações é dada pela seguinte Lema.

Lemma 2 Os tensores

$$
\Gamma_{k}^{j i_{1} \ldots i_{p}}=\frac{\alpha^{-(n+1)}}{p(p+1)}\left(G_{n+1}^{j i_{1} i_{2} \ldots i_{p}}+G_{n+1}^{j i_{2} i_{1} i_{3} \ldots i_{p}}+\cdots+G_{n+1}^{j i_{p} i_{1} i_{2} \ldots i_{p-1}}\right)
$$

são simétricos em últimos $p$ indeces, satisfaçam a equação (6.34) e tracelessness condição (6.16).

Proof. A simétria segue da construção e traceless condição é a consequência da antisimetria dos $G_{n+1}^{j i_{1} i_{2} \ldots i_{p}}$ em primeiros dois índices. Para provar a afirmação restante, consideramos as combinações anti-simetrizadas em $j$ e $i_{1}$ dos tensores que entram na parte direita de (6.34):

$$
G_{n+1}^{j i_{1} i_{2} \ldots i_{p}}-G_{n+1}^{i_{1} j i_{2} \ldots i_{p}}=2 G_{n+1}^{j i_{1} i_{2} \ldots i_{p}}
$$

por causa da anti-simetria do $G$ em primeiros dois índeces. Temos

$$
G_{n+1}^{j i_{2} i_{1} \ldots i_{p}}-G_{n+1}^{i_{1} i_{2} j \ldots i_{p}}=G_{n+1}^{j i_{1} i_{2} \ldots i_{p}}
$$

por causa da condição cíclica em $j, i_{1}, i_{2}$. Se tratar as restantes $p-2$ combinações do mesmo jeito, a afirmação segue imediatamente.

Esta Lema implica que os tensores (6.35) são coeficientes da expansão do operador $\hat{x}^{j}$ que nós procuramos. Para a conveniência definimos $\star_{k}$ que é uma parte do produto estrela na ordem $\alpha^{k}$.

$$
f \star g=\sum_{k=0}^{n} f \star_{k} g+O\left(\alpha^{n+1}\right) .
$$


Para calcular o produto estrela e $\hat{\omega}(\hat{x})$ até a ordem desejada de $\alpha$ precisamos da ferramenta efetiva para calcular a $\alpha$-expansão de $\hat{f}(\hat{x})$ para qualquer $f$ e qualquer expansão de $\hat{x}$. Para este fim vamos usar a representação integral (6.5) e a fórmula de Duhamel

$$
e^{A+B}=e^{A}+\int_{0}^{1} e^{(A+B) s} B e^{(1-s) A} d s .
$$

Aqui $A+B=-i p_{i} \hat{x}^{i}, A=-i p_{i} x^{i}$ e $B=-i p_{i}\left(\hat{x}^{i}-x^{i}\right)$. Já que, $B$ é de ordem $\alpha^{1}$, e $A$ é de ordem $\alpha^{0}$, mas por causa da propriedade (6.17), cada comutador é no mínimo uma ordem

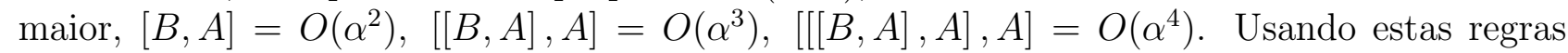
achamos,

$$
\begin{aligned}
e^{A+B} & =e^{A}\left(1+B+\frac{1}{2}[B, A]+\frac{1}{2} B^{2}\right. \\
& +\frac{1}{6}[[B, A], A]+\frac{1}{3}[B, A] B+\frac{1}{6} B[B, A]+\frac{1}{6} B^{3} \\
& +\frac{1}{24}[[[B, A], A], A]+\frac{1}{8}[[B, A], A] B+\frac{1}{8}[B, A]^{2} \\
& +\frac{1}{24} B[[B, A], A]+\frac{1}{8}[B, A] B^{2}+\frac{1}{12} B[B, A] B \\
& \left.+\frac{1}{24} B^{2}[B, A]+\frac{1}{24} B^{4}\right)+O\left(\alpha^{5}\right) .
\end{aligned}
$$

A estratégia geral do cálculo da $\alpha$-expansão do produto estrela é seguinte: começando com a ordem $n$ do operador $\hat{x}^{j}$ calculamos o operador $\hat{\omega}^{i j}\left(\hat{x}_{n}\right)$ e os operadores $G_{n+1}^{i j}$ de (6.27). Os operadores da próxima ordem são construídos usando Lema 2. Depois temos que verificar a condição de consistência nesta ordem e, se é necessário, calcular as correções para $\omega$. Tirando as correções para $\omega$, o procedimento proposto é automático e pode ser adaptado até para o cálculo do computador.

\subsubsection{Correções para $\omega$}

Aqui apresentamos descrição curta do que acontece caso as correções para $\omega$ são necessárias. A discussão mais detalhada pode ser encintrada em ?? abaixo, onde trabalhamos com o caso particular do produto estrela de terceira ordem. Vamos supor que nós completamos a nossa construção até a ordem $n$, ou seja, temos operadores $\hat{x}_{n}^{j}$ obedecendo as relações (6.21). Depois pegamos a função $\omega_{n-1}$ e construímos o operador correspondente $\hat{\omega}^{i j}\left(\hat{x}_{n}\right)$ e o produto estrela $\tilde{\star}_{n}$, onde til significa que ainda precisamos verificar a condição de consistência (6.22) na ordem $n$. Vamos assumir para simplicidade que em ordens mais baixas nenhuma correção para $\omega$ apareceu, ou seja, que em ordens mais baixas a condição de consistência foi obedecida para $\omega^{i j}=\omega_{0}^{i j}$. Vamos denotar $\tilde{\star} \equiv \star_{1}+\cdots+\star_{n-1}+\tilde{\star}_{n}$ e calcular

$$
x^{i} \tilde{\star} \omega^{j k}-\omega^{j k} \tilde{\star} x^{i}+\operatorname{cycl}(i j k) \text {. }
$$


Se a expressão (6.39) é $o\left(\alpha^{n}\right)$ para $\omega=\omega_{0}$, nenhuma correção é necessária nesta ordem também. Se a expressão (6.39) contem alguns termos de ordem $\alpha^{n}$ (os termos das ordens mais baixas zeram por causa das condições de consistência nas ordens mais baixas), temos que corregir $\omega$ e, consequentemente, o produto estrela. É fácil ver que nenhuma correção para $\omega$ de ordem $\alpha^{n}$ vai aparecer. Temos que corregir $\omega$ em ordem $\alpha^{n-1}$, assim que $\omega=\omega_{0}+\alpha^{n-1} \omega_{n-1}$. Isto parece muito perigoso para a abordagem completa já que vamos corregir a ordem que já foi construída. No entanto, isto não é assim. Consideramos com mais cuidado o que está acontecendo. O único efeito que a correção para $\omega$ de ordem $\alpha^{n-1}$ tem nos operadores $\hat{x}_{n}$ é que agora a parte $\Gamma_{n-1}^{i j}$ não é igual a zero,

$$
\Gamma_{n-1}^{i j}=\omega_{n-1}^{i j} .
$$

O produto estrela se modifica em ordem $n$ somente como, $\tilde{\star}_{n} \rightarrow \star_{n}$,

$$
f \star_{n} g=f \tilde{\star}_{n} g+\alpha^{n} \omega_{n-1}^{i j} \partial_{i} f \partial_{j} g .
$$

Esta fórmula está relacionada com a bem conhecida ambiguidade no produto estrela (veja, por exemplo, [75]). Se o produto estrela $\tilde{\star}$ é associativo até a ordem $\alpha^{n+1}$, então o produto estrela * também é associativo até a ordem $\alpha^{n+1}$ independentemente da escolha do $\omega_{n-1}^{i j}$. Portanto, os dois produtos são os legítimos produtos estrela. A única diferença é que $\approx$ não pode ser extendido até a próxima ordem com uso do nosso método, enquanto * pode. Se precisamos o produto estrela somente até a ordem $n$, podemos ignorar todas as correções que vêm da condição de consistência de ordem $n$ e ordens mais altas.

\subsection{Calculação do produto estrela}

\subsubsection{Produto estrela de primeira ordem}

Vamos calcular a ordem zero e primeira ordem na $\alpha$-expansão do produto estrela. Na ordem zero a condição (6.6) tem a forma $\left[\hat{x}^{i}, \hat{x}^{j}\right]=0$, assim que nós temos uma álgebra comutativa não-deformada, $\hat{x}^{j}=x^{j}$, e como foi esperado,

$$
f \star_{0} g=f \cdot g .
$$

Até a primeira ordem em $\alpha$ a expansão (6.8) tem a forma

$$
\hat{x}^{i}=x^{i}+\alpha \Gamma_{0}^{i j}(x) \partial_{j}+O\left(\alpha^{2}\right) .
$$

Substituindo esta expressão em (6.6) e deixando os termos apenas lineares em $\alpha$, obtemos

$$
\Gamma_{0}^{[i j]}=\Gamma_{0}^{i j}(x)-\Gamma_{0}^{j i}(x)=2 \omega^{i j}(x),
$$

que implica

$$
\Gamma_{0}^{i j}(x)=\omega^{i j}(x)+S_{0}^{i j}(x) .
$$


Note que até esta ordem em $\alpha$ o ordinamento de Weyl é trivial, $\hat{\omega}(\hat{x})=\omega(x)$. A parte simétrica $S_{0}^{i j}$ é eliminada pela traceless condição (6.16), $S_{0}^{i j}=0$, e

$$
\Gamma_{0}^{i j}(x)=\omega^{i j}(x) .
$$

Da fórmula de Duhamel (6.37) temos

$$
\begin{aligned}
e^{-i p_{m} \hat{x}^{m}} & =e^{A}+e^{A} B+o\left(\alpha^{1}\right) \\
& =e^{-i p_{m} x^{m}}-i \alpha e^{-i p_{m} x^{m}} p_{j} \omega^{j k}(x) \partial_{k}+O\left(\alpha^{2}\right) .
\end{aligned}
$$

Usando a equação (6.14) obtemos

$$
f \star_{1} g=\alpha \omega^{i j} \partial_{i} f \partial_{j} g
$$

e a expansão

$$
\hat{\omega}^{i j}(\hat{x})=\omega^{i j}(x)+\alpha \partial_{l} \omega^{i j}(x) \omega^{l k}(x) \partial_{k}+O\left(\alpha^{2}\right),
$$

que será usada abaixo para o cálculo de próxima ordem do produto estrela.

A condição de consistência (6.7) nesta ordem

$$
\left(x^{k} \star_{1} \omega^{i j}-\omega^{i j} \star_{1} x^{k}\right)+\operatorname{cycl} .(k i l)=0
$$

leva a identidade de Jacobi (6.11), ou seja, $\omega=\omega_{0}$ é o bivetor de Poisson. Vamos deixar de escrever o subscripto 0 nas notações abaixo.

\subsubsection{Produto estrela de segunda ordem}

Como pode-se ver da equação (6.49), o operador $\hat{\omega}^{i j}$ de ordem $\alpha^{1}$ é um operador diferencial de primeira ordem e não tem a parte sem derivadas. Consequentemente, $\gamma_{2}^{i}$ não tem a parte com derivadas de primeira ordem, e por isso, podemos escrever

$$
\hat{x}_{2}^{i}=x^{i}+\alpha \omega^{i j}(x) \partial_{j}+\alpha^{2} \Gamma_{0}^{i j k}(x) \partial_{j} \partial_{k} .
$$

As relaçoes de comutação (6.21) com $n=1$ levam

$$
2 \alpha^{2} \Gamma_{0}^{[i l] k}=G_{2}^{i l k}=2 \alpha^{2}\left(\omega^{j k} \partial_{j} \omega^{i l}+\frac{1}{2} \omega^{l j} \partial_{j} \omega^{i k}-\frac{1}{2} \omega^{i j} \partial_{j} \omega^{l k}\right)
$$

A condição cíclica

$$
G_{2}^{i l k}+G_{2}^{k i l}+G_{2}^{l k i}=0
$$

pode ser fácilmente verificada. Ela é equivalente a identidade de Jacobi para $\omega^{i j}$. Com ajuda desta identidade podemos reescrever (6.52) como

$$
G_{2}^{i l k}=\alpha^{2} \omega^{j k} \partial_{j} \omega^{i l}
$$


De acordo com a prescrição geral do Lema 2,

$$
\alpha^{2} \Gamma_{0}^{i l k}=\frac{1}{6}\left(G_{2}^{i l k}+G_{2}^{i k l}\right)
$$

$\mathrm{Ou}$,

$$
\Gamma_{0}^{i l k}=\frac{1}{6} \omega^{j k} \partial_{j} \omega^{i l}+\frac{1}{6} \omega^{j l} \partial_{j} \omega^{i k}
$$

Obviamente, $\Gamma_{0}^{i l k}$ obedece traceless condição (6.16). Eq. (6.52) pode ser verificada diretamente usando a identidade de Jacobi.

Agora podemos calcular o produto estrela de segunda ordem usando a formula (6.14). Primeiro, calculamos

$$
[B, A]=-\frac{\alpha^{2}}{3} p_{i} p_{k} \omega^{j i} \partial_{j} \omega^{k l} \partial_{l}+O\left(\alpha^{3}\right)
$$

A decomposição (6.38) leva

$$
\begin{aligned}
& e^{-i p_{i} \hat{x}^{i}}=e^{-i p_{m} x^{m}}-i \alpha e^{-i p_{m} x^{m}} p_{i} \omega^{i j}(x) \partial_{j} \\
& -\frac{\alpha^{2}}{2} e^{-i p_{m} x^{m}} p_{i} p_{k} \omega^{i j} \omega^{k l} \partial_{j} \partial_{l}-\frac{\alpha^{2}}{3} e^{-i p_{m} x^{m}} p_{i} p_{k} \omega^{i j} \partial_{j} \omega^{k l} \partial_{l} \\
& +\frac{i \alpha^{2}}{6} e^{-i p_{m} x^{m}} p_{k}\left(\omega^{j k} \partial_{j} \omega^{i l}+\omega^{j l} \partial_{j} \omega^{i k}\right) \partial_{i} \partial_{l}+O\left(\alpha^{3}\right) .
\end{aligned}
$$

Da eq. (6.5), temos

$$
\begin{aligned}
f(\hat{x}) & =f(x)+\alpha \omega^{i j} \partial_{i} f \partial_{j} \\
& +\frac{\alpha^{2}}{2} \omega^{i j} \omega^{k l} \partial_{i} \partial_{k} f \partial_{j} \partial_{l}+\frac{\alpha^{2}}{3} \omega^{i j} \partial_{j} \omega^{k l} \partial_{i} \partial_{k} f \partial_{l} \\
& -\frac{\alpha^{2}}{3}\left(\omega^{j k} \partial_{j} \omega^{i l}+\omega^{j l} \partial_{j} \omega^{i k}\right) \partial_{k} f \partial_{i} \partial_{l}+O\left(\alpha^{3}\right) .
\end{aligned}
$$

E depois, de (6.14) obtemos

$$
\begin{aligned}
& (f \star g)(x)=\hat{f}(\hat{x}) g(x)=f g+\alpha \partial_{i} f \omega^{i j} \partial_{j} g \\
& +\frac{\alpha^{2}}{2} \omega^{i j} \omega^{k l} \partial_{i} \partial_{k} f \partial_{j} \partial_{l} g+\frac{\alpha^{2}}{3} \omega^{i j} \partial_{j} \omega^{k l}\left(\partial_{i} \partial_{k} f \partial_{l} g-\partial_{k} f \partial_{i} \partial_{l} g\right)+O\left(\alpha^{3}\right) .
\end{aligned}
$$

Esta expressão coincide com a formula de Kontsevich [70]. A mesma expressão foi obtida da abordagem de Behr e Sykora [90]. Já que o produto (6.58) coincide com os bem conhecidos, não precisamos verificar (6.7) nesta ordem. A condição de consistência também segue da afirmação mais forte

$$
f \star_{2} g-g \star_{2} f=0,
$$

que é a consequência da simetria descrita na seção 6.2 e pode ser facilmente verificada usando (6.58). As correções para $\omega$ não aparecem, que significa que até esta ordem $\omega^{i j}=\omega_{0}^{i j}$ é uma estrutura de Poisson. 
Como a preparação para os cálculo da próxima ordem nós escrevemos a decomposição para $\hat{\omega}:$

$$
\begin{aligned}
\hat{\omega}^{i j}(\hat{x}) & =\omega^{i j}+\alpha \omega^{k l} \partial_{k} \omega^{i j} \partial_{l} \\
+ & \frac{\alpha^{2}}{2} \omega^{n k} \omega^{m l} \partial_{n} \partial_{m} \omega^{i j} \partial_{k} \partial_{l}+\frac{\alpha^{2}}{3} \omega^{n k} \partial_{k} \omega^{m l} \partial_{n} \partial_{m} \omega^{i j} \partial_{l} \\
- & \frac{\alpha^{2}}{3}\left(\omega^{n k} \partial_{n} \omega^{l m}+\omega^{n l} \partial_{n} \omega^{k m}\right) \partial_{m} \omega^{i j} \partial_{k} \partial_{l}+O\left(\alpha^{3}\right) .
\end{aligned}
$$

\subsubsection{Produto estrela de terceira ordem}

O operador $\hat{\omega}$ em ordem $\alpha^{2}$, eq. (6.60), bem como nas ordens anteriores, não contem a parte sem derivadas. Consequentemente, podemos escrever

$$
\begin{aligned}
& \hat{x}_{2}^{i}+\gamma_{3}^{i}=\hat{x}_{3}^{i}=x^{i}+\alpha \Gamma_{0}^{i j}(x) \partial_{j}+\alpha^{2} \Gamma_{0}^{i j k}(x) \partial_{j} \partial_{k}+ \\
& \alpha^{3}\left(\Gamma_{1}^{i j k}(x) \partial_{j} \partial_{k}+\Gamma_{0}^{i j k l}(x) \partial_{j} \partial_{k} \partial_{l}\right)+O\left(\alpha^{4}\right) .
\end{aligned}
$$

O coeficiente $\Gamma_{0}^{i j k}$ é sabido, enquanto $\Gamma_{1}^{i j k}$ e $\Gamma_{0}^{i j k l}$ têm que ser determinados. Comparando (6.60) com o comutador dos operadores de (6.61) obtemos (cf. (6.27))

$$
G_{3}^{i j k}=\frac{\alpha^{3}}{3} \omega^{n l}\left(2 \partial_{l} \omega^{m k} \partial_{n} \partial_{m} \omega^{i j}+\partial_{l} \omega^{m j} \partial_{n} \partial_{m} \omega^{i k}+\partial_{l} \omega^{m i} \partial_{n} \partial_{m} \omega^{k j}\right)
$$

e

$$
\begin{aligned}
G_{3}^{i j k l} & =\alpha^{3}\left(\omega^{n k} \omega^{m l} \partial_{n} \partial_{m} \omega^{i j}+\frac{1}{3} \omega^{k n} \partial_{n} \omega^{l m} \partial_{m} \omega^{i j}\right. \\
& +\frac{1}{3} \omega^{l n} \partial_{n} \omega^{k m} \partial_{m} \omega^{i j}+\omega^{j m} \partial_{m} \Gamma_{0}^{i k l}-\omega^{i m} \partial_{m} \Gamma_{0}^{j k l} \\
& \left.+\Gamma_{0}^{j k m} \partial_{m} \omega^{i l}+\Gamma_{0}^{j l m} \partial_{m} \omega^{i k}-\Gamma_{0}^{i k m} \partial_{m} \omega^{j l}-\Gamma_{0}^{i l m} \partial_{m} \omega^{j k}\right) .
\end{aligned}
$$

As identidades cíclicas (Lema 1) podem ser verificadas diretamente como as consequências da identidade de Jacobi. De acordo com Lema 2, temos

$$
\Gamma_{1}^{i j k}=\frac{1}{6} \omega^{n l} \partial_{l} \omega^{m k} \partial_{n} \partial_{m} \omega^{i j}+\frac{1}{6} \omega^{n l} \partial_{l} \omega^{m j} \partial_{n} \partial_{m} \omega^{i k} .
$$

e

$$
\alpha^{3} \Gamma_{0}^{i j k l}=\frac{1}{12}\left(G_{3}^{i j k l}+G_{3}^{i k j l}+G_{3}^{i l k j}\right) .
$$


Com ajuda dos comutadores (6.85), a fórmula de Duhamel (6.38), e as expressões (6.14), (6.62) - (6.65) calculamos o produto estrela de terceira ordem:

$$
\begin{aligned}
f \tilde{\star}_{3} g & =\alpha^{3}\left[\frac{1}{3} \omega^{n l} \partial_{l} \omega^{m k} \partial_{n} \partial_{m} \omega^{i j}\left(\partial_{i} f \partial_{j} \partial_{k} g-\partial_{i} g \partial_{j} \partial_{k} f\right)\right. \\
& +\frac{1}{6} \omega^{n k} \partial_{n} \omega^{j m} \partial_{m} \omega^{i l}\left(\partial_{i} \partial_{j} f \partial_{k} \partial_{l} g-\partial_{i} \partial_{j} g \partial_{k} \partial_{l} f\right) \\
& +\frac{1}{3} \omega^{l n} \partial_{l} \omega^{j m} \omega^{i k}\left(\partial_{i} \partial_{j} f \partial_{k} \partial_{n} \partial_{m} g-\partial_{i} \partial_{j} g \partial_{k} \partial_{n} \partial_{m} f\right) \\
& +\frac{1}{6} \omega^{j l} \omega^{i m} \omega^{k n} \partial_{i} \partial_{j} \partial_{k} f \partial_{l} \partial_{n} \partial_{m} g \\
& \left.+\frac{1}{6} \omega^{n k} \omega^{m l} \partial_{n} \partial_{m} \omega^{i j}\left(\partial_{i} f \partial_{j} \partial_{k} \partial_{l} g-\partial_{i} g \partial_{j} \partial_{k} \partial_{l} f\right)\right]
\end{aligned}
$$

Colocamos til em cima do produto estrela (6.66) para mostrar que ele ainda não inclui as correções para $\omega$ requeridas pela condição de consistência (6.22) em terceira ordem. Porém, (6.66) é completamente legítimo produto estrela. Ele somente não pode ser extendido até a quarta ordem no nosso procedimento. Eq. (6.66) fica de acordo com [75] e obedece os requerementos da associativedade [91].

\subsubsection{Correções para o produto estrela de terceira ordem}

Vamos estudar a condição da consistência em terceira ordem para o produto estrela da seção anterior. Temos que calcular (6.39) para $n=3$ e verificar se os termos de ordem $\alpha^{3}$ zeram. Isto implica na condição

$$
\begin{aligned}
& x^{a \tilde{\star}_{3} \omega^{b c}-\omega^{b c} \tilde{\star}_{3} x^{a}}+\operatorname{cycl}(a b c) \\
& =\alpha^{3}\left(\frac{2}{3} \omega^{n l} \partial_{l} \omega^{m k} \partial_{n} \partial_{m} \omega^{a j} \partial_{j} \partial_{k} \omega^{b c}+\frac{1}{3} \omega^{n k} \omega^{m l} \partial_{n} \partial_{m} \omega^{a j} \partial_{j} \partial_{k} \partial_{l} \omega^{b c}\right) \\
& +\operatorname{cycl}(a b c)=0 .
\end{aligned}
$$

A condição (6.67) não é obedecida por qualquer $\omega^{b c}=\omega_{0}^{b c}$, ou seja, ela não segue da identidade de Jacobi [75]. Portanto precisamos construir as correções para $\omega_{0}$ e de acordo com seção 6.3.2, elas têm que ser de ordem $\alpha^{2}$ :

$$
\omega^{b c}(x)=\omega_{0}^{b c}(x)+\alpha^{2} \omega_{2}^{b c}(x)+\ldots
$$

Isto leva ao fato que $\Gamma_{2}^{i j}$ não é zero, de acordo com a equação

$$
\Gamma_{2}^{i j}-\Gamma_{2}^{j i}=2 \omega_{2}^{i j},
$$

que junto com traceless condição leva

$$
\Gamma_{2}^{i j}=\omega_{2}^{i j}
$$


O produto estrela de terceira ordem'se modifica $\tilde{\star}_{3} \rightarrow \star_{3}$, como

$$
f \star_{3} g=f \tilde{\star}_{3} g+\alpha^{3} \partial_{i} f \omega_{2}^{i j} \partial_{j} g .
$$

$\omega_{2}^{i j}$ tem que ser determinada da condição de consistência que tem a forma

$$
\begin{aligned}
& x^{a} \star_{3} \omega_{0}^{b c}-\omega_{0}^{b c} \star_{3} x^{a}+\alpha^{2} x^{a} \star_{1} \omega_{2}^{b c}-\alpha^{2} \omega_{2}^{b c} \star_{1} x^{a}+\operatorname{cycl} .(a b c) \\
& =\alpha^{3}\left[2 \omega_{0}^{a d} \partial_{d} \omega_{2}^{b c}+2 \omega_{2}^{a d} \partial_{d} \omega_{0}^{b c}+\frac{2}{3} \omega_{0}^{n l} \partial_{l} \omega_{0}^{m k} \partial_{n} \partial_{m} \omega_{0}^{a j} \partial_{j} \partial_{k} \omega_{0}^{b c}\right. \\
& \left.+\frac{1}{3} \omega_{0}^{n k} \omega_{0}^{m l} \partial_{n} \partial_{m} \omega_{0}^{a j} \partial_{j} \partial_{k} \partial_{l} \omega_{0}^{b c}\right]+ \text { cycl. }(a b c)=0 .
\end{aligned}
$$

$\omega_{2}^{j k}$ que resolva esta equação tem que ser a soma dos monomiais que contêm três $\omega_{0}$ e quatro derivadas. A forma mais geral é:

$$
\begin{aligned}
\omega_{2}^{b c} & =c_{1} \partial_{m} \omega_{0}^{n l} \partial_{n} \omega_{0}^{m k} \partial_{l} \partial_{k} \omega_{0}^{b c}+c_{2} \partial_{k} \omega_{0}^{b m} \partial_{l} \omega_{0}^{c n} \partial_{n} \partial_{m} \omega_{0}^{k l} \\
& +c_{3} \partial_{n} \partial_{k} \omega_{0}^{b m} \partial_{m} \partial_{l} \omega_{0}^{c n} \omega_{0}^{k l} .
\end{aligned}
$$

Depois dos cálculos complicados achamos que $c_{1}=-\frac{1}{12}, c_{2}=0$ e $c_{3}=\frac{1}{6}$. A mesma solução da equação (6.72) foi obtida em [75]. Existem pelo menos dois casos quando as correções para $\omega$ não são necessárias. Primeiro é a estrutura de Poisson linear, temos $\omega_{2}=0$, como segue de (6.73). O outro caso é o caso bi-dimencional. De fato, a condição de consistência é trivial em duas dimensões, e quaisquer $\omega_{0}$ e $\omega_{2}$ a resolvem. Já que $\omega_{0}$ em duas dimenções não é restrita pela identidade de Jacobi, (ou seja, ela pode ser completamente geral), a escolha mais natural é $\omega_{2}=0$.

Agora podemos construir o operador $\hat{\omega}_{3}^{i j}$ calculando $W\left(\omega_{0}^{i j}+\alpha^{2} \omega_{2}^{i j}\right)$. A forma explícita é:

$$
\begin{aligned}
& \alpha^{-3} \hat{\omega}_{3}^{i j}=\left(\left(\Gamma_{1}^{i j k}+\frac{1}{2} \Gamma_{0}^{j m n} \partial_{m} \partial_{n} \omega_{0}^{i k}\right) \partial_{a} \partial_{b} \omega_{0}^{i j}+\left(\Gamma_{0}^{i j l k}+\frac{2}{3} \Gamma_{0}^{i j m} \partial_{m} \omega_{0}^{l k}+\right.\right. \\
& \left.\quad \frac{1}{3} \omega_{0}^{i m} \partial_{m} \Gamma_{0}^{j l k}+\frac{1}{6} \omega_{0}^{i m} \partial_{m} \omega_{0}^{j n} \partial_{n} \omega_{0}^{l k}+\frac{1}{6} \omega_{0}^{i m} \omega_{0}^{j n} \partial_{m} \partial_{n} \omega_{0}^{l k}\right) \partial_{a} \partial_{b} \partial_{l} \omega_{0}^{i j} \\
& \left.\quad+\omega_{0}^{l k} \partial_{l} \omega_{2}^{i j}+\omega_{2}^{l k} \partial_{l} \omega_{0}^{i j}\right) \partial_{k} \\
& \quad+\left(\Gamma_{1}^{a k l} \partial_{a} \omega_{0}^{i j}+\left(\frac{3}{2} \Gamma_{0}^{a b k l}+\frac{1}{2} \omega_{0}^{a m} \partial_{m} \Gamma_{0}^{b k l}+\Gamma_{0}^{b m k} \partial_{m} \omega_{0}^{a l}\right) \partial_{a} \partial_{b} \omega_{0}^{i j}\right. \\
& \left.\quad+\left(\Gamma_{0}^{a b k} \omega_{0}^{m l}+\frac{1}{2} \omega_{0}^{b n} \partial_{n} \omega_{0}^{a k} \omega_{0}^{m l}\right) \partial_{a} \partial_{b} \partial_{m} \omega_{0}^{i j}\right) \partial_{k} \partial_{l} \\
& \quad+\left(\Gamma_{0}^{b m l} \omega_{0}^{a k} \partial_{a} \partial_{b} \omega_{0}^{i j}+\frac{1}{6} \omega_{0}^{a k} \omega_{0}^{b l} \omega_{0}^{n m} \partial_{a} \partial_{b} \partial_{n} \omega_{0}^{i j}\right. \\
& \left.\quad+\Gamma_{0}^{a k l m} \partial_{a} \omega_{0}^{i j}\right) \partial_{k} \partial_{l} \partial_{m} .
\end{aligned}
$$




\subsubsection{Produto estrela de quarta ordem}

Os termos de ordem $\alpha^{4}$ em $\hat{x}^{i}$ têm a forma

$$
\alpha^{4} \gamma_{4}^{i}=\alpha^{4}\left(\Gamma_{2}^{i j k}(x) \partial_{j} \partial_{k}+\Gamma_{1}^{i j k l}(x) \partial_{j} \partial_{k} \partial_{l}+\Gamma_{0}^{i j k l m}(x) \partial_{j} \partial_{k} \partial_{l} \partial_{m}\right)
$$

onde $\Gamma_{2}^{i j k}(x), \Gamma_{1}^{i j k l}(x)$ e $\Gamma_{0}^{i j k l m}(x)$ têm que ser determinados. A formula (6.27) para $n=3$ leva

$$
\begin{aligned}
\alpha^{-4} G_{4}^{i j k l m}= & 2 \Gamma_{0}^{b m l} \omega_{0}^{a k} \partial_{a} \partial_{b} \omega_{0}^{i j}+\frac{1}{3} \omega_{0}^{a k} \omega_{0}^{b l} \omega_{0}^{n m} \partial_{a} \partial_{b} \partial_{n} \omega_{0}^{i j}+2 \Gamma_{0}^{a k l m} \partial_{a} \omega_{0}^{i j} \\
+ & 3 \Gamma_{0}^{j l m n} \partial_{n} \omega_{0}^{i k}-3 \Gamma_{0}^{i l m n} \partial_{n} \omega_{0}^{j k}-2 \Gamma_{0}^{i m n} \partial_{n} \Gamma_{0}^{j k l}+2 \Gamma_{0}^{j m n} \partial_{n} \Gamma_{0}^{i k l} \\
+ & \omega_{0}^{i n} \partial_{n} \Gamma_{0}^{j k l m}-\omega_{0}^{j n} \partial_{n} \Gamma_{0}^{i k l m}, \\
\alpha^{-4} G_{4}^{i j k l}= & \left(3 \Gamma_{0}^{a b k l}+3 \omega_{0}^{a m} \partial_{m} \Gamma_{0}^{b k l}+2 \Gamma_{0}^{b m k} \partial_{m} \omega_{0}^{a l}\right) \partial_{a} \partial_{b} \omega_{0}^{i j} \\
& +2 \Gamma_{1}^{a k l} \partial_{a} \omega_{0}^{i j}+\left(2 \Gamma_{0}^{a b k} \omega_{0}^{m l}+\omega_{0}^{b n} \partial_{n} \omega_{0}^{a k} \omega_{0}^{m l}\right) \partial_{a} \partial_{b} \partial_{m} \omega_{0}^{i j} \\
& +3 \Gamma_{0}^{j l m n} \partial_{m} \partial_{n} \omega_{0}^{i k}-3 \Gamma_{0}^{i l m n} \partial_{m} \partial_{n} \omega_{0}^{j k}-2 \Gamma_{1}^{i l m} \partial_{m} \omega_{0}^{j k} \\
& +2 \Gamma_{1}^{j l m} \partial_{m} \omega_{0}^{i k}+\omega_{0}^{i n} \partial_{n} \Gamma_{1}^{j k l}-\omega_{0}^{j n} \partial_{n} \Gamma_{1}^{i k l} \\
& -\Gamma_{0}^{i m n} \partial_{m} \partial_{n} \Gamma_{0}^{j k l}+\Gamma_{0}^{j m n} \partial_{m} \partial_{n} \Gamma_{0}^{i k l}, \\
\alpha^{-4} G_{4}^{i j k}= & \left(2 \Gamma_{1}^{i j k}+\Gamma_{0}^{j m n} \partial_{m} \partial_{n} \omega_{0}^{i k}\right) \partial_{a} \partial_{b} \omega_{0}^{i j}+\left(2 \Gamma_{0}^{i j l k}+\frac{4}{3} \Gamma_{0}^{i j m} \partial_{m} \omega_{0}^{l k}\right. \\
+ & \frac{2}{3} \omega_{0}^{i m} \partial_{m} \Gamma_{0}^{j l k}+\frac{1}{3} \omega_{0}^{i m} \partial_{m} \omega_{0}^{j n} \partial_{n} \omega_{0}^{l k} \\
+ & \left.\frac{1}{3} \omega_{0}^{i m} \omega_{0}^{j n} \partial_{m} \partial_{n} \omega_{0}^{l k}\right) \partial_{a} \partial_{b} \partial_{l} \omega_{0}^{i j}+2 \omega_{0}^{l k} \partial_{l} \omega_{2}^{i j}+2 \omega_{2}^{l k} \partial_{l} \omega_{0}^{i j} \\
- & \omega_{0}^{i l} \partial_{l} \omega_{2}^{j k}+\omega_{0}^{j l} \partial_{l} \omega_{2}^{i k}-\omega_{2}^{i l} \partial_{l} \omega_{0}^{j k}+\omega_{2}^{j l} \partial_{l} \omega_{0}^{i k} \\
- & \Gamma_{1}^{i l m} \partial_{l} \partial_{m} \omega_{0}^{j k}+\Gamma_{1}^{j l m} \partial_{l} \partial_{m} \omega_{0}^{i k}-\Gamma_{0}^{i l m n} \partial_{l} \partial_{m} \partial_{n} \omega_{0}^{j k} \\
+ & \Gamma_{0}^{j l m n} \partial_{l} \partial_{m} \partial_{n} \omega_{0}^{i k} \\
&
\end{aligned}
$$

Usando Lema 2, obtemos

$$
\begin{aligned}
& \alpha^{4} \Gamma_{0}^{i j k l m}=\frac{1}{20}\left(G_{4}^{i j k l m}+G_{4}^{i k j l m}+G_{4}^{i l j k m}+G_{4}^{i m j k l}\right) \\
& \alpha^{4} \Gamma_{1}^{i j k l}=\frac{1}{12}\left(G_{4}^{i j k l}+G_{4}^{i k j l}+G_{4}^{i l k j}\right) \\
& \alpha^{4} \Gamma_{2}^{i j k}=\frac{1}{6}\left(G_{4}^{i j k}+G_{4}^{i k j}\right) .
\end{aligned}
$$


Depois com ajuda das fórmulas (6.14), (6.5), (6.38) e (6.85) construímos o produto estrela da quarta ordem. Ele pode ser representado como

$$
f \star_{4} g=\alpha^{4} \sum L_{m, n}(f, g)
$$

onde cada termo na soma tem $m$ derivadas atuando na função $f$ e $n$ derivadas atuando na $g$. O produto estrela da quarta ordem é simétrico. Portanto,

$$
L_{m, n}(f, g)=L_{n, m}(g, f) .
$$

Todos $L_{m, n}(f, g)$ que não são igual a zero com $m \leq n$ são escritos abaixo.

$$
\begin{aligned}
& L_{1,2}(f, g)=\partial_{i} f \Gamma_{2}^{i j k} \partial_{j} \partial_{k} g \\
& L_{1,3}(f, g)=\partial_{i} f \Gamma_{1}^{i j k l} \partial_{j} \partial_{k} \partial_{l} g \\
& L_{1,4}(f, g)=\partial_{i} f \Gamma_{0}^{i j k l m} \partial_{j} \partial_{k} \partial_{l} \partial_{m} g \\
& L_{2,2}(f, g)=\partial_{i} \partial_{m} f\left(\Gamma_{1}^{i j l} \partial_{j} \omega_{0}^{m n}+\frac{3}{2} \Gamma_{0}^{i j k l} \partial_{j} \partial_{k} \omega_{0}^{m n}\right. \\
&\left.+\omega_{0}^{i j} \partial_{j} \Gamma_{1}^{m n l}+\frac{1}{2} \Gamma_{0}^{i j k} \partial_{j} \partial_{k} \Gamma_{0}^{m n l}+\frac{3}{2} \Gamma_{1}^{i m n l}\right) \partial_{l} \partial_{n} g \\
& L_{2,3}(f, g)=\partial_{i} \partial_{m} f\left(\frac{1}{2} \omega_{0}^{i j} \partial_{j} \Gamma_{0}^{m n k l}+\frac{1}{2} \Gamma_{1}^{i l k} \omega_{0}^{m n}\right. \\
&\left.+\frac{3}{2} \Gamma_{0}^{i j k l} \partial_{j} \omega_{0}^{m n}+\Gamma_{0}^{i j k} \partial_{j} \Gamma_{0}^{m n l}+2 \Gamma_{0}^{i j k l m}\right) \partial_{k} \partial_{l} \partial_{n} g \\
& L_{2,4}(f, g)=\partial_{i} \partial_{m} f\left(\omega_{0}^{i j} \Gamma_{0}^{m n k l}+\frac{1}{2} \Gamma_{0}^{i j k l} \Gamma_{0}^{m n l}\right) \partial_{j} \partial_{k} \partial_{l} \partial_{n} g \\
& L_{3,3}(f, g)=\partial_{i} \partial_{j} \partial_{m} f\left(\frac{1}{2} \omega_{0}^{i a} \partial_{a} \omega_{0}^{j k} \Gamma_{0}^{m n l}+\frac{1}{2} \omega_{0}^{i a} \omega_{0}^{j k} \partial_{a} \Gamma_{0}^{m n l}\right. \\
&\left.+\omega_{0}^{i k} \Gamma_{0}^{j a l} \partial_{a} \omega_{0}^{m n}+\frac{2}{3} \Gamma_{0}^{m n l} \Gamma_{0}^{i j k}+\omega_{0}^{m n} \Gamma_{0}^{i j k l}\right) \partial_{k} \partial_{l} \partial_{n} g, \\
& \frac{1}{2} \partial_{i} \partial_{j} \partial_{m} f \omega_{0}^{i a} \omega_{0}^{j k} \Gamma_{0}^{m n l} \partial_{a} \partial_{k} \partial_{l} \partial_{n} g, \\
& L_{3,4}(f, g) \frac{1}{24} \partial_{i} \partial_{j} \partial_{m} \partial_{n} f \omega_{0}^{i k} \omega_{0}^{j l} \omega_{0}^{m a} \omega_{0}^{n b} \partial_{k} \partial_{l} \partial_{a} \partial_{b} g \\
& L_{4,4}(f, g)
\end{aligned}
$$

$\mathrm{Na}$ quarta ordem, bem como em todas as ordens pares,

$$
f \star_{4} g-g \star_{4} f=0,
$$

assim que a condição de consistência é obedecida automaticamente e $\omega$ não precisa ser corregida.

A quinta ordem do produto estrela pode ser calculado diretamente usando o procedimento acima. 


\subsection{Conclusão}

Gostaríamos de notar que em nosso procedimento o cálculo das ordens altas do produto estrela é feito com facilidade e sem muito esforço intelectual, com excessão da solução da condição de consistência para a construção das correções $\omega_{2 n}^{i j}(x)$ para o bi-vetor de Poisson $\omega_{0}^{i j}(x)$, para a qual não pudemos apresentar um algoritmo geral. Em particular, o cálculo do produto estrela de quinta ordem pode ser feito diretamente usando o método proposto e os resultados das ordens anteriores. A condição de consistência na quarta ordem, bem como em todas as ordens pares, é obedecida automaticamente e nenhuma correção para $\omega$ aparece. No entanto, nós não apresentamos aqui as expressões exatas porque elas são extremamente grandes.

As equações para $\omega_{2 n}^{i j}(x)$ que aparecem na nossa abordagem são praticamente identicas às que surgem da abordagem por cohomologias de Hochschild [75]. O estudo das relações entre a nossa abordagem e a abordagem baseada em cohomologias de Hochschild é um problema interessante e nós planejamos estudá-la no futuro. Porém, em alguns casos (por exemplo: caso bi-dimensional ou caso da álgebra de Lie em dimensão arbitrária) a condição de consistência resolve-se automaticamente, e os cálculos do produto estrela podem ser feitos até a ordem arbitrária. Podemos, assim, inclusive tentar deduzir uma expressão exata do produto estrela nestes casos.

\subsection{Algumas fórmulas}

Para o uso da expansão de Duhamel (6.38) temos que calcular os comutadores dos operadores $A=-i p_{m} x^{m}$ e $B=-i p_{j}\left(\hat{x}^{j}-x^{j}\right)$. Denotamos $B_{k}=-i p_{j} \Gamma^{j i_{1} \ldots i_{k}} \partial_{i_{1}} \ldots \partial_{i_{k}}$. Assim

$$
\begin{aligned}
& {\left[B_{k}, A\right]=k\left(-i p_{j}\right)\left(-i p_{i_{1}}\right) \Gamma^{j i_{1} i_{2} \ldots i_{k}} \partial_{i_{2}} \ldots \partial_{i_{k}}, \quad k \geq 2,} \\
& {\left[\left[B_{k}, A\right], A\right]=k(k-1)\left(-i p_{j}\right)\left(-i p_{i_{1}}\right)\left(-i p_{i_{2}}\right) \Gamma^{j i_{1} i_{2} \ldots i_{k}} \partial_{i_{3}} \ldots \partial_{i_{k}}, \quad k \geq 3,} \\
& \quad\left[\left[\left[B_{k}, A\right], A\right], A\right] \\
& \quad=k(k-1)(k-2)\left(-i p_{j}\right)\left(-i p_{i_{1}}\right)\left(-i p_{i_{2}}\right)\left(-i p_{i_{3}}\right) \Gamma^{j i_{1} i_{2} \ldots i_{k}} \partial_{i_{4}} \ldots \partial_{i_{k}}, \quad k \geq 4 .
\end{aligned}
$$




\section{Referências Bibliográficas}

[1] P.A.M. Dirac, Proc.Roy.Soc. A133 (1931) 60.

[2] A. Caldeira and A. Leggett, Physica A121 (1983) 587.

[3] V.G. Kupriyanov, S.L. Lyakhovich and A.A. Sharapov, J.Phys.A38(2005) 8039.

[4] H. von Helmholtz, Journ. f. d. reine u. angew. Math., 100 (1887) 137.

[5] G. Darboux, Lesons sur la Theorie Generale des Surfaces (Gauther-Villars, Paris, 1894).

[6] J. Douglas, Trans. Am. Math. Soc.50, N 71 (1941) 71.

[7] R. Santilli, Ann. Phys. (NY)103 (1977) p.354.

[8] W. Sarlet, J.Math.Phys.19(1978)1049.

[9] V.V. Dodonov, V.I. Man'ko and V.D. Skarzhinsky, Arbitrariness in the choice of action and quantization of the given classical eqations of motion, preprint of P.N. Lebedev Physical Institute (1978).

[10] S.Okubo, Phys.Rev. D22(1980)919.

[11] W. Sarlet, J.Phys.A 15 (1982) 1503.

[12] M. Henneaux, Ann. Phys, 140 (1982) 45.

[13] G. Morandi, C. Ferrario, G. Lo Vecchio, G. Marmo and C. Rubano, Phys. Rep. 188 (1990) 147.

[14] P. Havas, Actra. Phys. Aust. 38 (1973) 145.

[15] S. Hojman and L. Urrutia, J. Math. Phys. 22 (1981) 1896.

[16] S.Hojman and H.Handerston, J.Math.Phys. 22 (1981) 1414.

[17] E.P. Wigner, Phys.Rev. 77 (1950) 11. 
[18] A.P.Balachandran, T.R.Govindrajan and B.Vijayalakshimi, Phys.Rev. D18(1978)1950.

[19] M.Henneaux, L.Sheplley, J.Math.Phys 23 (1982) 2101.

[20] J. Cislo and J. Lopuzanski, J.Math.Phys. 42 (2001) 5163.

[21] P. Tempesta, E. Alfinito, R.Leo and G.Soliani, J.Math.Phys. 43 (2002) 3583.

[22] H. Bateman, Phys.Rev. 38 (1931) 815.

[23] H.Dekker, Phys.Rep. 80 (1981) 1.

[24] E. Celeghini, M. Rasetti and G. Vitello, Ann.Phys. 215 (1992) 156.

[25] R.Banerjee and P.Mukherjee, J.Phys.A35 (2002) 5591.

[26] M. Blasone and P. Jizba, Ann.Phys. 312 (2004) 354.

[27] D. Chruscinski, Ann. Phys. 321 (2006) 840; Ann. Phys. 321 (2006) 854

[28] E. Kanai, Progr.Theor.Phys., 3 (1948) 440.

[29] R.Hasse, J.Math.Phys. 16 (1975) 2005.

[30] D.C.Khandekar and S.V.Lavande, J.Math.Phys. 20 (1979) 1870.

[31] W.E. Brittin, Phys. Rev. 77 (1950) 396; N.A. Lemos, Phys.Rev. D24 (1981) 2338.

[32] I.R. Senitzky, Phys.Rev. 119 (1960) 670.

[33] R.P. Feynman and F.L. Vernon, Ann.Phys. 24 (1963) 118.

[34] H.Haken, Rev.Mod.Phys. 47 (1975) 68.

[35] H.Dekker, Phys.Rev. A16 (1977) 2116.

[36] I.A.Pedrosa and B. Baseia, Phys.Rev. D30 (1984) 765.

[37] D.Walls, G.Miburn, Phys.Rev. A31 (1985) 2403.

[38] H.Grabert, P.Shramm and G. Ingold, Phys.Rep 168 (1988) 115.

[39] Li Hua Yu, Chang-Pu Sun, Phys.Rev. A49 (1994) 592.

[40] I.Joichi, S.Matsumoto and M.Yoshimura, Prog.Theor.Phys., 98 (1997) 9.

[41] M. Rosenau, A. Caldeira, S. Dutra, H. Westfahl, Phys.Rev. A61 (2000) 022107.

[42] A.J. Leggett, J.Phys. (Paris), Colloq. 39, c6-1264 (1978) 
[43] A.O. Caldeira and A.J. Leggett, Phys.Rev.Lett. 46 (1981)211

[44] A.O. Caldeira and A.J. Leggett, Phys. Rev. Lett. 48(1982)1571

[45] A.O. Caldeira and A.J. Leggett, Ann.Phys.149(1983)374.

[46] A. Caldeira and A. Leggett, Phys.Rev. A31 (1985) 1059.

[47] L.D. Landau and E.M. LIfshitz, Mechanics, Oxford:Pergamon, 1962.

[48] A. Peres, Phys.Rev. 167(1968)1449

[49] D.M. Gitman and I.V. Tyutin, Quantization of Fields with Constraints, Springer-Verlag 1990.

[50] N. Seiberg and E. Witten, JHEP 9909 (1999) 032, H.O. Girotti, M. Gomes, V.O. Rivelles, A.J. da Silva, Nucl.Phys. B587 (2000) 299-310, M. Douglas, N. Nekrasov, Rev.Mod.Phys.73 (2001) 977-1029, R. Szabo, Phys.Rept.378 (2003) 207-299.

[51] F. Bayen, M. Flato, C. Fronsdal, A. Lichnerowicz and D. Sternheimer, Annals Phys. 111 (1978) 61.

[52] A. Connes, M. Douglas, A. Schwarz, JHEP 9802:003,1998, M. Douglas, C. Hull, JHEP 9802:008,1998

[53] J.Bellissard, Ordinary quantum Hall effect and non commutative cohomology, Proceedings of the Bad-Schandau Conference on Localization, ed. by W.Weller and P.Ziesche (Tuebner, Leipzig, 1988).

[54] S. Doplisher, L. Fredenhagen and J. Roberts, Commum. Math. Phys. 172 (1995) 187.

[55] A. Smailagic, E. Spalucci, Phys. Rev. D65 (2002) 107701

[56] M. Demetrian, D. Kochan, hep-th/0102050

[57] C. Duval, P. Horvathy, Phys.Lett.B479(2000)284-290

[58] V.P. Nair and A.P. Polychronakos, Phys. Lett. B505 (2001) 267

[59] M. Chaichian, M.M. Sheikh-Jabbari, A. Tureanu, Phys. Rev. Lett. 86 (2001) 2716

[60] M. Chaichian, A. Demichev, P. Presnajder, M.M. Sheikh-Jabbari, A. Tureanu, Phys. Lett. B527 (2002) 149

[61] A.F. Ferrari, M. Gomes, C.A. Stechhahn, Phys.Rev.D76(2007)085008 
[62] J. Gamboa, M. Loewe, J.C. Rojas, Phys. Rev. D64 (2001) 067901

[63] G.Mangano, J. Math. Phys. 39 (1998) 2584

[64] C. Acatrinei, JHEP 09(2001)007.

[65] B. Dragovich, Z. Rakic, Theor. Math. Phys.140 (2004) 1299

[66] A. Smailagic, E. Spalucci, J. Phys. A 36 (2003) 467

[67] H. Tan, J. Phys.A 39 (2006) 15299

[68] F. A. Berezin and M. S. Marinov, Ann. Phys. 104 (1977) 336

[69] G. Dito and D. Sternheimer, 9-54, IRMA Lect. Math. Theor. Phys., 1, de Gruyter, Berlin, 2002. arXiv:math/0201168.

[70] M. Kontsevich, Lett. Math. Phys. 66 (2003) 157 [arXiv:q-alg/9709040].

[71] P.A.M. Dirac, Proc. R. Soc. 167 (1938) 148-169.

[72] L.D. Landau, E.M. Lifshitz, The classical theory of fields, Pergamon, Oxford, 1962.

[73] V.L. Ginzburg, Theoretical Physics and Astrophysics: Aditional Chapters, Moscow: Mir, 1985 (in russian).

[74] E. S. Fradkin and D. M. Gitman, Phys. Rev. D44 (1991)3230

[75] M. Penkava and P. Vanhaecke, Journal of Algebra 227 (2000) 365-393 [arXiv:math.QA/9804022].

[76] E. Poisson, preprint gr-qc/9912045.

[77] F. A. Berezin and M. A. Shubin, "The Schrödinger equation," Kluwer, Dordrecht, 1991.

[78] H.R. Lewis and W.B. Riesenfeld, J.Math.Phys. 10 (1969) 1458.

[79] N.A.Chernikov, The system whose hamiltonian is a time-dependent quadratic form in coordinates and momenta. communications of the Joint Institute for Nuclear Research, Dubna (1990).

[80] J.K. Kim and S.P. Kim, J.Phys.A32 (1999) 2711.

[81] H. Kim and J. Yee, Phys.Rev. A66 (2002) 032117.

[82] Kyu Hwang Yeon, ChungIn UM, T.F.George, Phys.Rev. A68 (2003) 052108.

[83] D.M. Gitman, Nucl. Phys. B468 (1997) 490 
[84] A.A. Deriglazov, Phys.Lett.B555(2003)83-88

[85] J.Schwinger, Phys.Rev. 82 (1951) 664.

[86] R. Brauer and H. Weyl, Amer. Journ. Math. 57 (1935) 425

[87] F.A. Berezin, The Method of Second Quantization (Nauka, Moscow, 1965); Introduction to Algebra and Analysis with Anticommuting Variables (Moscow State University Press, Moscow, 1983); Introduction to Superanalysis (D. Reidel, Dordrecht 1987)

[88] P.A.M.Dirac, Lectures on Quantum Mechanics (Yeshiva University Press, New York 1964)

[89] A. Pinzul, A. Stern, Phys.Lett. B593 (2004) 297-286

[90] W. Behr and A. Sykora, Nucl. Phys. B 698 (2004) 473 [arXiv:hep-th/0309145].

[91] A. Zotov, Mod. Phys. Lett. A 16 (2001) 615 [arXiv:hep-th/0007072].

[92] S. Gutt, Lett. Math. Phys. 7 (1983) 249-258.

[93] G. Dito, Lett. Math. Phys. 48 (1999) 307-322. 